\title{
The role of electronic and vibrational scattering on thermal transport across multiple interfaces in nanostructures
}

\author{
A Dissertation Presented to the \\ Faculty of the School of Engineering and Applied Science \\ University of Virginia \\ In Partial Fulfillment \\ of the Requirements for the Degree of \\ Doctor of Philosophy \\ Mechanical and Aerospace Engineering
}

Ashutosh Giri

November, 2016 
APPROVAL SHEET

The dissertation is submitted in partial fulfillment of the requirements for the degree of

Doctor of Philosophy in Mechanical and Aerospace Engineering

\section{Ashutosh Giri}

This dissertation has been read and approved by the Examining Committee:

Prof. Patrick E. Hopkins, Advisor

Dean Pamela M. Norris, Chairman

Prof. Joeseph Poon

Prof. Stephen J. McDonnell

Dr. Sean W. King

Accepted for the School of Engineering and Applied Science:

Craig H. Benson, Dean

School of Engineering and Applied Science

November, 2016 


\begin{abstract}
Rapid miniaturization and faster working speeds in electronic devices has led to challenges in their thermal mitigation. However, thermal conductivity of the constituent materials in the nano-devices, even though a very critical parameter in their design, is mostly overlooked and the thermal performance of the device is mainly controlled through packaging techniques. However, it would be advantageous for a diverse spectrum of technologies, which ultimately fail due to either high density of interfaces or due to high operating frequencies, to control and tune thermal properties at the submicron length scale and femtoto-picosecond time scales. Therefore, a comprehensive understanding of how heat flows across nanosystems that are mostly limited by interfacial thermal transport would prove to be quintessential for the design of various electronic devices. For example, the contribution of electronic thermal resistance across metal/semiconductor interfaces has been a subject of debate for the past several decades; understanding the intrinsic electron-electron, electron-phonon and electron-boundary scattering mechanisms in these devices could potentially lead to transistors with higher operating frequencies. Moreover, with the advent of new fabrication technologies, the role of phononic-driven thermal resistances across hybrid interfaces of inorganic/organic materials and amorphous semiconductor superlattices (SLs) with high density of interfaces has largely been unexplored.

It is the goal of this work to comprehensively explore interfacial thermal transport in these material systems by understanding the fundamental scattering mechanisms of the energy carriers, which dominate thermal transport at various length and time scales. In this regard, a combination of time-domain thermoreflectance (TDTR) experiments and non-equilibrium molecular dynamics (NEMD) simulations will be implemented to achieve these goals.
\end{abstract}

The nonequilibrium between electrons and phonons at metal/semiconductor interfaces in high frequency microelectronic devices serves as a bottleneck to heat transfer in these 
devices. To understand this phenomena, TDTR is used to probe the highly non-equilibrium condition that is induced due to pulse absorption by thin Au films deposited on various dielectric substrates. For electron temperature excursions of $\leq 3,500 \mathrm{~K}$ in $\mathrm{Au}$, it is shown that while the increase in the excited carrier density increases the e-p coupling in the metal, the bond strength between the metal and the substrate dictates the energy transfer rate across the interface during this highly non-equilibrium time regime. Furthermore, at time scales when the electrons have fully thermalized with the lattice, electron-phonon coupling does not influence the phonon-driven thermal boundary conductance.

Another phenomena that lead to device failure are the phonon-driven thermal boundary resistance in multilayered semiconductors used in technologies such as thermoelectric power generation or phase change memory devices. TDTR is used to simultaneously measure the thermal conductivities and heat capacities of hybrid inorganic/organic superlattices and amorphous superlattices. Furthermore, atomistic simulations are implemented to shed light on the intrinsic scattering mechanisms dictating heat flow in these material systems. The goal of these studies is to demonstrate that through judicious choice of individual period thicknesses and the understanding of interfacial thermal resistances in these multilayers, new strategies can be developed to engineer material systems with user defined thermal transport. 


\section{Acknowledgements}

First and foremost, I would like to thank my family for the unconditional love and support that they have shown me throughout these years as an undergraduate and a graduate student. My journey towards getting a doctorate degree would not have been a successful one if my parents did not place my sisters and my own schooling above everything else while facing the hardships involved in providing a world class education for us in a third world country like Nepal. To my mother, who has made a name for herself as an educator and a professor in a patriarchal education system - you continue to be an inspiration for all of my academic endeavors. To my father, who, despite being born in one of the most rural villages in Nepal, has worked his way into providing a good life for his family - your teachings and guidance has always propelled me to reach for the moon while my feet are firmly on the ground. To my sister, who has always pushed me to show my creative side I appreciate our small talks and conversations that we have every now and then. They have helped me cope through these hectic years as a PhD student.

Second, I place my gratitude to one of the leading researchers in the field of Science and engineering today, my advisor Professor Patrick Hopkins who has shown me that being a student of science can be one of the most rewarding experiences. I thank you for the countless hours spent discussing (and g-chatting till 2 am in the mornings) about electrons and phonons and I am sure you will continue to shape the minds of future graduate students and share your love and enthusiasm for science in the coming years as a Professor.

I would also like to take this opportunity to thank my committee members, Dean Pamela Norris, Professor Joseph Poon, Professor Stephen McDonell, and Dr. Sean King for agreeing to serve on my committee. To Dr. Sean King, I am greatly indebted for your continued efforts to provide me with whatever samples I have needed to complete my $\mathrm{PhD}$. I would also like to thank the members of the Exsite lab for sharing this social and academic journey with me.

Last but not the least, I would like to thank my soon to be wife Sneha Pokharel for being my support system throughout these years. The success of this doctoral degree is 
as much mine as it is yours since I cannot conceive of ever being so productive without you being by my side. You have held my hand through tough times and sleepless nights (especially the 2015 earthquake in Nepal, which I would have struggled to get through if I did not have your support). Throughout this journey, you have taught me the real meaning of compassion; you are the yin to my yang. As I gear up for my post $\mathrm{PhD}$ life, I am glad that I will be sharing the rest of my journey with you. 


\section{Contents}

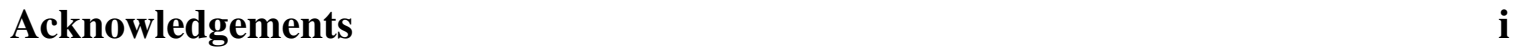

$\begin{array}{lll}1 & \text { Introduction } & 1\end{array}$

1.1 Motivation and background $\ldots \ldots \ldots \ldots$. . . . . . . . . . . . . . . . . . .

1.2 Outline and objectives $\ldots \ldots \ldots \ldots$. . . . . . . . . . . 7

2 Interfacial transport: theory and experiment 10

2.1 Interfacial thermal transport $\ldots \ldots \ldots \ldots$

2.2 Diffuse mismatch model for electron energy exchange at a metal/nonmetal

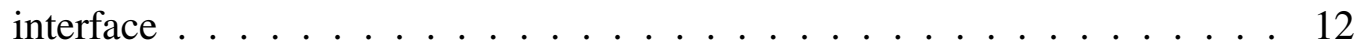

2.3 Molecular dynamics simulations . . . . . . . . . . . . . . . . 18

2.4 NEMD simulations on LJ-based solid/solid interfaces . . . . . . . . . . . . 19

2.4 .1 Computational domain setup . . . . . . . . . . . . . . . 19

2.4 .2 Density of states calculations and modal analysis . . . . . . . . . 21

2.4 .3 Spectral contributions to thermal boundary conductance . . . . . . 24

2.5 Time domain thermoreflectance (TDTR) . . . . . . . . . . . 25

$2.5 .1 \quad$ Experimental setup . . . . . . . . . . . . . 26

2.5 .2 Thermal model and data analysis . . . . . . . . . . . . . . . . . 29

2.5 .3 The two-temperature model . . . . . . . . . . . . . . 33

3 Mechanisms of electron-phonon coupling at interfaces 38

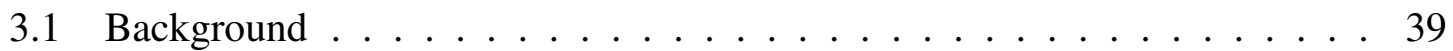

3.2 Experimental considerations . . . . . . . . . . . . . . . . 40

3.3 Ballistic transport and electron scattering at the interface . . . . . . . . . 43

3.4 Thermal diffusion processes after electron-phonon equilibration . . . . . . 50

3.5 Summary $\ldots \ldots \ldots \ldots \ldots \ldots \ldots \ldots$

4 Thermal transport in hybrid superlattices $\quad 56$

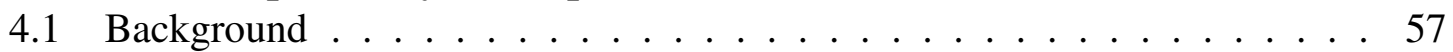

4.2 Experimental details . . . . . . . . . . . . . . . . . . 59

$4.2 .1 \quad$ Sample fabrication and characterization . . . . . . . . . . . . 59

$4.2 .2 \quad$ TDTR measurements and analyses . . . . . . . . . . . . . . 62

4.3 Thermal conductivity measurements for the $\mathrm{TiO}_{2}$-based SLs . . . . . . . . 66

4.4 Thermal conductivity measurements for the ZnO-based SLs . . . . . . . . 68

4.5 Thermal boundary conductance in organic/inorganic composites . . . . . 73 
4.6 Effect of the type and thickness of the organic layer on thermal transport . . 80

4.7 Heat capacity measurements $\ldots \ldots \ldots \ldots$. . . . . . . . . . . . . . . . . . . . 84

4.8 Summary $\ldots \ldots \ldots \ldots \ldots \ldots \ldots$

5 Thermal transport across amorphous-based confined thin films and superlat$\begin{array}{ll}\text { tices } & 89\end{array}$

5.1 Introduction . . . . . . . . . . . . . . . . . . . 90

5.2 Disordered interfacial thin films . . . . . . . . . . . . . . . . 93

5.2 .1 Computational details . . . . . . . . . . . . 93

5.2 .2 Thermal boundary resistance across interfacial thin films . . . . . . 95

5.3 Thermal transport across amorphous/amorphous superlattices . . . . . . . . 100

5.3 .1 Computational details . . . . . . . . . . . . . 100

5.3.2 Thermal boundary resistance and thermal conductivity of amorphous Stillinger Weber-based Si/Ge superlattices . . . . . . . . . 101

5.3.3 Experimental investigation on the thermal properties of amorphous superlattices . . . . . . . . . . . . . . 105

5.3.4 Thermal boundary resistance and thermal conductivity of amorphous SiC:H/SiOC:H superlattices . . . . . . . . . . . . . 108

5.4 Thermal transport across amorphous/crystalline superlattices . . . . . . . 109

5.4 .1 Computational details . . . . . . . . . . . . . . 109

5.4.2 Thermal conductivity of amorphous/crystalline Si/Ge superlattices . 111

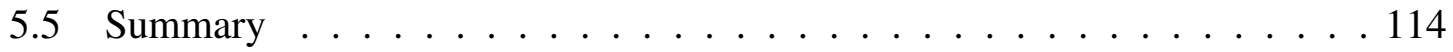

6 Conclusions and future work $\quad 116$

6.1 Summary of major results . . . . . . . . . . . . . . . . 116

6.2 Future work . . . . . . . . . . . . . . . . . . . 118 


\section{List of Figures}

1.1 Thermal boundary conductance as a function of temperature for various solid/solid interfaces (Pd/Ir [14], Al/Cu [15], TiN/MgO [16], Al/Si [17], $\mathrm{Au} / \mathrm{SAM} / \mathrm{Au}$ [12], Pb/diamond [18], Bi/diamond [19] and Ag/CuPc [20]).] . 3

1.2 Illustrations of (a) hybrid superlattices with periodic inclusion of hydroquinone molecules inbetween $\mathrm{ZnO}$ inorganic constituents, and (b) amorphous superlattices with period length $L$ comprised of alternating layers of inorganic constituents. ................ 6

2.1 Schematic diagram showing the transitions in the three energy levels in the metal and phonon frequencies in the non-metal. . . . . . . . . . . . . 14

$2.2 h_{\mathrm{es}}$ predicted from the theoretical framework developed in this work compared to the experimentally determined $h_{\mathrm{es}}$ from the TTR data analyzed with the two temperature model [36]. The $h_{\mathrm{es}}$ increases linearly with increasing effective electron temperature. . . . . . . . . . . . . . 17

2.3 (Top panel) Atomistic illustration of the computational domain between LJ-based solid/solid system. (Bottom panel)Temperature profile after a steady heat flux is imposed. . . . . . . . . . . . . . . . . . 20

2.4 Normalized thermal conductivity accumulation predicted from the NEMD calculations for a homogeneous LJ argon with an imaginary interface in the middle of the computational domain (at $50 \mathrm{~K}$ ) as a function of $\omega_{\mathrm{Ar}} / \omega_{\mathrm{Ar} \text { max }}$. For comparison, the result from Ref. [75] that is based on the Boltzmann transport equation in conjunction with anharmonic lattice dynamics calculation performed at $50 \mathrm{~K}$ are also shown. . . . . . . . . . . . . 23

2.5 (a) Normalized thermal boundary accumulation at the interface of a two LJ-based solids at $1 \mathrm{~K}$ and $30 \mathrm{~K}$ with $\varepsilon_{\mathrm{ss}}=0.0103 \mathrm{eV}$. Also included is the prediction for a weak cross-species interaction strength across the interface with $\varepsilon_{\mathrm{ss}}=0.0026 \mathrm{eV}$ at $30 \mathrm{~K}$. Also included are the DOS (a.u.) of the bulk solids. (b) NEMD-predicted thermal boundary conductances across the two LJ-based solids differentiated by mass as a function of temperature for strong and weak cross-species interaction. . . . . . . . . . . . . . . 24

2.6 Schematic of the two-color TDTR setup . . . . . . . . . . . . . . 27

2.7 Sensitivities of the in-phase signal (for an ALD grown $\mathrm{ZnO}$ thin film) to the thermal boundary conductances at $\mathrm{Al} / \mathrm{ZnO}$ and $\mathrm{ZnO} / \mathrm{Al}_{2} \mathrm{O}_{3}$ interfaces and thermal conductivities of $\mathrm{Al}, \mathrm{ZnO}$ and $\mathrm{Al}_{2} \mathrm{O}_{3}$. . . . . . . . . . . . 30 
2.8 Sensitivity contour plots showing the interrelationship between (a) $h_{\mathrm{K}}$ at $\mathrm{ZnO} / \mathrm{Al}_{2} \mathrm{O}_{3}$ interface and $h_{\mathrm{K}}$ at $\mathrm{Al} / \mathrm{ZnO}$ interface, (b) $h_{\mathrm{K}}$ at Al/ZnO interface and $\kappa_{\mathrm{ZnO}}$ and (c) $h_{\mathrm{K}}$ at $\mathrm{ZnO} / \mathrm{Al}_{2} \mathrm{O}_{3}$ and $\kappa_{\mathrm{ZnO}}$ in the three-layer thermal model for the purely ALD grown $\mathrm{ZnO}$ thin film at $8.8 \mathrm{MHz}$ pump modulation frequency and room temperature. . . . . . . . . . . . 31

2.9 TDTR signal on $26 \mathrm{~nm} \mathrm{Au} / \mathrm{Si}$ sample at an absorbed laser fluence of $\mathrm{F}$ $=3.52 \mathrm{~J} \mathrm{~m}^{-2}$ and corresponding best fit using the modified TTM with a nonlinear thermoreflectance model. (inset) Picosecond acoustic response of the film used to measure the thickness. . . . . . . . . . . . . . . . 36

3.1 TDTR data on $\mathrm{Au} /$ fused silica (red square) and $\mathrm{Au} / \mathrm{Ti} /$ fused silica (blue circle) samples at room temperature and corresponding best-fits using the TTM with a nonlinear thermoreflectance model. The data is normalized by the maximum magnitude of the signal from the lock-in amplifier. . . . . . 43

$3.2 G_{\text {eff }}$ as a function of maximum effective electron temperature plus maximum phonon temperature from DC laser heating for $\mathrm{Au} / \mathrm{Ti} / \mathrm{fused}$ silica (blue circle), $\mathrm{Au} / \mathrm{Ti} / \mathrm{Si}$ (red square) and $\mathrm{Au} / \mathrm{Ti} / \mathrm{sapphire} \mathrm{(black} \mathrm{triangle).} \mathrm{For}$

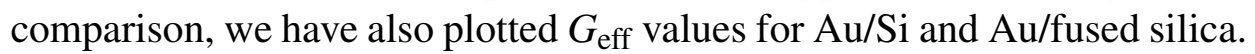
The hollow symbols represent data for Au films with a thickness of $40 \mathrm{~nm}$ with Ti adhesion layer on different substrates. After accounting for $\Delta T_{\mathrm{DC}}$ in $T_{\mathrm{p}}{ }^{\max }$ (due to local heating of the Au lattice), TTM fits to the Au/fused silica data results in similar temperature trends between the determined $G_{\text {eff }}$ for $\mathrm{Au} /$ fused silica and $\mathrm{Au} / \mathrm{Si}$ systems. However, we observed a much larger enhancement in $G_{\text {eff }}$ for systems with the inclusion of the Ti layer. . . 44

3.3 TDTR data on $20 \mathrm{~nm} \mathrm{Au} / \mathrm{Pt} / \mathrm{Si}$ and $40 \mathrm{~nm} \mathrm{Au} / \mathrm{Pt} / \mathrm{Si}$ samples with the corresponding fits using the TTM with a nonlinear thermoreflectance model for the initial few picoseconds. The thermoreflectance responses at longer pump-probe delay times are also shown where the periodic oscillations in the data for 10-100 $\mathrm{ps}$ is representative of strain wave propagation. . . . . . 47

$3.4 h_{\mathrm{es}}$ as a function of effective electron temperature for three different substrates at $T_{p}=300 \mathrm{~K}$. The closed symbols represent calculations with parameters for $\mathrm{Au}$ electrons whereas the hollow symbols represent calculations with parameters for Ti electrons. The values of the predicted $h_{\mathrm{es}}$ are different for different phonon dispersions of the substrate and are in good qualitative agreement with the conductances measured in Fig. 3.2 for the

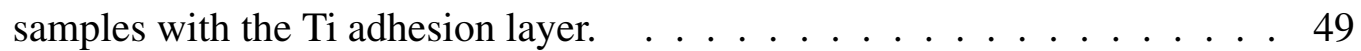

3.5 TDTR data for the initial $1000 \mathrm{ps}$ after laser pulse absorption for (a) 40 $\mathrm{nm} \mathrm{Au} / \mathrm{Ti} / \mathrm{Al}_{2} \mathrm{O}_{3}$ and (b) $40 \mathrm{~nm} \mathrm{Au} / \mathrm{Ti} / \mathrm{Si}$ and (c) $40 \mathrm{~nm} \mathrm{Au} / \mathrm{Ti} / \mathrm{SiO}_{2}$. The TDTR signal decays rapidly for the first few picoseconds and is related to the electron-phonon coupling process in the thin films. For the 10 to $100 \mathrm{ps}$ time range, samples with the Ti layer show a slow rise in the signal which is attributed to the heating of the Au layer due to heat flow from the Ti layer underneath. This is in contrast to the decreasing TDTR signal (and oscillating picosecond acoustic signal) shown in Fig. 2.6 for Au deposited directly on Si without a Ti adhesion layer. . . . . . . . . . . . . . . . 51 
4.1 Characteristic grazing incidence X-ray diffraction (GIXRD) patterns for the control samples and hybrid SLs with varying ALD/MLD ratios for (a) $\mathrm{TiO}_{2}$-based films and (b) $\mathrm{ZnO}_{2}$-based films. . . . . . . . . . . . . . . . 59

4.2 Characteristic XRR patterns for the (a) $\mathrm{TiO}_{2}$ - and (b) $\mathrm{ZnO}$ - based films. . . 61

4.3 Sensitivities of ratio $\left(-V_{\text {in }} / V_{\text {out }}\right)$ to the thermophysical properties of the $(\mathrm{ZnO})_{\mathrm{x}=7 \mathrm{~nm}} / \mathrm{HQ}$ sample as a function of pump-probe time delay at $8.8 \mathrm{MHz}$ pump modulation frequency. . . . . . . . . . . . . . 62

$4.4 \quad$ Sample data and best-fit curves for the $(\mathrm{ZnO})_{\mathrm{x}=7.0 \mathrm{~nm}} / \mathrm{HQ}$ (red squares) and $(\mathrm{ZnO})_{\mathrm{x}=7.0 \mathrm{~nm}} / \mathrm{HQ}$ (black circles) along with uncertainties (dotted lines) at

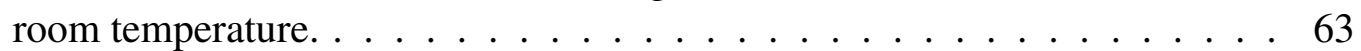

4.5 Sensitivities of ratio $\left(-V_{\text {in }} / V_{\text {out }}\right)$ to the thermo-physical properties of the As-Dep $\left(\mathrm{TiO}_{2}\right)_{\mathrm{x}=0.5 \mathrm{~nm}} / \mathrm{HQ}$ sample as a function of pump-probe time delay at (a) $8.8 \mathrm{MHz}$ pump modulation frequency and (b) $3.72 \mathrm{MHz}$ pump modulation frequency. (c) Sensitivity contour plot showing the interrelationship between thermal conductivity and heat capacity of the As-Dep $\left(\mathrm{TiO}_{2}\right)_{\mathrm{x}=0.5 \mathrm{~nm}} / \mathrm{HQ}$ sample at $3.72 \mathrm{MHz}$ and $8.8 \mathrm{MHz}$ pump modulation frequencies. ....................... 64

4.6 Thermal conductivities of as-deposited and annealed $\mathrm{TiO}_{2}$-based $m=40$ SLs plotted as a function of temperature. For comparison, the measured thermal conductivities of an ALD grown amorphous $\mathrm{TiO}_{2}$ film along with the thermal conductivities of bulk, single crystal $\mathrm{TiO}_{2}$ (Ref. [151]), a polycrystalline sputtered film with $17 \mathrm{~nm}$ grain size (Ref. [150]) and anatase thin film (Ref. [149]) are also shown. The calculated minimum in thermal conductivity for $\mathrm{TiO}_{2}$ is also shown for comparison. . . . . . . . . . . . 67

4.7 Measured thermal conductivities as a function of temperature for $(\mathrm{ZnO})_{\mathrm{X}} / \mathrm{HQ}$ SLs with varying $x$. The error bars include uncertainties due to repeatability, Al thickness measurement and uncertainties in the parameters used in the thermal model. Also plotted are the thermal conductivities for a 180 nm ALD-grown homogenous ZnO film taken from Ref. [91]. . . . . . . . . 68

4.8 Comparison of the experimentally measured heat capacity in Ref. [153] (hollow circles) to the values predicted by Eq. 4.3 (solid line). . . . . . . . 70

4.9 Thermal conductivity as a function of the inorganic layer thickness for SLs with single HQ layers at $78 \mathrm{~K}$ and $300 \mathrm{~K}$. Along with that, the predicted thermal conductivities as a function of SL period thickness calculated from Eq. 4.4 at $78 \mathrm{~K}$ and $300 \mathrm{~K}$ are also shown. The effective thermal conductivity model assuming only phonon-boundary scattering at the HQ-layer interface described in Eq. 4.4 provides reasonable agreement with the measured thermal conductivities for these SLs. (inset) The thermal conductivity for these hybrid SLs is inversely proportional to the $\mathrm{ZnO} / \mathrm{HQ} / \mathrm{ZnO}$ interface

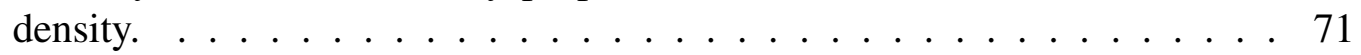


4.10 (a) The mean thermal boundary conductances of interfaces in $\mathrm{ZnO}_{\mathrm{x}} / \mathrm{HQ}$ SLs derived from thermal conductivity measurements shown in Fig. 4.7 , Also plotted are the mean conductances of interfaces in W/Al ${ }_{2} \mathrm{O}_{3}$ SLs [158] and AlN/GaN SLs [4] for comparison. (b) The mean thermal boundary conductances derived for $\mathrm{x}=7.0 \mathrm{~nm}$ and $13.1 \mathrm{~nm}$ as a function of temperature for the $\mathrm{ZnO}$ based SLs. The calculations of maximum conductance in $\mathrm{ZnO}$ with phonon transmission coefficient equal to unity are also shown. Also included for comparison is the calculation of the DMM for a $\mathrm{ZnO} / \mathrm{ZnO}$ interface (i.e., $50 \%$ transmission of the $\mathrm{ZnO}$ phonon flux). . . . . 74

4.11 Spectral heat flux in $\mathrm{ZnO}$ as a function of the wave-vector in the $\Gamma \rightarrow \mathrm{M}$

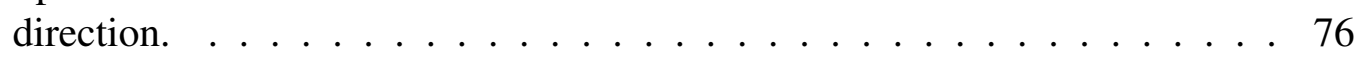

4.12 Schematic of the MD computational domain with 1400 atoms representing the HQ monolayer interspersed between the inorganic layers. The green \begin{tabular}{ll}
\hline atoms are carbon, yellow atoms are oxygen and the red atoms are hydrogen. & 78
\end{tabular}

4.13 Comparison of vibrational density of states for a HQ layer (obtained via molecular dynamics) and $\mathrm{ZnO}$ (obtained from phonon dispersion in Ref. [154]). (inset) The complete local phonon density of states for the HQ layer showing the discrete spikes at a range of phonon frequencies. . . . . . . . . 79

4.14 Top panel depicts unit cells with increasing number of hydroquinone molecules. (a) Thermal conductivity measurements at room temperature as a function of the number of MLD cycles performed. Calculation of Eq. 4.4 for the inorganic layer thickness is also shown for comparison. The measured thermal conductivity for the SLs deviates from the prediction of Eq. 4.4 as the HQ layer thicknesses increase. (b) Effective resistances of inorganic/organic/inorganic interfaces with varying number of hydroquinone layers derived from the thermal conductivities shown in (a). . . . . . . . . . 81

4.15 Thermal conductivities of $\mathrm{ZnO}$-and $\mathrm{TiO}_{2}$-based SLs as a function of SL period thickness. The measured thermal conductivity for a $k: m=1: 1$ hybrid film reported in Ref. [44] is also plotted (hollow triangle). The calculated minimum in thermal conductivities for $\mathrm{ZnO}$ and $\mathrm{TiO}_{2}$ are also shown for comparison. Note, for the case of as-deposited and annealed $\mathrm{TiO}_{2}$, the SL period of $\sim 100 \mathrm{~nm}$ are for the corresponding purely ALD grown samples with $k=0$ (hollow symbols). . . . . . . . . . . . . . 83

4.16 Measured volumetric heat capacities as a function of temperature for (a)

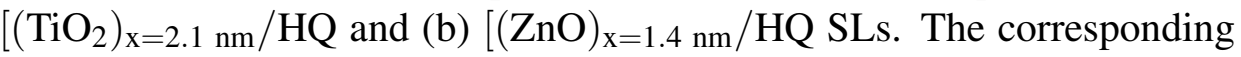
bulk heat capacities for the inorganic constituents are also shown for comparison [168, 169]. . . . . . . . . . . . . . . . . . . . . . 85

4.17 (a) Volumetric heat capacities of the $\mathrm{TiO}_{2}$ - and $\mathrm{ZnO}$-based thin films plotted as a function of density along with the values of bulk heat capacities for the corresponding inorganic constituents. (b) Specific heat capacities plotted as a function of density. In these plots, the data are designated as follows: $\mathrm{TiO}_{2}$ (circles), $\mathrm{ZnO}$ (triangles), corresponding bulk (open symbol), ALD/MLD SLs (closed symbols). For comparison, the heat capacity measured by Liu et al. [44] for a $k: m=1: 1 \mathrm{ZnO}$-based hybrid film is also plotted in the figures. . . . . . . . . . . . . . 86 
5.1 (Top panel) Schematic of the simulation cell for thin films confined between crystalline leads. Thermal flux is applied across the simulation cell in the $z$-direction. (Bottom panel) Temperature profile of $\mathrm{Si} / \mathrm{Ge} / \mathrm{Si}$ systems with disordered and crystalline Ge films. . . . . . . . . . . . . . . . . . 94

5.2 Thermal boundary resistances across the confined thin films predicted via NEMD simulations performed at $500 \mathrm{~K}$ temperature. The TBRs across $\mathrm{Ge} / \mathrm{c}-\mathrm{Si} / \mathrm{Ge}$ are represented as hollow square symbols, solid square symbols represent Ge/a-Si/Ge, hollow green triangles represent $\mathrm{Si} / \mathrm{c}-\mathrm{Ge} / \mathrm{Si}$ and solid green triangles represent $\mathrm{Si} / \mathrm{a}-\mathrm{Ge} / \mathrm{Si}$. For the amorphous thin films, the TBRs for interfacial layers with twice the mass of the layers are also shown. 95

5.3 (a) TBR of Ge/a-Si/Ge structures with $L_{\mathrm{f}}=3 \mathrm{~nm}$ as a function of massmismatch between the leads and the film. (b) Vibrational density of states of crystalline Si and Ge (shaded region), and Si structures with different mass ratios compared to the mass of the Ge lead. . . . . . . . . . . . . . . 99

5.4 Thermal conductivities of amorphous Si/Ge superlattices plotted as a function of (a) period length and (b) interface density. Hollow symbols are data from simulations with domains $125 \AA$ long, and solid symbols are data from simulations with domains $250 \AA$ long. The overlap of hollow and solid symbols indicates size effects did not distort the results. Also plotted is the thermal conductivity predicted by Eq. 5.1 $\mid$ when interfaces are ignored (dashed line), as well as with the best-fit value of $R_{\mathrm{i}}=0.52 \mathrm{~m}^{2} \mathrm{~K} \mathrm{GW}^{-1}$ (solid line). These results are for Si/Ge SLs in which the layers are defined by the same interaction parameters in the potential but differ in their mass

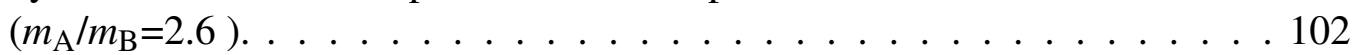

5.5 Sensitivities of ratio $\left(-V_{\text {in }} / V_{\text {out }}\right)$ to the thermophysical properties of the 88 $\mathrm{nm}$ thick SiC:H sample as a function of pump-probe time delay at (a) 2 MHz pump modulation frequency and (b) $10 \mathrm{MHz}$ pump modulation frequency. (c) Sensitivity contour plot showing the interrelationship between thermal conductivity and heat capacity of the $88 \mathrm{~nm} \mathrm{SiC:H}$ sample at 2 $\mathrm{MHz}$ and $10 \mathrm{MHz}$ pump modulation frequencies. . . . . . . . . . . . . 106

5.6 Thermal conductivity of amorphous $\mathrm{SiC}: \mathrm{H}$ and $\mathrm{SiOC}: \mathrm{H}$ films as a function of film thickness. The lack of size effects for both amorphous structures suggest that thermal transport is mostly mediated by diffusons. Also included are the measured thermal conductivities of amorphous $\mathrm{Si}$ from Ref. [212], which shows considerable size effects due to the contribution from propagating modes to the thermal conductivity. . . . . . . . . . . 107

5.7 Thermal conductivities of amorphous $\mathrm{Si} / \mathrm{Ge}$ superlattices plotted as a function of (a) period length and (b) interface density. The hollow symbols are for structures with similar period thicknesses and larger total film thicknesses compared to the ones with the solid symbols. . . . . . . . . . . . . 108

5.8 (Top panel) Schematic of a $27 \times 27 \times 212 \AA^{3}$ simulation cell with $N=0.57$ $\mathrm{nm}^{-1}$ for an amorphous/crystalline superlattice. (Bottom panel) Temperature gradient induced due to the applied flux across the computational domain. (Inset) Local temperature profiles for the amorphous/crystalline layers are similar because of high interface densities. . . . . . . . . . . 110 
5.9 Thermal conductivities of Si/Ge SLs as a function of interface density $N$. Solid square symbols are MD predictions on a-Si/c-Ge SLs with domain lengths of $21 \mathrm{~nm}$ while hollow square symbols are predictions for a-Si/cGe SLs with domain length of $43 \mathrm{~nm}$. For comparison, MD predictions taken from Ref. [218] on crystalline Si/Ge superlattices with two domain lengths are also plotted. . . . . . . . . . . . . . . . . . 111

5.10 (a) Thermal conductivity of a-Si/c-Ge SLs with $N=0.57 \mathrm{~nm}^{-1}$ plotted as a function of mass-mismatch between the layers. (b) Thermal conductivity of amorphous/crystalline Si/Ge SLs described with the Tersoff potential (layers differentiated by mass and bond). . . . . . . . . . . . . . . 113 


\section{List of Tables}

3.1 Parameters used in the thermoreflectance model for the $\mathrm{Au} / \mathrm{Ti} / \mathrm{Si}, \mathrm{Au} / \mathrm{Ti} / \mathrm{Al}_{2} \mathrm{O}_{3}$ and $\mathrm{Au} / \mathrm{Ti} / \mathrm{SiO}_{2}$ structures. $\gamma_{e}$ is the electronic heat capacity coefficient in the TTM. The thicknesses of Au and Ti bi-layers were measured by X-ray reflectivity measurements. . . . . . . . . . . . . 44

4.1 Thicknesses and densities of the thin films measured via XRR. . . . . . . . 62

4.2 Thermal conductivity values measured for the $(\mathrm{ZnO})_{\mathrm{X}} / \mathrm{TOCN}$ hybrids films. 82 


\section{Chapter 1}

\section{Introduction}

Although the study of heat transfer at the microscopic level started a century ago with the pioneering work from Einstein [1] with further refinements by Debye [2], only since the 1980s have the new developments in nanoscale heat transfer lead to the burgeoning of this research field. One of the most pertinent advances in the field has been the realization that Fourier's theory of heat conduction $(Q=-\kappa \nabla T$, where $Q$ is the heat flux, $\kappa$ is the thermal conductivity and $\nabla T$ is the temperature gradient) cannot accurately predict thermal transport when the characteristic length scales are shorter than the mean-free-path of the energy carriers. This has lead to tremendous efforts on solving the full Boltzmann transport equation (BTE) and implementation of rigorous atomic-level modeling techniques such as molecular dynamics (MD) and lattice dynamics (LD) simulations to describe experimental results for systems with reduced dimensionalities (e.g. 2D thin films and 3D nanowires) that are being increasingly used in microelectronic devices.

As the nanoscale size effects pose a major problem in understanding energy transport across materials in device driven applications, the key challenge in mitigating thermal issues almost always relies on accurately predicting interfacial transport across several materials that make up the device. In fact, over the past few decades, interfaces have been shown to limit thermal transport in nanosystems, as varied as superlattices (SLs) [3-5] and semiconductor-based microelectronic devices [6-8] to organic-based nanocomposites [9- 
$12]$.

Unlike in bulk crystals, thermal transport across interfaces depends not only on the intrinsic properties of the materials comprising the interface, but also on the extrinsic properties such as surface chemistry, interfacial non-idealities and strength of bonding between the materials [13]. Along with these considerations, correctly predicting interfacial thermal transport requires knowledge of contributions at different time-scales from the fundamental energy carriers that have spectral distributions in their densities and mean free paths. Even though the growing interest in the field of nanoscale heat transfer has lead to a better understanding of these physical phenomena for several material systems, there are still debates over the accurate interpretation of some experimental results and lack of experimental data for others, which needs to be addressed. This chapter will discuss some of these prior works and motivating factors that lead to the conceptualization of the works presented in this thesis. Following these motivations, the rest of the dissertation will be outlined.

\subsection{Motivation and background}

The first quantitative measurement of thermal boundary conductance (TBC), $h_{\mathrm{K}}$, was reported between $\mathrm{Cu}$ and liquid $\mathrm{He}$ by Kapitza in 1941 [21]. Kapitza [21] quantified $h_{\mathrm{K}}$ as the ratio of the heat flux across the interfacial region to the temperature drop $\Delta T$. Since the scattering mechanisms of the fundamental energy carriers (i.e., electrons and phonons) at the interface influence the $h_{\mathrm{K}}[13]$, advancing the theoretical understanding of these scattering processes is of utmost importance to advancing nanotechnology.

Electrons are the primary heat carriers in metals and phonons dominate the transport process in insulators and semiconductors, and previous theories and experiments have alluded to various electron and/or phonon scattering processes that drive $h_{\mathrm{K}}$ across interfaces composed of different types of materials [7, 13, 22, 24]. Figure. 1.1, shows experimentally measured $h_{\mathrm{K}}$ for various interfaces in which either electrons or phonons are the primary energy carriers driving $h_{\mathrm{K}}$. For example, the heat transport pathway between two metals 


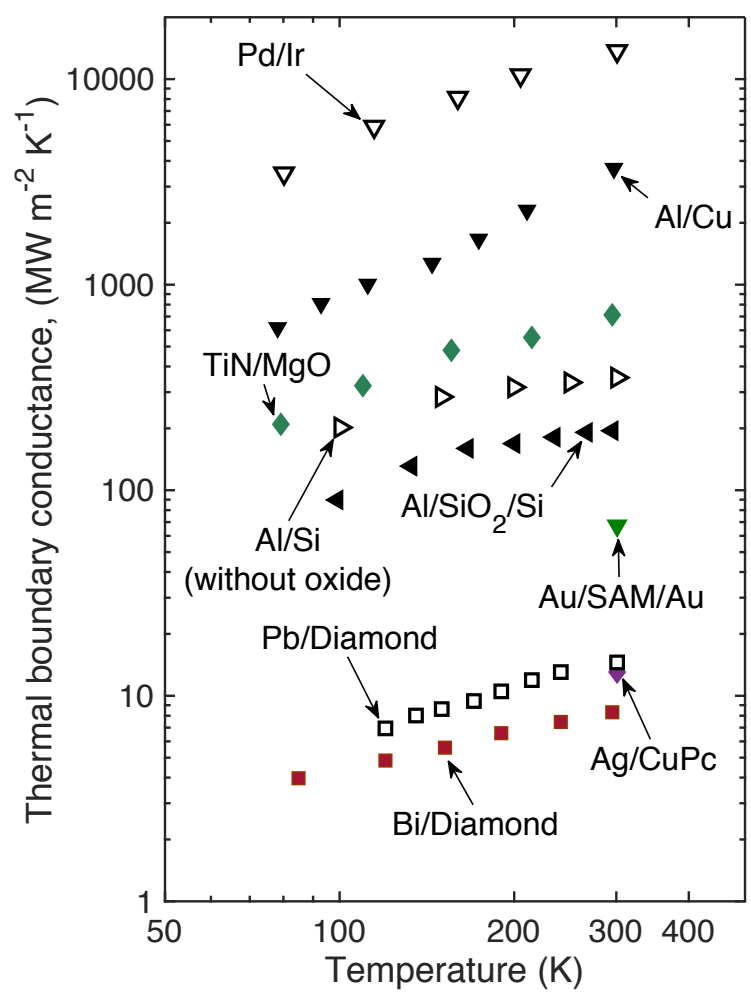

Figure 1.1: Thermal boundary conductance as a function of temperature for various solid/solid interfaces (Pd/Ir [14], Al/Cu [15], TiN/MgO [16], Al/Si [17], Au/SAM/Au [12], $\mathrm{Pb} /$ diamond [18], Bi/diamond [19] and $\mathrm{Ag} / \mathrm{CuPc}[20])$.

is dominated by the scattering of electrons that transport energy through the interface as shown for $\mathrm{Al} / \mathrm{Cu}[15]$ and $\mathrm{Pd} / \mathrm{Ir}$ [14] interfaces. As such, these interfaces possess some of the highest measured TBCs. However, at interfaces composed of semiconductors or insulators, electrons cannot physically transmit energy across the interfaces, (assuming large barrier heights and no tunneling) and therefore poses lower TBCs as compared to the metal/metal interfaces. Even for these types of interfaces where the TBC is mainly driven by phonon-phonon (p-p) scattering processes, the measured values of $h_{\mathrm{K}}$ span over two orders of magnitude. The highly acoustically mismatched interfaces ( $\mathrm{Pb} /$ diamond [18] and $\mathrm{Bi} /$ diamond [19]) possess very low TBCs, whereas the interface with the highly matched and epitaxially grown $\mathrm{TiN}$ on $\mathrm{MgO}$ substrate demonstrates the highest measured $\mathrm{TBC}$ for a solid/solid interface [16]. As mentioned above, the quality of the interface also weighs in 
on the measured values as demonstrated by the increase in $h_{\mathrm{K}}$ for $\mathrm{Al} / \mathrm{Si}$ interfaces when the ( $\sim 2 \mathrm{~nm}$ thickness) native oxide layer at the interface is removed [17]. Taken together, the measured values of $h_{\mathrm{K}}$ shown in Fig. 1.1 suggests that several factors need to be considered to fully understand the driving mechanisms behind interfacial thermal transport.

Understanding $h_{\mathrm{K}}$ measured for metal/nonmetal interfaces has been a subject of debate over the past few decades. Stoner and Maris [19, 25] reported on the measurements of $h_{\mathrm{K}}$ between a series of metals and dielectrics; Figure 1.1 shows their measurements on $\mathrm{Pb}$ /diamond interface. They compared their experimental findings to various $\mathrm{p}-\mathrm{p}$ scattering models [26] and found discrepancies for interfaces with soft metals ( $\mathrm{Ag}$ and $\mathrm{Pb}$ ). Their findings led Huberman and Overhauser [27] and Sergeev [28, 29] to propose a different channel for energy conduction, i.e., metal electrons directly interacting with the non-metal phonons.

Since Stoner and Maris' seminal works [19, 25], Lyeo and Cahill have shown that electron-interface scattering does not significantly contribute to the thermal boundary conductance at metal/nonmetal interfaces by showing the $h_{\mathrm{K}}$ across $\mathrm{Pb}$ /diamond and $\mathrm{Bi} / \mathrm{diamond}$ interfaces are very similar due to the similar Debye temperatures for the two metals even though they have vastly different electron densities around their respective Fermi surfaces (c.f., Fig. 1.1). More recently, by considering metal/diamond interfaces at elevated pressures, Hohensee et al. [30] have also demonstrated that the primary mechanism driving $h_{\mathrm{K}}$ across metal/diamond interface is multiple phonon scattering processes near the interface. Similarly, previous work done from our group has also alluded to similar conclusions by considering various $p-p$ scattering theories $[31-33]$. This supports the e-p interfacial mechanism proposed by Majumdar and Reddy [34] in which e-p coupling in the bulk of the metal must occur before p-p scattering transmits energy across a metal/non-metal interface, and thus, heat conduction across the interface is not driven by e-p coupling mechanisms.

Contrary to the previous paragraphs discussions, there have been experimental works that demonstrate electon-interface scattering can change the overall rate of e-p equilibration of a thin Au film when the electrons have a different energy density than the lattice [35-37]. 
These works speculated that when the film thickness is less than the e-p mean free path, electron-interface scattering can result in an increase in e-p coupling [35-37]. An additional finding from these works determined that as the degree of e-p non-equilibrium increases (i.e., as the electron temperature increases and $T_{e}-T_{0} \gg T_{0}$ ), the electron-interface scattering mechanism increases. This suggests an additional channel for thermal transport across interfaces that are solely driven by the scattering of metal electrons on the interface and exchanging energy to the non-metal substrate.

Along with electron-boundary scattering, the nonequilibrium electron dynamics in laser heated metals presents a plethora of fundamental physics questions that need further consideration. For example, typically after short-pulsed excitation of a thin metal film on a dielectric substrate, the electron gas in a metal can be heated to several thousand degrees above the lattice temperature due to the large differences in the carriers' heat capacities. Understanding the implications of nonequilibrium e-e and e-p coupling mechanisms that occur during the 1-10 ps time regime at the metal/dielectric interface, and how these mechanisms affect the energy coupling across the interface that occurs on a $100 \mathrm{ps}$ - ns time scale is crucial in mitigating interfacial heat flow in high frequency solid state devices [6].

Where the above-mentioned physics of electronic relaxation typically dictates heat transfer in metallic systems, in semiconductor-based devices, $\mathrm{p}$-p mediated processes are the dominant energy relaxation mechanisms. Similar to electron-boundary scattering effects, vibrational scattering at the material boundary or a solid-solid interface has provided the opportunity for user-defined thermal transport in semiconductor-based devices. For example, in thermoelectric devices where low thermal conductivity materials are sought, high density of interfaces in SL structures can be utilized to severely limit thermal transport in the cross plane direction and therefore increase the overall figure of merit [38, 39].

While the TBC across inorganic-based interfaces has been widely studied (c.f., Fig.1.1), the knowledge of heat transfer across inorganic/organic interfaces has been mostly limited to $\mathrm{Au} / \mathrm{self}$-assembled monolayers (SAM) interfaces. In this context, there exists a void in literature on the study of thermal properties of hybrid materials and measurements of TBC 

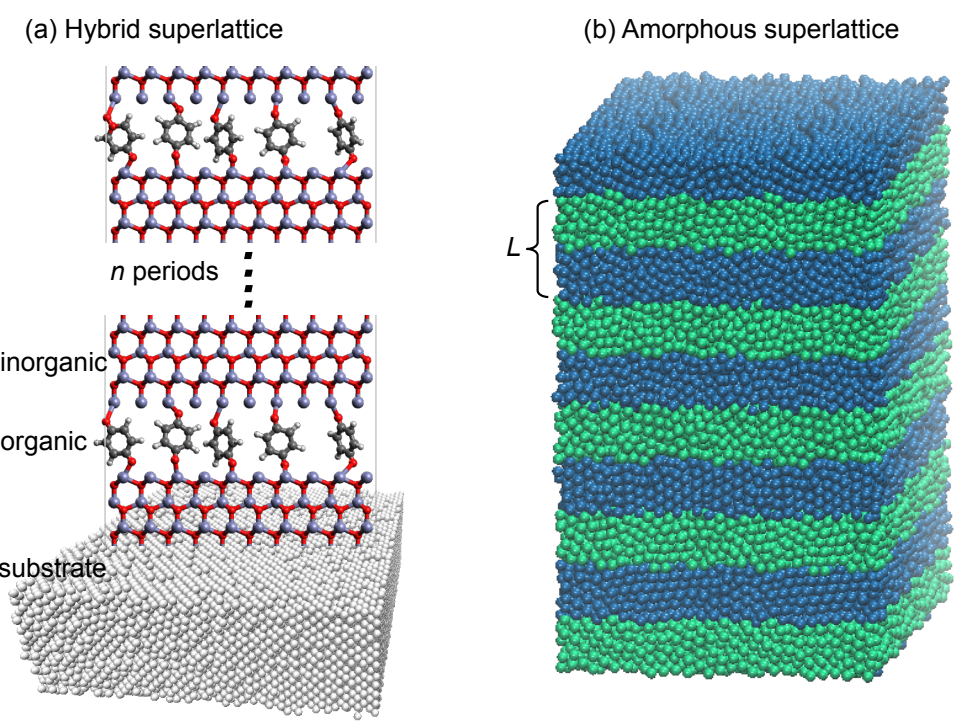

Figure 1.2: Illustrations of (a) hybrid superlattices with periodic inclusion of hydroquinone molecules inbetween $\mathrm{ZnO}$ inorganic constituents, and (b) amorphous superlattices with period length $L$ comprised of alternating layers of inorganic constituents.

across inorganic/organic interfaces. Similarly, thermal properties of inorganic-based semiconductor SLs have been widely studied in the past, whereas, the knowledge of thermal transport across hybrid inorganic/organic SLs still remains unexplored. Integrated with their enhanced electrical, optical, magnetic and mechanical properties in comparison to their conventional organic or inorganic counterparts, hybrid nanomaterials make for attractive candidates for a wide array of applications in nanotechnology [40-43]. An illustration of a hybrid superlattice comprised of organic layers of hydroquinone and inorganic layers of $\mathrm{ZnO}$ (which will be studied in Chapter 4) is depicted in Fig. 1.2 a. The organic layers are fabricated via the MLD process while the inorganic layers are fabricated via the ALD process. For these ALD/MLD grown hybrid superlattices, there have been limited previous works focusing on measurements of thermal properties [44]. Therefore, the advancement in the knowledge of the thermal properties in these hybrid nanostructures is necessary for a complete understanding of their materials physics.

Going back to Fig. 1.1. most of the measurements of TBC so far have been for interfaces with crystalline constituents; reports on amorphous-based interfaces has been non-existent. 
Similarly, amorphous-based SLs (as illustrated in Fig. $1.2 \mathrm{p}$ ) have received far less attention compared to their cyrstalline counterparts from the nanoscale heat transfer community. Even though the electrical and optical properties of amorphous semiconductor SLs have been extensively studied, their thermal conductivity is usually assumed to be a weighted average of the amorphous constituents since amorphicity in the periodic layers is thought to dictate thermal transport while interfaces are not considered to pose significant resistance to heat flow [45, 46]. This has limited the measurement of $h_{\mathrm{K}}$ across interfaces with amorphous constituents and therefore a study focusing on measuring the thermal properties of amorphous-based SLs, with an emphasis on understanding the interfacial thermal transport would mark a major contribution to the field of heat transfer mechanisms in SL structures as well as augment the fundamental understanding of vibrational coupling across interfaces in amorphous materials.

\subsection{Outline and objectives}

The overarching objective of this dissertation is to enhance the field's understanding of the fundamental dynamics of energy carriers across solid interfaces (i.e., the electronelectron (e-e), electron-phonon (e-p) and phonon-phonon (p-p) coupling mechanisms) in various nanosystems with multiple layers and interfaces (e.g., thin films at interfaces and SLs), which occur at different time scales. Both experimental as well as computational techniques will be implemented throughout this dissertation to understand the dynamics of energy carriers. The dissertation is separated into the following six chapters:

- Chapter 2, Interfacial transport: theory and experiment: Fundamental physics governing interfacial heat flow at various solid/solid interfaces is discussed, with particular attention given to the development of a theoretical framework studying the dynamics of electrons in a metal directly coupling to phonons in a nonmetal. Nonequillibrium molecular dynamics simulation is introduced and utilized to understand interfacial transport between two Lennard-Jones based solids. The time domain ther- 
moreflectance (TDTR) technique and the analysis procedures implemented to experimentally study interfacial transport for various material systems in this work are also introduced.

- Chapter 3, Mechanisms of electron-phonon coupling at interfaces: Nonequilibrium e-p processes at metal/dielectric interfaces are probed via TDTR. Utilizing the various time regimes measured during a TDTR experiment and implementing the appropriate analysis methods outlined in the previous chapter, a thorough understanding of the different factors that affect direct coupling between electrons in a metal and phonons in a nonmetal across an interface is developed.

- Chapter 4, Thermal transport in hybrid superlattices: A systematic study of thermal transport in hybrid superlattices is presented. Thermophysical quantities such as thermal conductivity and heat capacity of the hybrid superlattices are measured via TDTR. Along with that, the thermal boundary conductances associated with the thin film as well across inorganic/organic/inorganic boundaries is measured and the fundamental driving mechanisms behind these measurements are thoroughly analyzed. New insights into the underlying physics of thermal transport in hybrid superlattices are discussed and their implications towards controlling the tunability in energy storage and transport in these novel materials is emphasized.

- Chapter 5, Thermal transport across amorphous-based confined thin films and superlattices: Nonequilibrium molecular dyanims simulations are implemented to investigate the heat transport across disordered thin films sandwiched between crystalline materials. The effect of interface density on fully amorphous superlattices is studied experimentally as well as computationally. The results from these simulations and experiments lead to the design of amorphous/crystalline superlattices with ultralow thermal conductivities with a potential to advance thermoelectric applications.

- Chapter 6, Conclusions and future work: Major conclusions from the original works presented in the dissertation are discussed. Potential projects and outlook on future 
works deriving from these original works are proposed. 


\section{Chapter 2}

\section{Interfacial transport: theory and}

\section{experiment}

\subsection{Interfacial thermal transport}

A mathematical representation of heat flux across an interface from side 1 to side 2 , in the most general form, can be given in terms of the Landauer formalism as [47],

$$
q_{z}^{1 \rightarrow 2}=\frac{1}{(2 \pi)^{3}} \sum_{j} \int_{0}^{\pi / 2} \int_{0}^{2 \pi} \int_{k_{x, 1}} \int_{k_{y, 1}} \int_{k_{z, 1}>0} E_{1} v_{j, 1} \cos \theta_{1} \sin \theta_{1} f \zeta^{1 \rightarrow 2} d k_{x} d k_{y} d k_{z} d \theta_{1} d \phi_{1}
$$

where the transport is in the $z$-direction, $j$ is the polarization, $\theta_{1}$ and $\phi_{1}$ are the azimuthal and elevation angles of the heat flux, $k$ is the wave-vector, $\zeta^{1 \rightarrow 2}$ is the transmission coefficient from side 1 to $2, f$ is the distribution function for the energy carrier, $v_{j}$ is the carrier group velocity. Solving the full Eq. 2.1 to calculate interfacial flux requires knowledge of spectral contributions from the energy carriers. Futhermore, due to the fact that thermal transport occurs when the system is driven out of equilibrium, it is technically incorrect to assume that the energy distribution can be approximated with an equilibrium distribution function such as the Fermi-Dirac or Bose-Einstein distributions. In this context, the full Boltzmann transport equation needs to be solved to formulate $f$, which requires large com- 
putational resources. To reduce the computational cost, simplified statistical distribution functions are used and general assumptions regarding interfacial scattering are invoked to effectively predict the flux across interfaces between two materials. For example, the phonon flux impingent on an interface between two isotropic solids can be approximated under the isotropic assumption as,

$$
q=\frac{1}{8 \pi^{3}} \sum_{j} \int_{k} \hbar \omega(k) D(k) f_{0}(k, T) v_{g, j}(k) d k
$$

where $f_{0}$ is described by the equilibrium Bose-Einstein distribution function, $f_{0}=1 /\left(\exp \left(\hbar \omega / k_{B} T\right)-1\right)$. The temperature derivative of Eq. 2.2 with the inclusion of a transmission coefficient allows for the calculation of thermal boundary conductance, which is given as,

$$
h_{\mathrm{K}}=\frac{1}{8 \pi^{3}} \sum_{j} \int_{k} \hbar \omega(k) D(k) \frac{\partial f_{0}(k, T)}{\partial T} v_{g, j}(k) \zeta(k)^{1 \rightarrow 2} d k .
$$

The transmission coefficient from side 1 to $2, \zeta(k)^{1 \rightarrow 2}$, can be approximated via various p-p scattering models such as the diffuse mismatch model (DMM) and acoustic mismatch model (AMM) [22, 26, 48]). In these models, the mismatch in acoustic properties or vibrational density of states, limits the interfacial phonon transmission, and therefore restricts the phonon flux that transmits across the interface. As these models and further refinements of these models have been extensively described in the literature [32, 33, 47, 49, 53], these derivations will not be reproduced here. Instead, an analytical formulation of a coupled DMM and quantum mechanical derivation of electron-phonon scattering at free electron metal/nonmetal substrate interfaces will be derived in the following subsection. The analytical formulation will be used later in the following chapter to help explain some of the experimental findings on energy transfer across various metal film/dielectric substrate interfaces. Parts of section 2.2 are published in Journal of Heat Transfer, "Influence of Hot Electron Scattering and Electron-Phonon Interactions on Thermal Boundary Conductance at Metal/Nonmetal Interfaces" [54]. A large portion of section 2.4 is presented in Journal 
of Physical Chemistry C, "Implications of Interfacial Bond Strength on the Spectral Contributions to Thermal Boundary Conductance Across Solid, Liquid and Gas Interfaces: A Molecular Dynamics Study" [55].

\subsection{Diffuse mismatch model for electron energy exchange at a metal/nonmetal interface}

Due to their femtosecond resolution, short-pulsed lasers have been widely used to understand the scattering mechanisms of the fundamental energy carriers. To quantitatively predict these highly nonequilibrium processes in metals, theoretical and computational studies have employed the two-temperature model [56] that describes the temporal and spatial evolution of the electronic and lattice temperatures during ultrafast laser heating. The absorption of the laser pulse by the metal surface and the subsequent energy relaxation processes thereafter can be described by three characteristic time intervals: i) the thermalization of the free electron gas, ii) the coupling between electrons and the lattice; and iii) the energy transport driven by the gradient in the lattice temperature [57].

The metal electrons are defined by a range of energies resulting from the equivalent equilibrium temperature of the thermalized electrons and degree of Fermi smearing. In a free electron metal, the vast majority of excited electrons that are losing energy to the lattice are on the Fermi surface with energies of some $\delta \varepsilon$ around the Fermi energy. For free electron metals with a relatively constant density of states around the Fermi energy, $\delta \varepsilon=\hbar \omega \ll \Delta \varepsilon_{F}$, where $\varepsilon_{F}$ is the Fermi energy and $\Delta \varepsilon_{F}$ is the width of the constant energy density of states around the Fermi energy (for example, assuming gold electrons interacting with silicon substrate phonons, the maximum phonon energy available for metal-electron energy interaction is $\hbar \omega \approx h(15 \mathrm{THz}) \approx 0.06 \mathrm{eV}$ which is much less than the Fermi level to $5 d^{10}$-band edge energy separation in gold, $\Delta \varepsilon_{F} \approx 1.8 \mathrm{eV}$ ) [58]. Therefore, the electron energy and population interacting with the phonons in a free electron noble metal will not deviate from those at the Fermi energy as the energy states of electrons before and after the 
collisions are very close.

Assuming an isotropic metal, the electron-substrate energy transfer across the interface is given by,

$$
h_{\mathrm{es}}=\frac{1}{4} \int_{\varepsilon}\left(\varepsilon-\varepsilon_{F}\right) D_{e}(\varepsilon) \frac{\partial f}{\partial T_{\mathrm{e}, \mathrm{eff}}} v_{f} \zeta_{\text {int }} d \varepsilon
$$

where $\varepsilon$ is the electron energy, $D_{e}$ is the density of states (DOS) for electrons, $v_{f}$ is the Fermi velocity, $f$ is the electron distribution function, and $\zeta_{\text {int }}$ is the transmission coefficient. The critical parameter in calculating the thermal boundary conductance is the transmission coefficient.

Electron-phonon (e-p) scattering processes involve the redistribution of electrons among the allowed energy states by either emitting or absorbing phonons. During this process, electrons can be forced into or out of an energy state. Assuming single phonon emission or absorption from e-p collisions [59], the energy of the electrons and the subsequent phonons can be well defined. The transition probabilities from an electron distribution $f$ to a different distribution $f^{\prime}$ by emitting a phonon is proportional to $f\left(1-f^{\prime}\right)(N+1)$, where $N$ describes the Bose-Einstein distribution of phonons with a particular vibrational frequency. The $N+1$ term represents the phonon emission probability that includes both the stimulated and spontaneous emission. Similarly, the transition probability that involves absorption of a phonon by an electron distribution $f$ to a distribution $f^{\prime}$ is proportional to $f\left(1-f^{\prime}\right)(N)$. The total rate of change of electron density in a particular distribution $f$ can most rigorously be derived with Fermi's Golden Rule and the e-p interaction matrix over all energy states [60]. However, Qiu and Tien [59] offered a simple three-level electronic transition picture to calculate the e-p interaction probability based on the electron and phonon distributions at different energies. This approach agreed with Kaganov et al.'s [61] approach and is therefore adopted here.

Following Qiu and Tien [59], it is assumed that electronic transitions take place between three energy levels: $\varepsilon_{F}-\hbar \omega, \varepsilon_{F}$, and $\varepsilon_{F}+\hbar \omega$. Considering that $\delta \varepsilon \ll \varepsilon_{F}$, these are reasonable assumptions. With these transitions, one can then define the total electronic transition probability as the sum of each individual transition probability in our simple three-level 


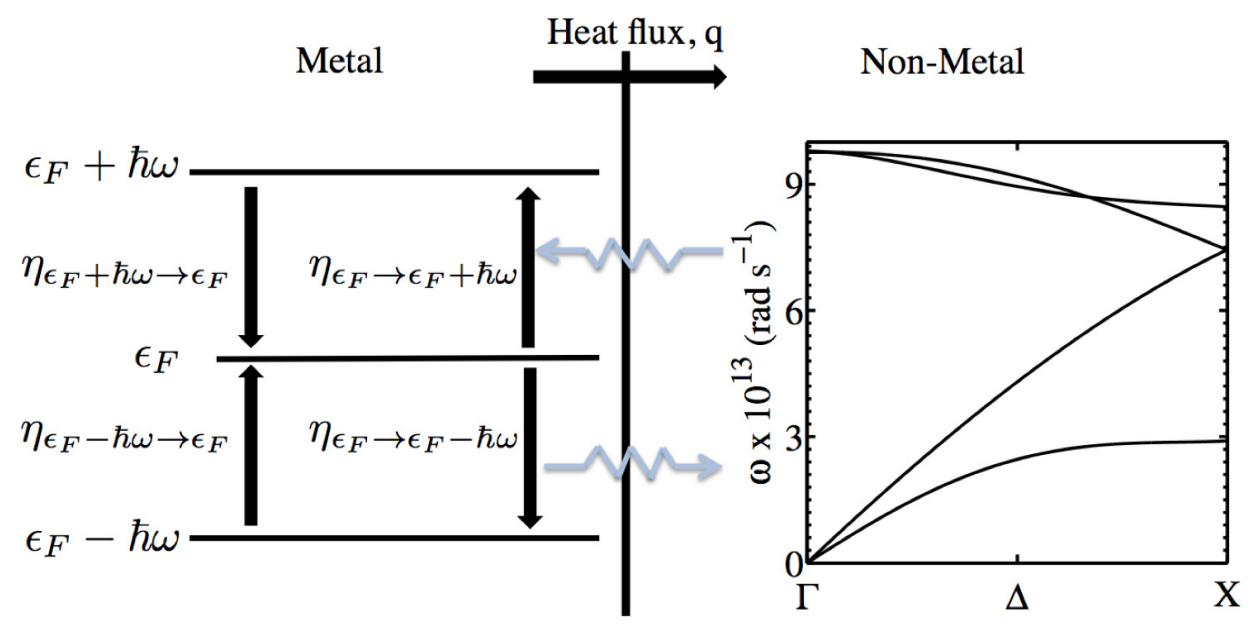

Figure 2.1: Schematic diagram showing the transitions in the three energy levels in the metal and phonon frequencies in the non-metal.

system [59], given by

$$
\zeta_{i n t}(\omega)=\eta_{\varepsilon_{F}-\hbar \omega \rightarrow \varepsilon_{F}}(\omega)+\eta_{\varepsilon_{F}+\hbar \omega \rightarrow \varepsilon_{F}}(\omega)+\eta_{\varepsilon_{F} \rightarrow \varepsilon_{F}-\hbar \omega}(\omega)+\eta_{\varepsilon_{F} \rightarrow \varepsilon_{F}+\hbar \omega}(\omega)
$$

where $\eta_{\varepsilon_{F}-\hbar \omega \rightarrow \varepsilon_{F}}$ is the probability of an electronic transition from the $\varepsilon_{F}-\hbar \omega$ energy level to the Fermi energy, $\varepsilon_{F}$. The other probabilities are defined similarly based on the subscripts of $\eta$. This is shown schematically in Fig. 2.1

Phonon absorption in the metal causes an electronic transition from a lower to a higher energy level where phonon emission from the metal causes an electronic transition from a higher to a lower energy level. These electronic transition probabilities ( $\eta_{A}$ for phonon absorption and $\eta_{E}$ for phonon emission) are related to the occupancy of the electronic energy levels. Therefore, the transition probability from energy $\varepsilon_{F}+\hbar \omega$ to $\varepsilon_{F}$ by emitting a phonon is

$$
\eta_{\varepsilon_{F}+\hbar \omega \rightarrow \varepsilon_{F}}(\omega)=f\left(\varepsilon_{F}+\hbar \omega\right)\left[1-f\left(\varepsilon_{F}\right)\right] \xi_{E}=\frac{\xi_{E}}{2\left(1+\exp \left(\frac{\hbar \omega}{k_{B} T_{\mathrm{e}, \mathrm{eff}}}\right)\right)}
$$


where $\xi_{E}$ is the probability of emitting a phonon [59]. Similarly,

$$
\eta_{\varepsilon_{F}-\hbar \omega \rightarrow \varepsilon_{F}}(\omega)=f\left(\varepsilon_{F}-\hbar \omega\right)\left[1-f\left(\varepsilon_{F}\right)\right] \xi_{A}=\frac{\xi_{A}}{2\left(1+\exp \left(-\frac{\hbar \omega}{k_{B} T_{\mathrm{e}, \mathrm{eff}}}\right)\right)}
$$

where $\xi_{A}$ is the probability of absorbing a phonon. Note that $\eta_{\mathcal{\varepsilon}-\hbar \omega \rightarrow \varepsilon_{F}}=\eta_{\varepsilon_{F} \rightarrow \varepsilon_{F}+\hbar \omega}$ and $\eta_{\varepsilon+\hbar \omega \rightarrow \varepsilon_{F}}=\eta_{\varepsilon_{F} \rightarrow \varepsilon_{F}-\hbar \omega}$ for the same phonon frequency, $\omega$.

In a homogeneous material, the probability of absorption or emission of a phonon from e-p collisions is given by [59]

$$
\xi_{A}=\frac{1}{\exp \left(\frac{\hbar \omega}{k_{B} T_{0}}+1\right)}
$$

and

$$
\xi_{E}=\frac{\exp \frac{\hbar \omega}{k_{B} T_{0}}}{\exp \left(\frac{\hbar \omega}{k_{B} T_{0}}+1\right)}
$$

respectively, where $\xi_{A} \equiv 1-\xi_{E}$ for a homogeneous material when $T_{\mathrm{e}, \mathrm{eff}}=T_{0}$. At an interface between a metal and non-metal, these probabilities are different. Therefore, $\xi_{A}$ and $\xi_{E}$ must be redefined for the interface problem.

As mentioned in the earlier section, the transmission probability of heat fluxes at solid interfaces has been addressed specifically for electron-electron (e-e) [14, 62] and phononphonon [22] interactions for non-cryogenic temperature regimes by considering diffusive scattering of energy carriers [22]. In this approach, the carriers are assumed to scatter diffusively and the transmission probability is determined by equating the thermal fluxes on either side of the interface via the Principle of Detailed Balance (in-depth treatments regarding these assumptions are discussed in Refs. [47, 63]). In short, the fundamental assumption of diffuse scattering implies that incident carriers lose all memory of their initial direction after scattering at the interface. For each $\omega$, one can equate the heat fluxes on 
either side given by,

$$
\begin{array}{r}
\hbar \omega\left(D_{p, L}\left(k_{L}\right)+2 D_{p, T}\left(k_{T}\right)\right) v_{f} f\left(\varepsilon_{F}-\hbar \omega, T_{\mathrm{e}, \mathrm{eff}}\right) \xi_{m, E} \\
=\hbar \omega\left(N\left(\hbar \omega, T_{0}\right)+1\right)\left(D_{p, L}\left(k_{L}\right) v_{g, L}+2 D_{p, T}\left(k_{T}\right) v_{g, T}\right) \xi_{n m, A},
\end{array}
$$

which relates the phonon emission via metal-electron relaxation to phonon absorption in the non-metal and

$$
\begin{aligned}
& \hbar \omega\left(D_{p, L}\left(k_{L}\right)+2 D_{p, T}\left(k_{T}\right)\right) v_{f} f\left(\varepsilon_{F}+\hbar \omega, T_{\mathrm{e}, \mathrm{eff}}\right) \xi_{m, A} \\
= & \hbar \omega N\left(\hbar \omega, T_{0}\right)\left(D_{p, L}\left(k_{L}\right) v_{g, L}+2 D_{p, T}\left(k_{T}\right) v_{g, T}\right) \xi_{n m, E},
\end{aligned}
$$

which relates the phonon absorption via metal-electron excitation to phonon emission in the non- metal. In these expressions, $D_{p, L}$ and $D_{p, T}$ are the densities of states for longitudinal and transverse mode phonons in the non-metal, respectively, $k_{L}$ and $k_{T}$ are the wave-vectors for the corresponding longitudinal and transverse phonon frequencies, respectively, $v_{g, L}$ and $v_{g, T}$ are the longitudinal and transverse phonon group velocities, respectively, and $N$ is the Bose-Einstein distribution function. The $\xi_{(m, E),(n m, A),(m, A),(n m, E)}$ terms are the probability of emission $(E)$ and absorption $(A)$ by metal $(m)$ and non-metal $(n m)$. The $D_{p, j}$ terms appear on the flux from the metal side due to our assumption of single phonon absorption or emission during the collision process; in other words, the number of quantum states and energy of electrons in the metal interacting with the non-metal must be equal to the number of quantum states and energy of phonons in the non-metal. The phonon density of states in the non-metal is given by

$$
D_{p, j}\left(k_{j}\right)=\frac{k_{j}^{2}}{2 \pi^{2} v_{g, j}}
$$

where the index $j$ represents the summation over all the polarizations of the phonons in the non-metal. From the assumption of diffuse scattering, we have $1-\xi_{m, E}=\xi_{n m, A}$ and $1-\xi_{m, A}=\xi_{n m, E}$. Therefore, Eqs. 2.10 and 2.11 can be rearranged to determine the metal 


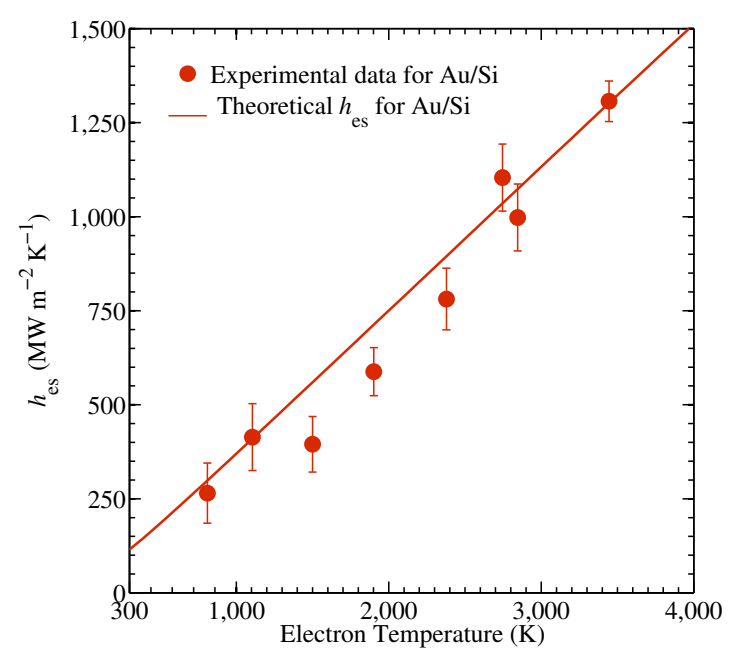

Figure 2.2: $h_{\mathrm{es}}$ predicted from the theoretical framework developed in this work compared to the experimentally determined $h_{\mathrm{es}}$ from the TTR data analyzed with the two temperature model [36]. The $h_{\mathrm{es}}$ increases linearly with increasing effective electron temperature.

emission and absorption probabilities at phonon frequency, $\omega$

$$
\xi_{m, E}\left(\omega, T_{\mathrm{e}, \mathrm{eff}}, T_{0}\right)=\frac{\left(N\left(\hbar \omega, T_{0}\right)+1\right)\left(k_{L}+2 k_{T}\right)}{v_{f} f\left(\varepsilon-\hbar \omega, T_{\mathrm{e}, \mathrm{eff}}\right)\left(\frac{k_{L}}{v_{g, L}}+\frac{2 k_{T}}{v_{g, T}}\right)+\left(N\left(\hbar \omega, T_{0}\right)+1\right)\left(k_{L}+2 k_{T}\right)}
$$

and

$$
\xi_{m, A}\left(\omega, T_{\mathrm{e}, \mathrm{eff}}, T_{0}\right)=\frac{N\left(\hbar \omega, T_{0}\right)\left(k_{L}+2 k_{T}\right)}{v_{f} f\left(\varepsilon+\hbar \omega, T_{\mathrm{e}, \mathrm{eff}}\right)\left(\frac{k_{L}}{v_{g, L}}+\frac{2 k_{T}}{v_{g, T}}\right)+N\left(\hbar \omega, T_{0}\right)\left(k_{L}+2 k_{T}\right)} .
$$

In these expressions, we assume two degenerate transverse phonon branches. Using Eqs. 2.13 and 2.14 with Eqs. 2.6 and 2.7, the spectral transition probability of electrons due to nonmetal phonon interaction can be determined via Eq. 2.5.

Figure 2.2 shows the calculations performed for $\mathrm{Au} / \mathrm{Si}$ interface compared to experimentally determined $h_{\text {es }}$ from the TTR data analyzed with the two temperature model in Ref. [36]. The calculations of Eq. 2.4 agree well with the experimental data, suggesting that at elevated electron temperatures, $h_{\mathrm{es}}$ can significantly affect thermal transport across 
$\mathrm{Au} / \mathrm{Si}$ interfaces [54]. Based on the agreement of the theoretical model and experimental data, the linear temperature dependence in $h_{\mathrm{es}}$ arises due to the energy of the electrons in the metal (i.e., the temperature dependence of the electronic heat capacity); this is similar to the conclusion found for the electron thermal boundary conductance across metal/metal interfaces [64]. The derivations presented above will be used in the following chapter to gauge whether the direct coupling of metal-electron/non-metal phonon can be considered a viable channel for energy transport across metal/nonmetal interfaces.

\subsection{Molecular dynamics simulations}

The classical molecular dynamics (MD) technique involves calculating the time evolution of the positions and energies of atoms in a computational domain through Newtonian mechanics. These atoms are treated as point masses that have no internal structure or degrees of freedom and their quantum nature is neglected. The output of the MD simulations are the kinetic energies of these atoms and relating these energies to the temperature, $T$, through the relationship,

$$
\frac{3}{2} N k_{\mathrm{B}} T=\sum_{i=1}^{N} \frac{1}{2} m_{i}\left(v_{i}^{T}\right)^{2}
$$

where $N$ is the number of particles, $k_{\mathrm{B}}$ is the Boltzmann constant, $m$ is the mass of the atoms and $v_{i}^{T}$ is the thermal velocity of the atoms, allows for the determination of the various thermal properties of interest.

The NEMD approach utilizes a thermal flux across the computational domain, which induces a steady-state temperature gradient. The temperature gradient is used to predict the thermal conductivity or the thermal boundary conductance between two different materials. If the thermal conductivity is sought, the observed spatial temperature gradient can be related to the thermal conductivity by invoking the Fourier law, $Q=-\kappa \partial T / \partial z$, where applied flux is in the z-direction. As for the thermal boundary resistance, a temperature discontinuity at an interface, $\Delta T$, is related to conductance through the relationship $Q=$ 
$R_{\mathrm{K}}^{-1} \Delta T$

The NEMD approach is particularly well suited for predictions of $R_{\mathrm{K}}\left(\right.$ or $h_{\mathrm{K}}^{-1}$ ) as the calculation of the temperature drop at the interface between two materials is very straight forward. In the following section, the NEMD routine (performed using the LAMMPS molecular dynamics package [65]) implemented in the thesis is used to predict $R_{\mathrm{K}}$ across an interface between two solids defined by the Lennard-Jones (LJ) potential. Even though material specific interatomic potentials will be utilized in the following chapters, the LJ potential used in this section serves to elucidate the general effects of strength of interfacial bonding on $R_{\mathrm{K}}$ at an acoustically mismatched interface. A large portion of the following section is taken from "Implications of interfacial bond strength on the spectral contributions to thermal boundary conductance across various phases of matter: A molecular dynamics study" [55].

\subsection{NEMD simulations on LJ-based solid/solid interfaces}

\subsubsection{Computational domain setup}

The solids are modeled by the 6-12 LJ potential, $U(r)=4 \varepsilon\left[(\sigma / r)^{12}-(\sigma / r)^{6}\right]$, where $U$ is the interatomic potential, $r$ is the interatomic separation, and $\sigma$ and $\varepsilon$ are the LJ length and energy parameters, respectively. For computational efficiency the cutoff distance is set to $2.5 \sigma$ for all the simulations and the time step is set to $0.1 \mathrm{fs}$ throughout the simulations. To gauge the effect of cross-species interaction strength on the spectral contribution to $h_{\mathrm{K}}$, structures are simulated with strong and weak interfacial bonding (defined by $\varepsilon$ for species across the interface).

As shown by the schematic in the top panel of Fig. 2.3, two materials (A and B) that are in contact with each other are considered for the NEMD simulations. The length and energy parameters are set to $\sigma_{\mathrm{Ar}}=3.405 \AA$ and $\varepsilon_{\mathrm{Ar}}=0.0103 \mathrm{eV}$, respectively (that is representative of solid Ar for both A and B). The materials are arranged in an fcc lattice with the same zero-temperature lattice constant of $a_{0}=1.55 \sigma$. This creates a lattice matched 


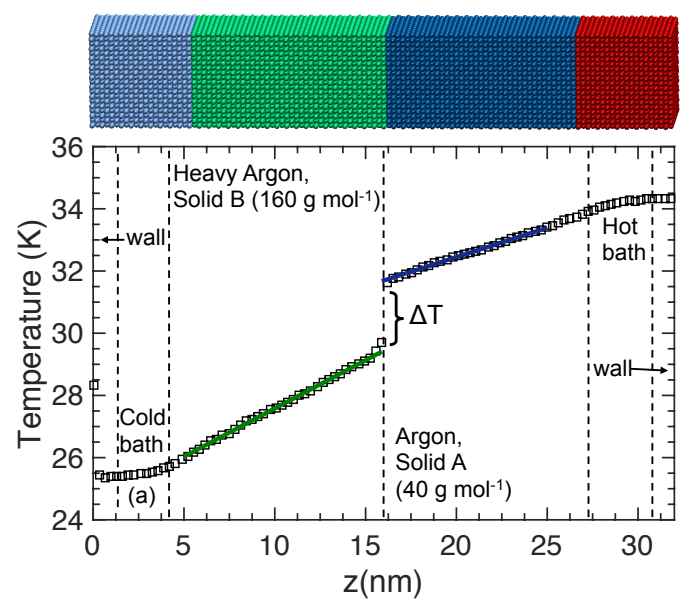

Figure 2.3: (Top panel) Atomistic illustration of the computational domain between LJbased solid/solid system. (Bottom panel)Temperature profile after a steady heat flux is imposed.

interface between materials A and B. However, an acoustic mismatch is introduced by setting the mass of material B to be 4 times higher than that of material A $\left(m_{\mathrm{B}}=4 m_{\mathrm{A}}\right)$. Thus, material A (with $m_{\mathrm{A}}=40 \mathrm{~g} \mathrm{~mol}^{-1}$ ) represents solid argon and material B represents a fictitiously heavier solid argon. The systems consist of $10 a_{0} \times 10 a_{0} \times 60 a_{0}$ computational domains with 24,000 atoms each. Note, increasing the cross-sectional area or the length of the computational domain has negligible change in the observed results. To investigate the effect of a weakly bonded interface, we change the energy parameter $\left(\varepsilon_{A r}\right)$ for interaction between the $\mathrm{A}$ and $\mathrm{B}$ materials to $\varepsilon_{\mathrm{AB}}=\varepsilon_{\mathrm{Ar}} / 4$. Periodic boundary conditions are used in the $x$ - and $y$-directions, whereas, a fixed boundary with 4 monolayers of atoms at each end are placed in the $z$-direction. Initially the structures are allowed to equilibrate at their prescribed temperatures for a total of $2 \mathrm{~ns}$ under the Nose-Hoover thermostat [66] (that is the NVT integration with the number of atoms, volume and temperature of the simulation held constant) followed by the NPT integration (which is the isothermal-isobaric ensemble with the number of particles, pressure and temperature of the system held constant) for a total of $2 \mathrm{~ns}$ at 0 bar pressure. After equilibration, a steady-state temperature gradient is established under the NVE integration (with number of particles, volume and energy held constant) by adding a fixed amount of energy per time step to a warm bath at one end and removing the 
equal amount of energy from a cool bath at the other end as shown in Fig. 2.3. The length of the baths are $10 a_{0}$ in the $z$-direction and are shown in the schematic in Fig. 2.3 as red (hot bath) and light blue (cold bath) atoms. A steady-state temperature profile is shown in the bottom panel of Fig. 2.3 .

\subsubsection{Density of states calculations and modal analysis}

After equilibration, the velocities of a group of atoms in either solid were recorded every 10 time steps for a total of $1 \times 10^{5}$ time steps using a NVE scheme to create a velocity fluctuation time series [67]. The density of states, $D(\omega)$, is obtained from the Fourier Transform $(\mathscr{F})$ of the velocity autocorrelation function $(V A C F)[68]$. The Welch method of power spectral density estimation is applied to obtain the $D(\omega)$ and is normalized as follows,

$$
D(\omega)=\frac{1}{2} m \mathscr{F}(V A C F) \frac{1}{k_{\mathrm{B}} T} \rho,
$$

where $\mathrm{m}$ is the atomic mass, $k_{\mathrm{B}}$ is the Boltzmann constant, $T$ is the local temperature, and $\rho$ is the atomic density. The density of states calculations using the outlined method for the LJ-based solids A and B are shown in Fig. 2.5. The heavier solid $\left(160 \mathrm{~g} \mathrm{~mol}^{-1}\right)$ has a lower cutoff frequency as compared to that of LJ-Ar due to the relation, $\omega \propto 1 / \sqrt{m}$.

The NEMD procedure or the DOS calculations outlined above do not directly lend insight into the modal contributions to $h_{\mathrm{K}}$ at an interface. Therefore, to quantify the spectral contributions to $h_{\mathrm{K}}$, we apply a similar method to that detailed in Ref. [69], where the correlations between the force-velocity at the interfaces is used to predict mode level details to understanding interfacial heat current. Briefly, the heat flux is spectrally resolved by the relation [69],

$$
Q=\int_{0}^{\infty} \frac{d \omega}{2 \pi} q(\omega)
$$


where $\omega$ is the angular frequency and $q(\omega)$ is the spectral heat current. In general, this heat current between an atom $i$ and $j$ is proportional to the correlation between the interatomic force $\vec{F}_{i j}$ between the atoms and the velocities, $q_{i \rightarrow j}(\omega) \propto\left\langle\vec{F}_{i j} \cdot\left(\vec{v}_{i}+\vec{v}_{j}\right)\right\rangle$, where the brakets denote steady-state nonequilibrium ensemble average [70-72]. Since the spectral contribution of the flux is to be calculated across a planar interface between solid/solid systems, calculations of the force exerted on atoms in each monolayer of solid (within the cutoff distance from the interface) on the left side of the interface due to the different species of atoms at the other side of the interface is performed. For the purposes of this work, atomic velocities and forces are collected for a total of $10 \mathrm{~ns}$ during steady-state nonequilibrium conditions at a sampling interval of $10 \mathrm{fs}$ in order to determine the spectral contribution to the total heat flux.

Note, this method of calculating the total force on an atom on the left side of the interface due to the collective forces from all the atoms in the other side of the interface reduces the computational cost by a large extent. Furthermore, instead of considering forces on every atom $i$ at the left side of the interface, we consider an average force exerted on a plane of atoms (due to periodic boundary conditions on $x$ - and $y$-directions and) since atoms in a monolayer parallel to the interface will experience the same force due to the collective atoms from the other side of the interface. In this context, the approach outlined here can considerably reduces the computational time and cost for the modal analysis calculations compared to previous methods where the computational cost of storing the velocities and forces between each atom pair interaction during the simulation and taking the Fourier transforms require a large amount of storage space [73, 74]

To validate the modal decomposition method described above, NEMD calculations on $\mathrm{LJ}$ argon at $50 \mathrm{~K}$ are performed by placing an imaginary interface with a cross section at the middle of the simulation cell in the $z$-direction. For the calculations, the simulation domain size is $10 \mathrm{a}_{0} \times 10 \mathrm{a}_{0} \times 60 \mathrm{a}_{0}$, which confirms that no size effects due to boundary scattering affects the calculations. Figure 2.4 shows the modal contributions of the normalized heat flux accumulation $(q(\omega))$ due to a steady-state temperature induced across the LJ argon 


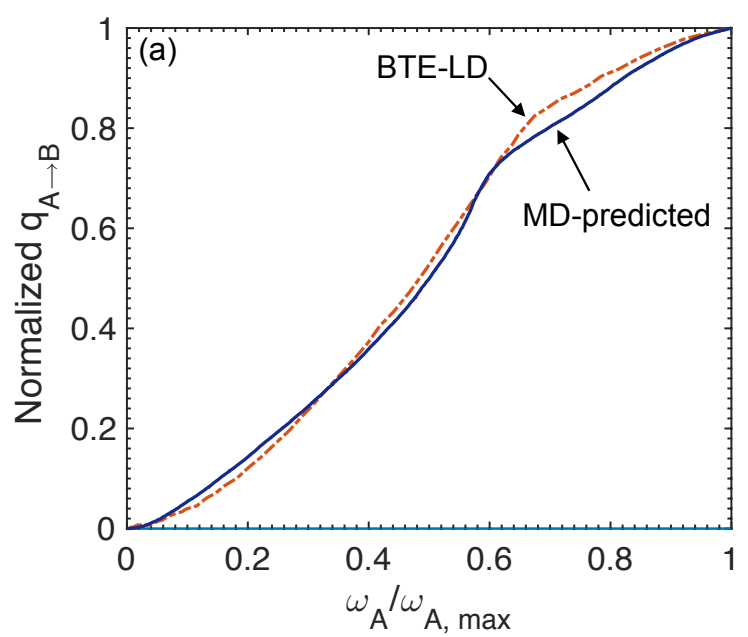

Figure 2.4: Normalized thermal conductivity accumulation predicted from the NEMD calculations for a homogeneous LJ argon with an imaginary interface in the middle of the computational domain (at $50 \mathrm{~K}$ ) as a function of $\omega_{\mathrm{Ar}} / \omega_{\mathrm{Ar} \text { max }}$. For comparison, the result from Ref. [75] that is based on the Boltzmann transport equation in conjunction with anharmonic lattice dynamics calculation performed at $50 \mathrm{~K}$ are also shown.

computational domain. For comparison, the predictions from a Boltzmann transport equation (BTE) in conjunction with anharmonic lattice dynamics (LD) calculations performed at $50 \mathrm{~K}$ (with the same LJ parameters as detailed in Ref. [75]) are also shown. In this method, the phonon properties predicted via anharmonic LD calculations, which takes into consideration three- and four-phonon processes, are used as input parameters in the BTE equation. The modal decomposition method and the BTE-LD method predict very similar spectral contributions to the thermal conductivity of LJ argon. In particular, both methods predict that the largest contribution to thermal conductivity is because of phonons with $20 \%$ to $80 \%$ of the maximum frequency. This is intuitive because of the large population of phonons in this frequency range (see the density-of-states of LJ argon represented by Solid A in Fig. 2.5 $)$. The good agreement between the two approaches provides confidence on the results presented in this section. Note that the BTE-LD approach and the approach used in this work are fundamentally different as the former uses information such as the relaxation times as input parameters in the BTE, while the approach outlined above relies 
on the outputs of atomic velocities and forces directly from MD simulations.

\subsubsection{Spectral contributions to thermal boundary conductance}
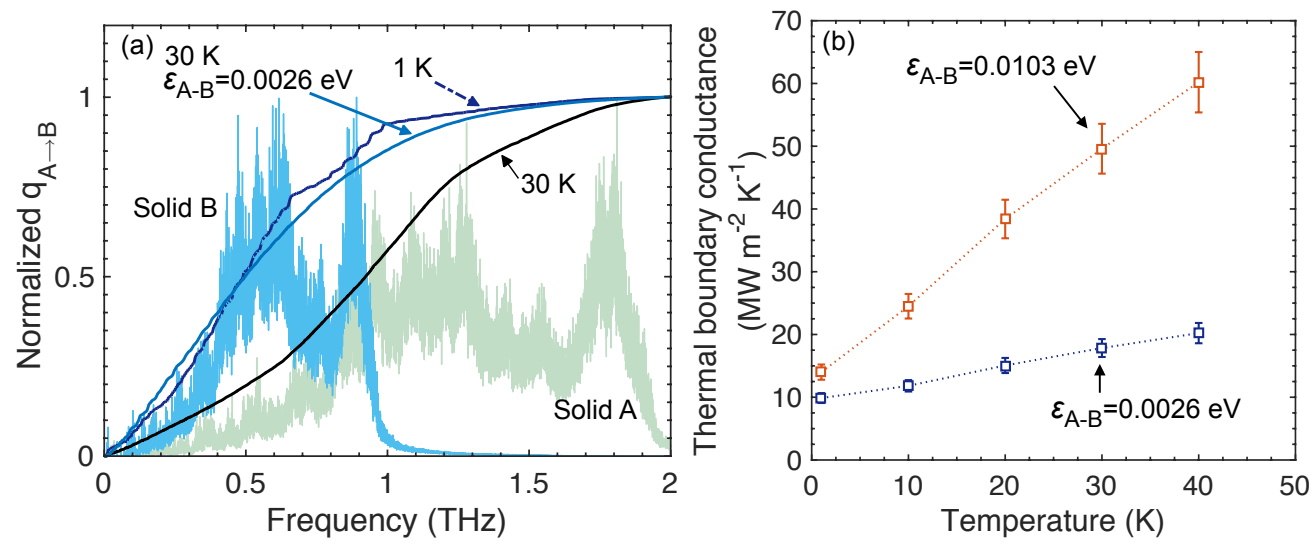

Figure 2.5: (a) Normalized thermal boundary accumulation at the interface of a two LJbased solids at $1 \mathrm{~K}$ and $30 \mathrm{~K}$ with $\varepsilon_{\mathrm{ss}}=0.0103 \mathrm{eV}$. Also included is the prediction for a weak cross-species interaction strength across the interface with $\varepsilon_{\mathrm{ss}}=0.0026 \mathrm{eV}$ at $30 \mathrm{~K}$. Also included are the DOS (a.u.) of the bulk solids. (b) NEMD-predicted thermal boundary conductances across the two LJ-based solids differentiated by mass as a function of temperature for strong and weak cross-species interaction.

Figure 2.5 a shows the modal contributions to the normalized heat flux accumulation from Solid A $\left(m=m_{\mathrm{Ar}}\right)$ to Solid B $\left(m=4 m_{\mathrm{Ar}}\right)$. At $1 \mathrm{~K}$ average temperature, the heat flux from Solid A to Solid B has negligible contributions from frequencies greater than the cutoff frequency of Solid B (even though the phonon spectrum in Solid A extends to twice the maximum frequency of Solid B). However, at $30 \mathrm{~K}$, frequencies greater than the cutoff frequency of Solid B contribute to more than $50 \%$ of the total heat flux from Solid A to Solid B. The temperature dependencies can be understood by considering inelastic channels and anharmonic phonon processes that are activated because of the increase in temperature. Where harmonic interactions limit the accumulation to reach $100 \%$ by $\omega_{\mathrm{B}}$, max , anharmonic interactions open up channels for heat conduction, thereby allowing modes with different frequencies to interact [18, 32, 33, 52, 69, 76-79]. Even though in homogeneous crystals, anharmonicity decreases thermal conductivity because of various scattering mechanims, 
heat conduction across interfaces is aided by anharmonicity as is suggested by the increase in the spectrum of frequencies in Solid A that can carry heat across the interface at higher temperatures.

Figure 2.5 a also shows the modal contributions of a weakly bonded interface between Solid A and Solid B (with $\varepsilon_{\mathrm{A}-\mathrm{B}}=0.0026 \mathrm{eV}$ ) at $30 \mathrm{~K}$. In comparison to the strongly bonded case (with $\varepsilon_{\mathrm{A}-\mathrm{B}}=0.0103 \mathrm{eV}$ ), the weak strength of the cross-species interaction leads to a shift from mid-frequency phonons dominating heat flow to lower frequencies contributing the most to the heat flux from Solid A to Solid B. Also, in contrast to the strongly bonded case at $30 \mathrm{~K}$, inelastic channels are inhibited because of the weak cross-species interaction strength as is evident from the negligible contribution of phonon frequencies higher than the cutoff frequency of Solid A to the total heat flux. This is further quantified by the temperature dependencies of $h_{\mathrm{K}}$ across the Solid A/Solid B interface for the weakly and strongly bonded interfaces (see Fig. 2.5b). The increase in $h_{\mathrm{K}}$ with temperature is less pronounced for the case of the weakly bonded interface, and the $h_{\mathrm{K}}$ values converge at low temperatures for the weakly bonded and strongly bonded interfaces. This suggests that anharmonic phonon scattering processes that increase $h_{\mathrm{K}}$ with temperature for the strongly bonded case are inhibited for the weakly bonded case and this inhibition is less pronounced at lower temperatures where inelastic interactions are limited, in line with the calculations of modal contributions shown in Fig. 2.5a.

The NEMD framework along with the DOS calculations will be implemented for various material systems investigated in the later chapters. The implications of interfacial bonding studied for the LJ-based models in this section will also be readdressed in the next chapter.

\subsection{Time domain thermoreflectance (TDTR)}

The measurement of thermal properties of various material systems presented in this thesis have been carried out with the TDTR technique. In general, thermomodulation tech- 
niques have been utilized for more than half a century to describe a wide variety of phenomena in materials physics. For example, the first observation of nonequilibrium between the electronic and the vibrational states in metals with short-pulsed TDTR was carried out by Eesley [80] who confirmed prior theories positing that electrons and the lattice can be described by two separate temperatures. It should be noted that this section does not describe the intricate details of the TDTR system, nor does it have the complete derivations of the analytical models used to interpret TDTR data as these details can be found elsewhere $[81-85]$. Instead, the pertinent details regarding the specific experiments performed and the advancements achieved in the analysis procedure to accurately and simultaneously measure more than two parameters (that is typical of a TDTR analysis) will be emphasized.

\subsubsection{Experimental setup}

For the measurements presented in this thesis, the basic idea of a pump-probe thermoreflectance technique hinges on creating a modulated heating event on a metal surface with an energetic pump beam. The temperature decay due to this heating event is then monitored using a time-delayed probe beam, which emanates from the same laser.

In our TDTR experimental setup, laser pulses emanate from a Ti:Sapphire oscillator with an $80 \mathrm{MHz}$ repetition rate and a central wavelength of $800 \mathrm{~nm}$. A shematic of the optical layout in our TDTR setup is shown in Fig. 2.6. A polarizing beam splitter (PBS) energetically splits the beam into pump and probe paths and the probe path is frequency doubled from 1.55 to $3.1 \mathrm{eV}$ utilizing a bismuth triborate crystal (BiBO). We measured the FWHM of the probe pulse to be $220 \pm 20$ fs via the frequency resolved optical gating technique [86] and the cross correlation between the pump and probe pulses was measured as $780 \pm 20 \mathrm{fs}$. The $1 / e^{2}$ radii of the pump and probe spot sizes after being focused through an objective were measured with a scanning slit optical beam profiler. Depending on the requirements of the experiments, the spot sizes can be independently modified by focusing through optical lens or using objectives with higher magnifications.

The train of ultra-short pump pulses (blue laser path shown in Fig. 2.6 a) thermally 

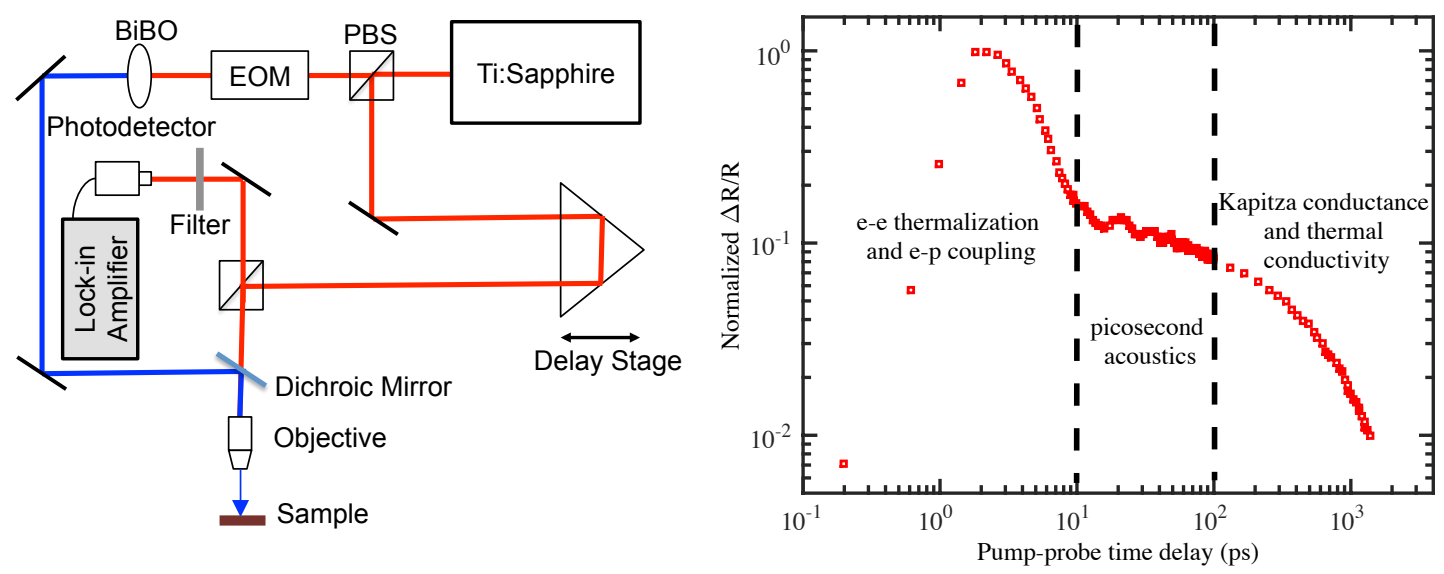

Figure 2.6: (a) Schematic of the two-color TDTR setup. (b) Thermoreflectance signal for a $20 \mathrm{~nm} \mathrm{Au} / \mathrm{Si}$ sample plotted as a function of delay time between the pump and probe pulses. The data shows three distinct time regimes; the initial $10 \mathrm{ps}$ during and after laser pulse absorption that is characterized by electronic thermalization in the Au film, followed by the picosecond acoustics regime that is marked by the periodic oscillatory signal caused by longitudinal displacement of, or a strain wave propagation in the film and the final time scale where the signal decays due to heat transport across the film substrate interface and and thermal effusion into the substrate. In this plot, 2 ps is arbitrarily chosen as the maximum signal.

stimulate the metal film and time delayed probe pulses (red laser path shown in Fig. 2.6a) measure the change in the thermoreflectance of the sample due to the decay of the deposited thermal energy. We modulate the pump path at various frequencies and monitor the in-phase $\left(V_{\text {in }}\right)$ and out-of-phase $\left(V_{\text {out }}\right)$ signals of the probe beam from a lock-in amplifier synced to those frequencies for up to $5 \mathrm{~ns}$ after the initial heating event.

A typical normalized magnitude of the in- and out-of-phase signals $\left(\sqrt{V_{\text {in }}^{2}+V_{\text {out }}^{2}}\right)$ for a thin $\mathrm{Au}$ film on a Si substrate is shown in Fig. 2.6p. Along with the magnitude, both $V_{\text {in }}$ and $V_{\text {out }}$ signals can be fit to the appropriate thermoreflectance model to measure the thermophysical properties, as will be made clear in the proceeding discussions. In short, the thermoreflectance signal relates the change in the base line reflectivity of a sample surface to the change in the temperature predicted by the various thermal models. More specifically, the change in reflectance of the metal transducer used in a typical measurement of thermal conductivity of a material can be related to the change in temperature through 
the change in the complex dielectric function.

For ultra-short pulses irradiated on a metal surface with $t_{\mathrm{p}}$ less than the e-p thermalization time in the metal, the dielectric function will depend on changes in both the electron and phonon temperatures. For small perturbations in temperature $(\Delta T \sim 150 \mathrm{~K})$, corresponding to low laser fluences, the change in reflectivity measured can be directly related to changes in electron and phonon temperatures through,

$$
\frac{\Delta R}{R}=a \Delta T_{\mathrm{e}}+b \Delta T_{\mathrm{p}}
$$

where $a \propto \partial R / \partial T_{\mathrm{e}}$ and $b \propto \partial R / \partial T_{\mathrm{p}}[87,88]$. At temperature excursions above $\sim 150 \mathrm{~K}$, the applicability of this thermoreflectance model breaks down and non-linear models such as the Drude-based reflectance model need to be considered [88].

As mentioned above, by fitting this "cooling curve" (represented by the decay in the thermoreflectance signal as shown in Fig. 2.6p) with various models in conjunction with a thermoreflectance model, the thermophysical properties of interest can be measured. For example, the e-p coupling factor, $G$, for a thin Au film deposited on a dielectric substrate can be determined by fitting the initial rise and the fast transient decay of the TDTR signal in the first few picoseconds (see Fig. 2.6p) to the two-temperature model (TTM) as discussed in detail below. The e-p nonequilibrium dynamics also governs the generation and propagation of acoustic-phonon pulses [89, 90] through the thin film, which is characterized by the oscillatory TDTR signal in the 10-100 ps time regime in Fig. 2.6b. The longer pump-probe delay times of up to several nanoseconds are generally fit with heat conduction models to back out the various thermal properties such as the thermal conductivity of the film and the substrate and the thermal boundary conductance [83-85]. Throughout this thesis, the different time scales represented in Fig. $2.6 \mathrm{p}$ will be analyzed with the appropriate thermal models to measure the desired physical properties of the material systems under study. 


\subsubsection{Thermal model and data analysis}

Typically in a TDTR experiment, the thermophysical properties of interest are the thermal conductivity $(\kappa)$ of a material and the thermal boundary conductance $\left(h_{\mathrm{K}}\right)$ across interfaces (such as the interface created by depositing an Al transducer layer on top of the material under study for TDTR experiments). To measure these properties, we analyze the data with a heat conduction model described through the conventional parabolic one-step heat diffusion equation

$$
C(T) \frac{\partial T}{\partial t}=\nabla \cdot(\kappa \nabla T)
$$

where $C(T)$ is the temperature dependent heat capacity. Due to the Gaussian geometries of the pump and probe beams and the fact that solving Eq. 2.19 in the time domain for multilayered structures is computationally very intensive, it is convenient to solve Eq. 2.19 in cylindrical coordinates and perform the analysis in the frequency domain, respectively [83]. It should be noted that the derivation of these equations are not reproduced here, however, the interested reader is referred to Ref. [82] for a comprehensive formulation of the equations.

Since, most of the work presented in this thesis deals with a three-layer model (layer 1: Al; layer 2: thin film; layer 3: semi-infinite substrate), the unknown parameters in the model are the TBCs across the front and back side of the thin film and the heat capacities and thermal conductivities of the film and the substrate. However, in a conventional TDTR analysis, only two parameters can be accurately measured. Therefore, it is very important to know which parameters in the thermal model can be independently determined in a TDTR measurement. For example, to evaluate the thermophysical properties of a $154 \mathrm{~nm} \mathrm{ZnO} \mathrm{film}$ grown on $\mathrm{Al}_{2} \mathrm{O}_{3}$ substrate via $\mathrm{ALD}$, namely the thermal boundary conductances across the $\mathrm{Al} / \mathrm{ZnO}$ and $\mathrm{ZnO} / \mathrm{Al}_{2} \mathrm{O}_{3}$ interfaces, the appropriate range of pump-probe delay times must be determined to fit the thermal model to the experimental data, in which the thermal model is extremely sensitive to changes in $h_{\mathrm{K}}[16,85]$; note, the $154 \mathrm{~nm} \mathrm{ZnO}$ film grown on $\mathrm{Al}_{2} \mathrm{O}_{3}$ will serve as a control sample for the study conducted for $\mathrm{ZnO}$-based hybrid superlattices 
in Chapter 4. With the proper sensitivity analysis described below, it will be shown that for the ALD grown $\mathrm{ZnO}$ film, the thermal conductivity cannot be measured with precision since the sensitivity to the measurement is dominated by the TBCs.

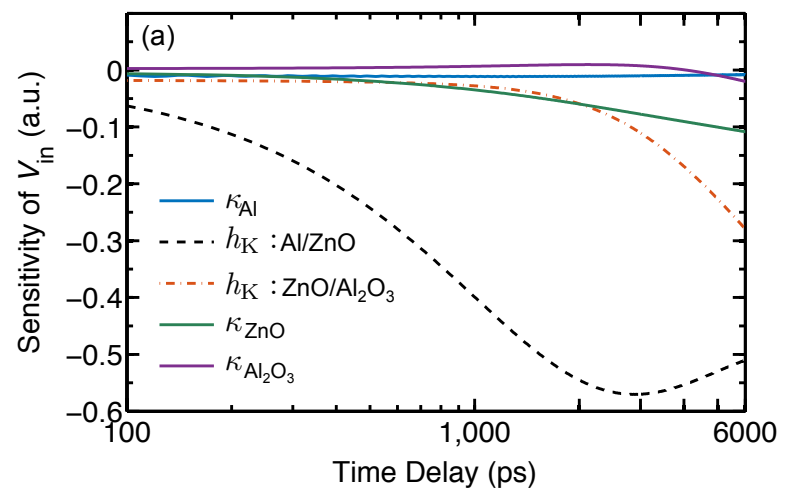

Figure 2.7: Sensitivities of the in-phase signal (for an $\mathrm{ALD}$ grown $\mathrm{ZnO}$ thin film) to the thermal boundary conductances at $\mathrm{Al} / \mathrm{ZnO}$ and $\mathrm{ZnO} / \mathrm{Al}_{2} \mathrm{O}_{3}$ interfaces and thermal conductivities of $\mathrm{Al}, \mathrm{ZnO}$ and $\mathrm{Al}_{2} \mathrm{O}_{3}$.

To determine the back and front side conductance of $\mathrm{Al} / \mathrm{ZnO} / \mathrm{Al}_{2} \mathrm{O}_{3}$ system, a combination of the in-phase response and the ratio of the in-phase to out-of-phase responses is used over various pump-probe time delays, due to relative sensitivities to the thermophysical properties of interest in this system at different time delay regimes. The sensitivity of the in-phase signal to various thermal properties is defined by,

$$
S_{a}=\frac{\partial \ln \left(-V_{\text {in }}\right)}{\partial \ln (a)}
$$

where $a$ is the thermophysical parameter of interest and $V_{\text {in }}$ is directly proportional to the response of the thermoreflectance signal recorded by the lock-in amplifier. Figure 2.7 shows the sensitivites of $V_{\text {in }}$ to the thermophysical properties of interest in the $\mathrm{ZnO}$ control sample at $300 \mathrm{~K}$. The sensitivity to $h_{\mathrm{K}}$ for the $\mathrm{Al} / \mathrm{ZnO}$ interface is relatively large and very dynamic for the first nanosecond time delay. In this time frame, the sensitivities of the other parameters are minimal and therefore will not affect the thermal response of the control sample. The in-phase signal is fit with the thermal model by iterating $h_{\mathrm{K}}$ for the 

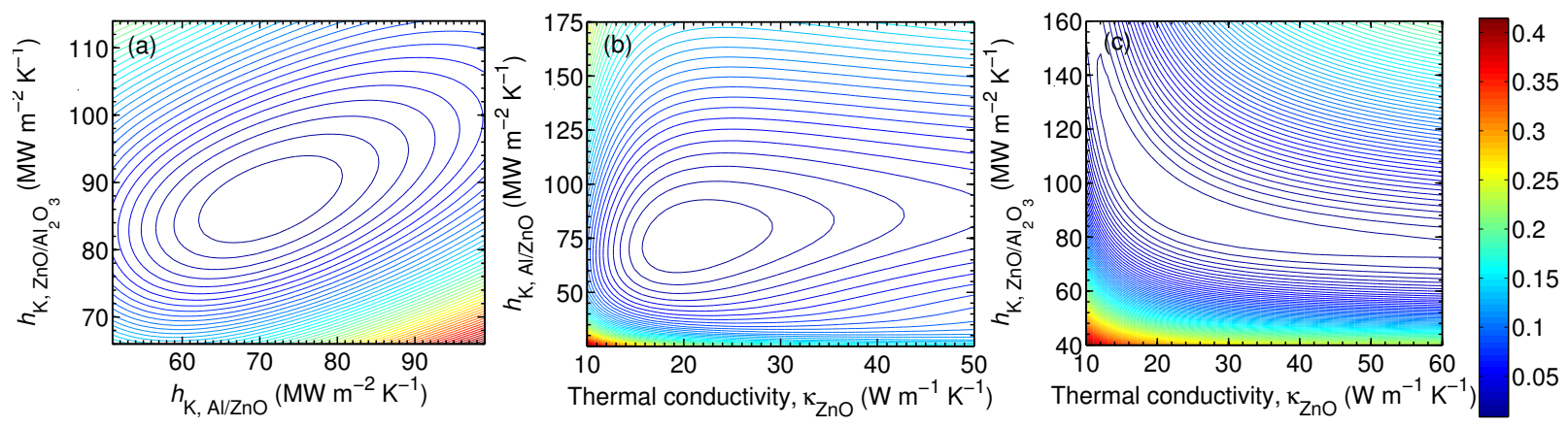

Figure 2.8: Sensitivity contour plots showing the interrelationship between (a) $h_{\mathrm{K}}$ at $\mathrm{ZnO} / \mathrm{Al}_{2} \mathrm{O}_{3}$ interface and $h_{\mathrm{K}}$ at $\mathrm{Al} / \mathrm{ZnO}$ interface, (b) $h_{\mathrm{K}}$ at $\mathrm{Al} / \mathrm{ZnO}$ interface and $\kappa_{\mathrm{ZnO}}$ and (c) $h_{\mathrm{K}}$ at $\mathrm{ZnO} / \mathrm{Al}_{2} \mathrm{O}_{3}$ and $\kappa_{\mathrm{ZnO}}$ in the three-layer thermal model for the purely ALD grown $\mathrm{ZnO}$ thin film at $8.8 \mathrm{MHz}$ pump modulation frequency and room temperature.

$\mathrm{Al} / \mathrm{ZnO}$ interface and all the other parameters are held constant for $1 \mathrm{~ns}$ time delay. It should be noted that since fitting the in-phase response of the TDTR signal requires scaling the model to the data at a fixed delay time (which we choose as $100 \mathrm{ps}$ ), the model is completely insensitive to thermophysical properties that have flat sensitivities in the time domain, further enhancing the accuracy in determining $h_{\mathrm{K}}$ over the specified time delay. Similarly, the $h_{\mathrm{K}}$ for the $\mathrm{ZnO} / \mathrm{Al}_{2} \mathrm{O}_{3}$ interface is determined by fitting the in-phase signal in the range of 2-5 ns while using the $h_{\mathrm{K}}$ for the $\mathrm{Al} / \mathrm{ZnO}$ interface determined from the first 1 ns time delay fit.

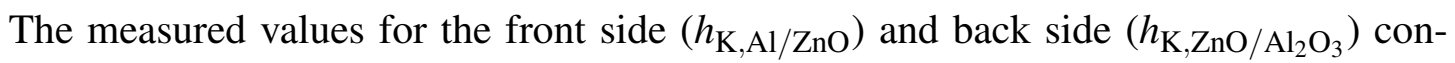
ductances at room temperature using this aforementioned procedure of fitting using the in-phase signal are $75 \pm 11$ and $85 \pm 13 \mathrm{MW} \mathrm{m}^{-2} \mathrm{~K}^{-1}$, respectively. These conductances present a major source of uncertainty in the measured values of thermal conductivities for the hybrid films as will be discussed later. Therefore, to provide further confidence to the independently measured values of these conductances from the control sample (using the in-phase signal), three contour plots are shown that demonstrate the deviation of the three-layer model from the ratio ( $\left.-V_{\text {in }} / V_{\text {out }}\right)$ as a function of $h_{\mathrm{K}, \mathrm{Al} / \mathrm{ZnO}}$ and $h_{\mathrm{K}, \mathrm{ZnO} / \mathrm{Al}_{2} \mathrm{O}_{3}}$ with $\kappa_{\mathrm{ZnO}}$ fixed to $42 \mathrm{~W} \mathrm{~m}^{-1} \mathrm{~K}^{-1}$ (as measured for a purely ALD grown $\mathrm{ZnO}$ thin film in Ref. [91]), deviation as a function of $h_{\mathrm{K}, \mathrm{Al} / \mathrm{ZnO}}$ and $\kappa_{\mathrm{ZnO}}$ with $h_{\mathrm{K}, \mathrm{ZnO} / \mathrm{Al}_{2} \mathrm{O}_{3}}$ fixed, and finally 
deviation as a function of $\kappa_{\mathrm{ZnO}}$ and $h_{\mathrm{K}, \mathrm{ZnO} / \mathrm{Al}_{2} \mathrm{O}_{3}}$ with $h_{\mathrm{K}, \mathrm{Al} / \mathrm{ZnO}}$ fixed (Fig. 2.8). These sensitivity contour plots represent the mean square deviation of the three-layer model to the ratio of the in-phase and out-of-phase signals with the various combinations of input parameters in the model [92]. As shown in Fig. 2.8, with the thermal conductivity of $\mathrm{ZnO}$ set to $42 \mathrm{~W} \mathrm{~m}^{-1} \mathrm{~K}^{-1}$, the front side and back side conductances predicted from the three-layer model agree with the values measured from $V_{\text {in }}$ by fitting the signal for the first nanosecond. However, as shown in Fig. $2.8 \mathrm{~b}$ and $\mathrm{c}$, a large range of values for $\kappa_{\mathrm{ZnO}}$ can produce the best-fit in the least squares sense. Therefore, it is not possible to accurately determine $\kappa_{\mathrm{ZnO}}$ from our analysis of the experimental data. This is due to the fact that the ALDgrown $\mathrm{ZnO}$ thin film is very thermally conductive and the analytical model is not sensitive to $\kappa_{\mathrm{ZnO}}$. Although, the $\kappa_{\mathrm{ZnO}}$ can not be accurately measured, the values for the front side and back side conductances that produce the best-fit of the model to the ratio of the TDTR signals agree with the measurements of these conductances (from $V_{\text {in }}$ by fitting at various time delays), thus supporting the use of the measured values of front side and back side conductances as input parameters in the model that is used to measure the thermal conductivities of the hybrid SL films. Note, for the hybrid SLs with high interface densities (and therefore low thermal conductivities), the three-layer model losses sensitivity to the front side and back side conductances, which presents comparatively low uncertainties as the thermal conductivity of the hybrid films decrease.

As is clear from the above discussions, one of the significant challenges to accurately describe thermal transport in materials from TDTR measurements is to effectively separate the contributions from $\kappa$ and $h_{\mathrm{K}}$ to the measured thermoreflectance signal. It should also be noted that measuring the $h_{\mathrm{K}}$ across low thermal conductivity materials in a TDTR experiment is another challenge, which arises due to the fact that the sensitivity to the TDTR measurement is mostly dominated by the bigger resistance (which is the low thermal conductivities of the materials) [93]. In this regard, in Chapters 4 and 5, the high interface densities in the SL structures will be utilized by analyzing the results in terms of a thermal circuit model to back-out the mean conductances across these interfaces and shed more 
light on the characteristic internal scattering mechanisms in the various SL structures.

\subsubsection{The two-temperature model}

The heat diffusion equation represented in Eq. 2.19 is a valid description of heat flow as long as the local equilibrium is maintained between all energy carriers. In other words, a single temperature can define the occupation of thermal excitations on time and length scales that are comparable to the mean free paths of the thermal excitations. However, during strong e-p non-equilibrium that is typical in short-pulse irradiation on a metal surfaces for the first few picoseconds after pulse absorption, the applicability of Eq. 2.19 breaks down and non-Fourier models have to be applied to accurately describe the heat flow [9496]. Therefore, to understand the e-p dynamics occurring in the short-time regime (1-10 ps after pulse absorption) the TTM is utilized to analyze the experimental data [56]. In the TTM, the electronic and phononic systems are described by two separate temperatures. The respective time evolution of the electron and lattice temperatures are given by,

$$
\begin{array}{r}
C_{e}\left(T_{\mathrm{e}}\right) \frac{\partial T_{\mathrm{e}}}{\partial t}=\nabla \cdot\left(\kappa_{e} \nabla T_{\mathrm{e}}\right)-G\left(T_{\mathrm{e}}-T_{\mathrm{p}}\right)+S(t), \\
C_{p}\left(T_{\mathrm{p}}\right) \frac{\partial T_{\mathrm{p}}}{\partial t}=\nabla \cdot\left(\kappa_{p} \nabla T_{p}\right)+G\left(T_{\mathrm{e}}-T_{\mathrm{p}}\right)
\end{array}
$$

where $G$ is the volumetric rate of energy transfer between the two states [ $\mathrm{W} \mathrm{m}^{-3} \mathrm{~K}^{-1}$ ], $C_{e}$ and $C_{p}$ are the heat capacities of the electrons and phonons, respectively. Similarly, $\kappa_{e}$ and $\kappa_{p}$ are the thermal conductivities of the electrons and phonons, respectively, and $S(t)$ is the source term that is modified to account for the delayed relaxation in the electronic distribution [97]. Note, in the limit of $T_{\mathrm{e}}=T_{\mathrm{p}}$, Eq. 2.21] reduces to Eq. 2.19.

To implement the TTM, a thermoreflectance model is needed to relate the change in reflectivity to the excursions in temperatures. In this context, the validity of Eq. 2.18 breaks down at elevated temperatures (corresponding to high laser fluences) as the relation between temperature and reflectivity due to intraband transitions is highly nonlinear. Smith and Norris [88] developed a thermoreflectance model that expands the temperature range 
applicable to measure thermophysical properties by exploiting the relationship between the metal's reflectivity and the change in the dielectric function due to changes in electron and phonon temperatures. However, the complex dielectric function combines the effects due to interband transitions (free electrons) and intraband transitions (bound electrons), $\hat{\varepsilon}=\hat{\varepsilon}_{\text {intra }}+\hat{\varepsilon}_{\text {inter. }}$ Since we are examining Au with $800 \mathrm{~nm}$ pulses $(1.55 \mathrm{eV})$, only the intraband transitions need to be considered as the lowest energy $d$-band to available $s$-band transition is very large for $\mathrm{Au}(2.4 \mathrm{eV})$.

The intraband part, $\hat{\varepsilon}_{\text {intra }}$, is described by the well-known Drude model [98, 99],

$$
\hat{\varepsilon}_{\text {intra }}=1-\frac{\omega_{\mathrm{p}}^{2}}{\omega\left(\omega+i \tau_{\mathrm{f}}^{-1}\right)}
$$

where $\omega$ is the angular frequency of the absorbed radiation, $\omega_{\mathrm{p}}$ is the plasma angular frequency of the film and $\tau_{\mathrm{f}}^{-1}$ is the scattering rate of the free electrons undergoing intraband transitions. The temperature dependence in Eq. 2.22 comes from the scattering rate of these free electrons and can be estimated through the Matthiessen's rule [93], $\tau_{\mathrm{f}}^{-1}=A_{\mathrm{ee}} T_{\mathrm{ee}}{ }^{2}+B_{\mathrm{ep}} T_{\mathrm{p}}$, where $A_{\mathrm{ee}}$ and $B_{\mathrm{ep}}$ are constant coefficients relating to the temperature dependencies of e-e and e-p collisional frequencies, respectively. The thermoreflectance model requires the proper knowledge of these scattering coefficients and in an earlier work from our group [100], it has been shown that by replacing $G$ in the TTM with $G_{\text {eff }}=\left(\pi^{2} m v_{\mathrm{s}}^{2} n_{\mathrm{e}}\right) / 6\left[A_{\mathrm{ee}}\left(T_{\mathrm{e}}+T_{\mathrm{p}}\right)+B_{\mathrm{ep}}\right]$ (that is derived in Ref. [101] $)$ and fitting for the scattering coefficients in the regime of low electron perturbation, the values for $A_{\text {ee }}$ and $B_{\text {ep }}$ can be effectively predicted. Using this procedure, for the thin Au film studied in Chapter 3, the predicted scattering coefficients were $A_{\mathrm{ee}}=1.1 \times 10^{7} \mathrm{~K}^{-2} \mathrm{~s}^{-1}$ and $B_{\mathrm{ep}}=1.3 \times 10^{-11}$ $\mathrm{K}^{-1}$, in excellent agreement with the low temperature resistivity data [102] and literature values [103].

The reflectivity of a bulk material at the air (vaccum)/film interface is given by,

$$
R=\frac{\left(n_{1}-1\right)^{2}+n_{2}^{2}}{\left(n_{1}+1\right)^{2}+n_{2}^{2}}
$$


where $n_{1}$ and $n_{2}$ are the real (refractive index) and imaginary (extinction coefficient) parts of the complex index of refraction. The intraband part of the optical dielectric function, $\hat{\varepsilon}_{\text {intra }}=\varepsilon_{1}+i \varepsilon_{2}$, is further related to the refractive index and extinction coefficient through, [99]

$$
n_{1}=\frac{1}{\sqrt{2}}\left[\left(\varepsilon_{1}^{2}+\varepsilon_{2}^{2}\right)^{1 / 2}+\varepsilon_{1}\right]^{1 / 2}
$$

and

$$
n_{2}=\frac{1}{\sqrt{2}}\left[\left(\varepsilon_{1}^{2}+\varepsilon_{2}^{2}\right)^{1 / 2}-\varepsilon_{1}\right]^{1 / 2}
$$

Eq. 2.23 does not account for multiple reflections and absorptions at the film/substrate interface for thin films that are in the order of the optical penetration depth. As the film/substrate systems studied in this thesis are optically thin at $800 \mathrm{~nm}$, thin film optics will be used to calculate the reflectivity where the incident medium is air,

$$
R_{\mathrm{f}}=r^{*} r
$$

where

$$
r=\frac{\left(m_{11}+\hat{n}_{\mathrm{s}} m_{12}\right)-\left(m_{21}+\hat{n}_{\mathrm{s}} m_{22}\right)}{\left(m_{11}+\hat{n}_{\mathrm{s}} m_{12}\right)+\left(m_{21}+\hat{n}_{\mathrm{s}} m_{22}\right)}
$$

with $\hat{n}_{\mathrm{s}}$ being the complex index of refraction of the substrate $\left(\hat{n}_{\mathrm{s}}=n_{1, \mathrm{~s}}+i n_{2, \mathrm{~s}}\right)$ and $r^{*}$ is the complex conjugate of Eq. 2.26, $m_{\mathrm{ij}}$ is the component of the characteristic thin film matrix defined as

$$
m_{\mathrm{ij}}=\left[\begin{array}{cc}
\cos \delta & -\frac{i}{\hat{n}_{\mathrm{f}}} \sin \delta \\
-i \hat{n}_{\mathrm{f}} \sin \delta & \cos \delta
\end{array}\right]
$$

where $\delta=\omega d \hat{n}_{\mathrm{f}} / c$ and $c$ is the speed of light and $d$ is the film thickness. Once Eq. 2.26 is determined, the final change in reflectivity due to temperature changes of the film/substrate 


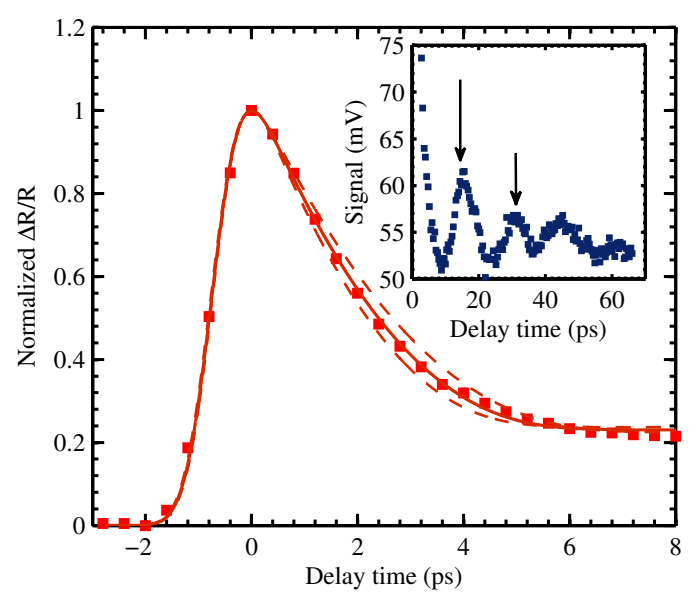

Figure 2.9: TDTR signal on $26 \mathrm{~nm} \mathrm{Au} / \mathrm{Si}$ sample at an absorbed laser fluence of $\mathrm{F}=3.52 \mathrm{~J}$ $\mathrm{m}^{-2}$ and corresponding best fit using the modified TTM with a nonlinear thermoreflectance model. (inset) Picosecond acoustic response of the film used to measure the thickness.

system is given by,

$$
\frac{\Delta R}{R}=\frac{R_{\mathrm{f}}\left(T_{\mathrm{e}, \mathrm{eff}}\right)-R_{\mathrm{f}}\left(T_{0}\right)}{R_{\mathrm{f}}\left(T_{0}\right)}
$$

where $T_{0}$ is the ambient temperature. An example of the fit with the TDTR data for $\sim 26$ $\mathrm{nm}$ Au film deposited on a Si substrate is shown in Fig. 2.9. The data is normalized by the maximum magnitude of the signal from the lock-in amplifier. The predicted maximum effective electron temperature in this data is $1,750 \pm 120 \mathrm{~K}$ with a $G_{\text {eff }}=2.90 \pm 0.2 \times 16 \mathrm{~W}$ $\mathrm{m}^{-3} \mathrm{~K}^{-1}$, which is in good agreement with previous literature [97, 104]. The dotted lines in Fig. 2.9 represent the uncertainties involved with the fitting procedure (such as uncertainties due to error in thickness measurement). The inset shows the picosecond acoustics response of the film, which is used to measure the thickness of the film. The technique relies on the oscillatory change in the thermoreflectance signal due to the propagation of strain wave caused by the sudden heating of the film. The round-trip time of the echo can be observed in the oscillatory signal (marked by the two arrows) and the thickness can be determined by correlating it to the longitudinal speed of sound in the gold film.

The TTM and the thermoreflectance model described above will be applied to analyze 
the early pump-probe delay time data in the next chapter to determine $G_{\text {eff }}$ for various samples with a thin Au layer on top. 


\section{Chapter 3}

\section{Mechanisms of electron-phonon}

\section{coupling at interfaces}

This chapter investigates the electron energy transfer processes occurring at and near the film-substrate interfaces, and how these processes affect thermal transport at different time scales after short pulsed laser heating. The coupling between the electronic and the vibrational states is increased by more than a factor of five with the inclusion of an $\sim 3$ $\mathrm{nm}$ Ti adhesion layer between the Au film and the non-metal substrate. Furthermore, an increase in the rate of relaxation of the electron system is shown with increasing electron and lattice temperatures induced by the laser power and this is attributed to enhanced electron-electron scattering, a transport channel that becomes more pronounced with increased electron temperatures. The inclusion of the Ti layer also results in a linear dependence of the electron-phonon relaxation rate with temperature which is attributed to the coupling of electrons at and near the Ti/substrate interface. This enhanced electronphonon coupling due to electron-interface scattering is shown to have negligible influence on the thermal boundary conductances between the $\mathrm{Au} / \mathrm{Ti}$ and the substrates at longer time scales when the electrons and phonons in the metal have equilibrated. These results suggest that only during highly nonequilibrium conditions between the electrons and phonons $\left(T_{\mathrm{e}} \gg T_{\mathrm{p}}\right)$ does electron-phonon scattering at an interface contribute to thermal boundary 
conductance. A large portion of the chapter appeared in "Mechanisms of nonequilibrium electron-phonon coupling and thermal conductance at interfaces" in the Journal of Applied Physics [105].

\subsection{Background}

Immediately after excitation with an ultrashort laser pulse, the electron gas in a metal can be heated to several thousand degrees above the lattice temperature due to the large differences in their specific heats. Pump-probe thermoreflectance measurements allow the capability to examine the Fermi relaxation dynamics, electron-phonon (e-p) relaxation as well as phonon-phonon (p-p) thermalization processes by relating the change in reflectivity of the sample surface to various rate-relaxation models. For example, the e-p coupling factor, $G$, for a thin Au film on a dielectric substrate can be determined by fitting the initial rise and the fast transient decay of the TDTR signal in the first few picoseconds (as shown in Fig. 2.9] to the TTM [56].

Typically, heat transport across metal/non-metal interfaces is found to be dominated by phonon-phonon scattering processes [7, 22]. However, there exist several experimental results that can not be predicted by theoretical models that are solely based on phonon mediated transport such as the well known DMM and the AMM [19, 22, 25]. As mentioned in Chapter 1, many theories have attempted to explain these results via the e-p interfacial coupling mechanisms. Previous works from our group (Ref. [36]) have shown that during conditions of strong e-p and e-e nonequilibrium, this electron-interface coupling mechanism could in fact be contributing to the TBC at those time regimes. However, this mechanism of interfacial transport has been relatively unexplored, and fundamental questions regarding how substrate and interfacial properties affect this heat transport mechanism are unknown.

In light of the discussions in the previous paragraphs, the effective e-p coupling factor, $G_{\text {eff }}$, is measured for Au films on various non-metal substrates with and without a very thin 
$(\sim 3 \mathrm{~nm})$ Ti adhesion layer across a wide range of electron temperatures $\left(T_{\mathrm{e}} \sim 400-2000\right.$ $\mathrm{K})$. The thin $\mathrm{Ti}$ adhesion layer is deposited to not only enhance the bonding between the metal film and the dielectric substrate, but also to provide a strong channel for e-p energy exchange near the interface relative to the weak e-p coupling characteristic of Au.

\subsection{Experimental considerations}

Nominally $20 \mathrm{~nm}$ of gold films with and without a titanium adhesion layer were evaporated onto crystalline silicon, crystalline sapphire and fused silica using electron-beam evaporation. The thicknesses of the heterostructures were measured via the X-ray reflectivity (XRR) technique by our colleagues at Sandia National Labratories (Drs. Mark A. Rodriguez and Jon Ihlefeld), which was performed using a Scintag PAD X diffractometer equipped with a sealed-tube $\mathrm{Cu}$ anode (goniometer radius $=240 \mathrm{~mm}$ ). Generator settings were $40 \mathrm{kV}$ and $30 \mathrm{~mA}$. Monochromatic $\mathrm{Cu} \mathrm{Ka}(0.15406 \mathrm{~nm})$ radiation was generated through the use of an incident beam mirror optic. The mirror exit aperture was set to a height of 50 microns to ensure minimal beam loss at low angles (i.e. $<0.5^{\circ} 2 \theta$ ) so as to assure accurate critical edge determination. The diffraction system employed a Peltier-cooled Ge solid-state detector with a $0.2^{\circ}$ receiving slit. XRR data were fit using the software program Parratt32 (Version 1.5.2) employing a two-layer (Au/Ti/substrate) model. The measured thicknesses are tabulated in Table 3.1. Atomic force microscopy study conducted on all the samples exhibited high quality smooth surfaces with roughnesses less than $1 \mathrm{~nm}$.

For the TDTR measurements, the laser spot sizes were focused on to the sample surface through a $10 \times$ objective to $1 / e^{2}$ radii values of 7.0 and $6.0 \mu \mathrm{m}$ for pump and probe beams, respectively. The average probe power in these experiments was fixed at $9 \mathrm{~mW}$ whereas the average pump power was adjusted between experiments from $5 \mathrm{~mW}$ to $60 \mathrm{~mW}$ to probe the effects of increasing e-p nonequilibrium. The absorption of the pump pulses by the sample surface leads to a rapid increase in the internal energy of the electron system, which then transfers its energy to the lattice vibrations. This provides a unique path forward to directly 
Table 3.1: Parameters used in the thermoreflectance model for the $\mathrm{Au} / \mathrm{Ti} / \mathrm{Si}, \mathrm{Au} / \mathrm{Ti} / \mathrm{Al}_{2} \mathrm{O}_{3}$ and $\mathrm{Au} / \mathrm{Ti} / \mathrm{SiO}_{2}$ structures. $\gamma_{e}$ is the electronic heat capacity coefficient in the TTM. The thicknesses of $\mathrm{Au}$ and Ti bi-layers were measured by X-ray reflectivity measurements.

\begin{tabular}{ccc}
\hline \hline & $\mathrm{Au}$ & $\mathrm{Ti}$ \\
\hline$C_{p}\left(\times 10^{6} \mathrm{~J} \mathrm{~m}^{-3} \mathrm{~K}^{-1}\right)$ & 2.49 (Ref. [106]) & 3.01 (Ref. [107]) \\
$\gamma_{e}\left(\mathrm{~J} \mathrm{~m}^{-3} \mathrm{~K}^{-2}\right)$ & 62.9 (Ref. [58]) & 328.9 (Ref. [58]) \\
$d(\mathrm{~nm})$ on $\mathrm{Si}_{\text {substrate }}$ & $16.1 \pm 0.2$ & $3.1 \pm 0.2$ \\
$d(\mathrm{~nm})$ on $\mathrm{Al}_{2} \mathrm{O}_{3}$ substrate & $16.2 \pm 0.6$ & $2.7 \pm 0.2$ \\
$d(\mathrm{~nm})$ on $\mathrm{SiO}_{2}$ substrate & $15.7 \pm 0.7$ & $2.8 \pm 0.7$ \\
\hline \hline
\end{tabular}

measure how electrons in various states of nonequilibrium interact with the surrounding vibrational enviornment in metal nanosystems. The change in the optical properties created due to this thermal relaxation process is measured with the reflected probe beam at the frequency with which the pump beam is modulated in the experiment $(8.8 \mathrm{MHz})$.

The temperature dynamics underlying the measured thermoreflectance data were determined using the TTM approach, which takes into account the delay in the thermalization process of the electronic system. The ballistic nature of the "hot" electrons in the thin films creates a homogeneously heated thin film which simplifies the modified TTM by eliminating the electron and phonon diffusion terms during e-p equilibration. Note, the parabolic two-step radiation model (Eq. 2.21) that is used to analyze the data in this work assumes infinite speeds of energy propagation [96], which for the thin film samples studied in this chapter is a valid approximation since there are no spatial gradients in the nonthermal distribution of the electrons. However, for thicker samples that are greater than the ballistic penetration depth of the electrons in Au, the hyperbolic two-step radiation heating model (as explained in Ref. [96]) would be the more appropriate thermal model to analyze the TDTR data.

To determine the initial energy distribution in the multilayer films, the transfer matrix method [108] is used to calculate the light absorption as a function of space in the $\mathrm{Au} / \mathrm{Ti}$ bi-layers. A mixing rule based on the percentage of light absorbed by the electron system 
in the bi-layers is used to determine the material parameters in TTM. Table 3.1 lists the thicknesses and essential material parameters used as inputs to the TTM. It should be noted that even though $\mathrm{Ti}$ is not a free electron metal, the free electron approximation for electron heat capacity agrees well with heat capacity calculated using an ab initio-dervied electron density of states [58]. It should also be noted that it is not possible to spatially discretize the thin films due to the fact that the thicknesses are less than the mean free path of e-p relaxation in the respective bilayers.

The amount of laser energy absorbed by the electrons in the sample is calculated according to the thin film optics approach explained in Refs. [109, 110] and the Drude-based thermoreflectance model is used to analyze the data (as explained in the earlier chapter). Precaution is taken to make sure that the absorbed fluence does not increase the conduction band number density due to $d$-band excitations. This is because the model only takes into account the change in reflectivity due to intraband transitions [111]. The probe energy is well below the interband transition threshold for $\mathrm{Au}(2.4 \mathrm{eV})$ supporting the use of the aforementioned intraband thermoreflectance model. Even at the maximum absorbed laser fluence, the conduction band number density will only be perturbed by $<2 \%$ [112]. Note that the effective electron temperatures in the predictions from the TTM do not exceed 3,000 K. Above this temperature, the conduction band number density is changed due to $d$-band transitions that cause a change in the chemical potential and occupied density of states, rendering the thermoreflectance model ineffective [58, 113].

The magnitude of the thermoreflectance signal is analyzed using the values of $A_{e e}$ and $B_{e p}$ determined for $\mathrm{Au} / \mathrm{Ti} / \mathrm{Si}, \mathrm{Au} / \mathrm{Ti} / \mathrm{Al}_{2} \mathrm{O}_{3}$ and $\mathrm{Au} / \mathrm{Ti} / \mathrm{SiO}_{2}$ systems. The thermoreflectance model is fit to the experimental data by normalizing the peak electron temperature to the peak in the reflectance signal while iterating $G_{\text {eff }}$ until the minimum error between the model and the data is produced. Figure 3.1 compares the TDTR data and TTM fits for a $20 \mathrm{~nm}$ Au film on a fused silica substrate (red square) and a $15.7 \mathrm{~nm} \mathrm{Au} / 2.8 \mathrm{~nm}$ Ti on fused silica substrate (blue circle). The fast transient decay in the signal for $\mathrm{Au} / \mathrm{Ti} /$ fused silica shows that the inclusion of the Ti adhesion layer significantly decreases the electron 


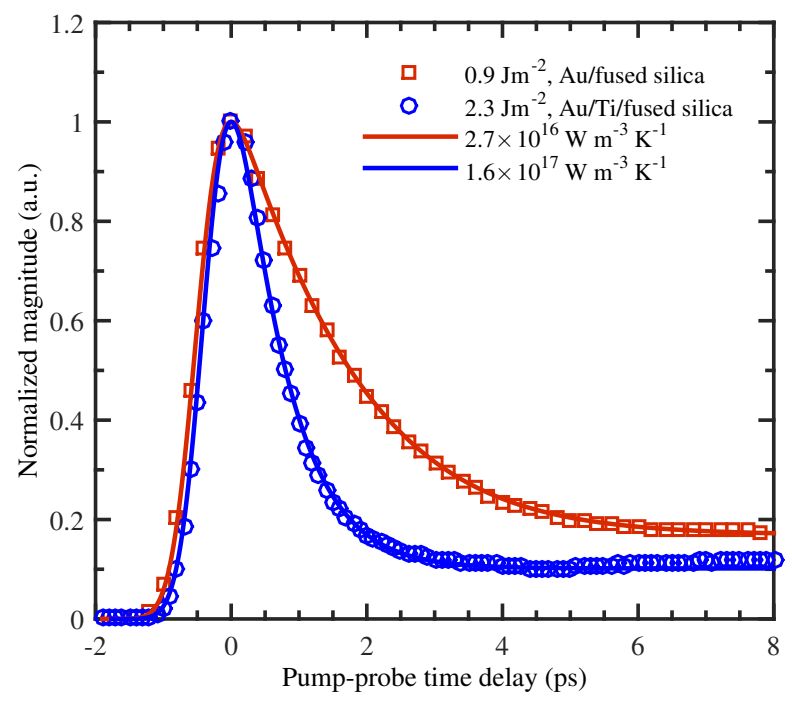

Figure 3.1: TDTR data on Au/fused silica (red square) and Au/Ti/fused silica (blue circle) samples at room temperature and corresponding best-fits using the TTM with a nonlinear thermoreflectance model. The data is normalized by the maximum magnitude of the signal from the lock-in amplifier.

relaxation time and causes an increase in the measured $G_{\text {eff }}$ values.

\subsection{Ballistic transport and electron scattering at the inter- face}

Figure 3.2 shows the measured values of $G_{\text {eff }}$ as a function of total temperature of the electronic and vibrational states in Au films with Ti adhesion layers on three substrates (sapphire, silicon and fused silica). The total temperature is defined as the sum of the maximum lattice and electron temperatures predicted via the TTM analysis. Due to the relatively lower thermal effusivities of the fused silica and sapphire substrates compared to that of silicon, there is an additional temperature rise in the metal which we refer to as DC heating, $\Delta T_{\mathrm{DC}}$. This temperature rise of the metal bi-layers can be estimated through the expression [114],

$$
\Delta T_{\mathrm{DC}}=\frac{(1-R) \dot{q}}{\kappa\left(2 \pi \omega_{0}^{2}+2 \pi \omega_{1}^{2}\right)^{1 / 2}}
$$


Here $\dot{q}$ is the incident laser power, $R$ is the reflectivity, $\kappa$ is the thermal conductivity of the substrate, and $\omega_{0}$ and $\omega_{1}$ are pump and probe radii, respectively. Not accounting for this DC heating results in an under prediction of the rate of e-p relaxation [100].

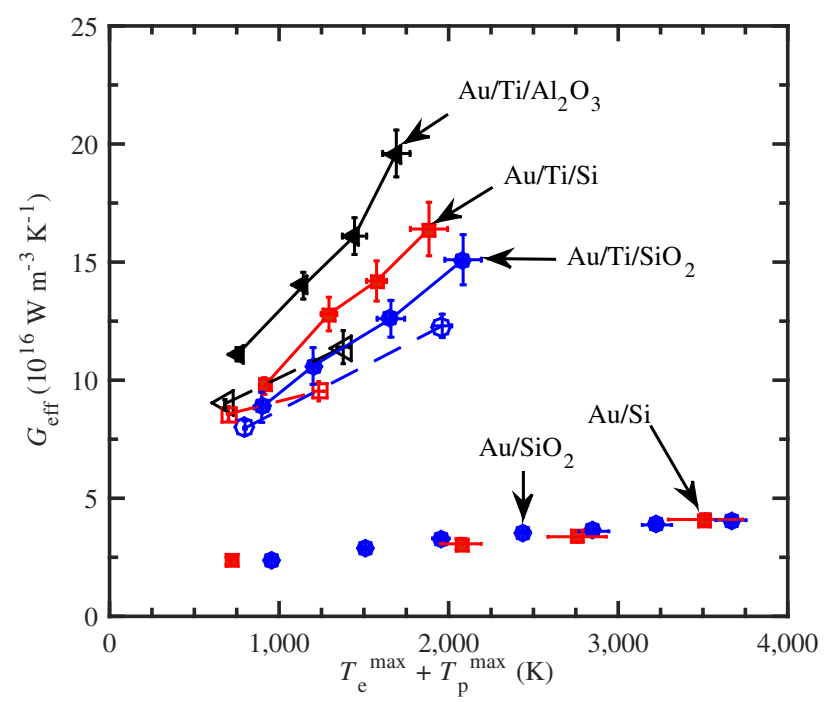

Figure 3.2: $G_{\text {eff }}$ as a function of maximum effective electron temperature plus maximum phonon temperature from DC laser heating for $\mathrm{Au} / \mathrm{Ti} /$ fused silica (blue circle), Au/Ti/Si (red square) and $\mathrm{Au} / \mathrm{Ti} / \mathrm{sapphire} \mathrm{(black} \mathrm{triangle).} \mathrm{For} \mathrm{comparison,} \mathrm{we} \mathrm{have} \mathrm{also} \mathrm{plotted}$ $G_{\text {eff }}$ values for $\mathrm{Au} / \mathrm{Si}$ and $\mathrm{Au} /$ fused silica. The hollow symbols represent data for Au films with a thickness of $40 \mathrm{~nm}$ with Ti adhesion layer on different substrates. After accounting for $\Delta T_{\mathrm{DC}}$ in $T_{\mathrm{p}}{ }^{\max }$ (due to local heating of the Au lattice), TTM fits to the Au/fused silica data results in similar temperature trends between the determined $G_{\text {eff }}$ for Au/fused silica and $\mathrm{Au} / \mathrm{Si}$ systems. However, we observed a much larger enhancement in $G_{\text {eff }}$ for systems with the inclusion of the Ti layer.

For comparison, Fig. 3.2 also plots the $G_{\text {eff }}$ values for samples without the Ti layer. The agreement between the measured $G_{\text {eff }}$ for these samples suggests that the mechanisms driving e-p relaxation are intrinsic to $\mathrm{Au}$ films and independent of the $\mathrm{Si}$ and fused silica substrates along with the interfacial region between the Au and the substrate. However, with the inclusion of the thin Ti layer, the dependency of $G_{\text {eff }}$ on the substrate becomes prominent and the $G_{\text {eff }}$ increases by as much as five fold in the electron temperature range measured in the experiments, the implications of which will be discussed in more detail. The hollow symbols represent data for thicker Au films (40 nm) with Ti adhesion layer. 
Although the thickness of the Au films for these samples are still below the ballistic length of Au electrons, the measured $G_{\text {eff }}$ values are lower compared to the thinner Au films with Ti adhesion layer, the reason for which will also be discussed in the following paragraphs. Furthermore, the substrate dependence for these samples is not as pronounced as for the thinner films.

For the Au films without $\mathrm{Ti}$ adhesion layers, the increase in the rate of relaxation due to an increase in laser fluence can be understood as a consequence of increased phase space of the electrons taking part in the scattering mechanisms [115, 116]. The absorption of the pump pulse incident on the metal surface creates an electron-hole pair distribution near a narrow region $\left(\sim 1.5 k_{\mathrm{B}} T_{\mathrm{e}}\right)$ around the Fermi surface. The Pauli exclusion principle dictates that only the electrons in this region are allowed to participate in the energy relaxation process through collisions. Higher fluences leading to higher $T_{\mathrm{e}, \text { eff }}$ cause more electrons to take part in the relaxation process that ultimately increases $G_{\text {eff }}$.

The difference between the measured $G_{\text {eff }}$ values in the Au films on different substrates with the inclusion of the Ti layer suggests that scattering of the excited Au electrons in the interfacial region ( $\mathrm{Ti} /$ substrate) plays a role in the enhancement of e-p coupling in these nanosystems. However, other scattering mechanisms that could be working in tandem to augment heat flow in these systems cannot be ruled out. For example, the electronic thermal conductance between the Au and Ti layer, $h_{\mathrm{ee}}$, and the electronic thermal conductivity and e-p coupling in each layer in adjunction to metal-electron/interface energy transfer, $h_{\mathrm{ei}}$, could govern thermal transport at these short time scales. Experimental values of $h_{\mathrm{ee}}$ on various metal-metal interfaces have shown that the conductances are an order of magnitude larger than the phonon mediated conductances [14, 117]. Theoretically, due to this above argument, $h_{\mathrm{ee}}$ can be safely neglected as other conductances control the thermal transport in these systems. This assertion will be experimentally supported later in this section.

At room temperature, the characteristic e-p relaxation length scale is $\sqrt{\kappa_{e} / G}$, where $\kappa_{e}$ is the electronic thermal conductivity. Using a value of $320 \mathrm{~W} \mathrm{~m}^{-1} \mathrm{~K}^{-1}$ for the electronic thermal conductivity and a value of $3 \times 10^{16} \mathrm{~W} \mathrm{~m}^{-3} \mathrm{~K}^{-1}$ for e-p coupling in Au yields an 
e-p mean free path of $\approx 100 \mathrm{~nm}$. Similarly, for Ti, using $\kappa_{e}=8.2 \mathrm{~W} \mathrm{~m}^{-2} \mathrm{~K}^{-1}$ determined from electrical resistivity measurements $[118]$ and $G=1.3 \times 10^{18} \mathrm{~W} \mathrm{~m}^{-3} \mathrm{~K}^{-1}$ (Ref. [58]), results in a mean free path of $\sim 3 \mathrm{~nm}$. The thicknesses of the bi-layers used in this work (Table 3.1) are less than the e-p mean free paths for the respective metals. For these thin film limits, the effective conductance due to e-p coupling is $G d$. Due to the very high value of $G$ in $\mathrm{Ti}\left(G=1.3 \times 10^{18} \mathrm{~W} \mathrm{~m}^{-3} \mathrm{~K}^{-1}\right.$ at room temperature) [58], the effective e-p conductance in the Ti layer is approximately $3 \mathrm{GW} \mathrm{m}^{-2} \mathrm{~K}^{-1}$, a value much greater than the effective conductance due to the weak e-p coupling in the Au layer $\left(\approx 350 \mathrm{MW} \mathrm{m}{ }^{-2} \mathrm{~K}^{-1}\right.$ at low absorbed laser fluences and room temperature conditions). These arguments suggest that the main parameters affecting thermal transport in samples with the thin Ti layers for the short time scales considered in this work are $h_{\mathrm{ei}}$ and e-p coupling in the Au layer. The effective e-p conductances in each metal layer are added in series (e-p resistances add in parallel) and therefore with the increase of the Au thickness, the weak coupling in the Au layer decreases the value of the $G_{\text {eff }}$ measured for the $40 \mathrm{~nm}$ Au films. It is important to note that for a thermally thick Ti layer, most of the energy will be deposited in the Ti layer due to the strong e-p coupling that effectively couples all the energy from the electrons to the lattice vibrations.

The TTM model does not explicitly separate the contributions from the various scattering mechanisms associated with electron energy transfer in the $\mathrm{Ti}$ interfacial region: i.e., e-e scattering across the $\mathrm{Au} / \mathrm{Ti}$ interface, e-p coupling in the $\mathrm{Ti}$ and electron-metal/phonon non-metal energy transfer. Hence, these three conductive pathways are collectively referred to as electron-interface conductance, or $h_{\mathrm{ei}}$, as previously defined. Although $G_{\mathrm{eff}}$ has been previously measured for thin Au films [119] the TTM analysis can not be applied directly to Ti because of the complicated electronic band structure around the Fermi energy which renders the Drude-based thermoreflectance model inapplicable [111]. However, from Fig. 3.2. it is clear that if the metal film does not strongly adhere with the nonmetal substrate, the substrate dependence in the e-p coupling no longer exists. To further understand the various scattering mechanisms contributing to $h_{\mathrm{ei}}$, measurements are repeated for samples with 

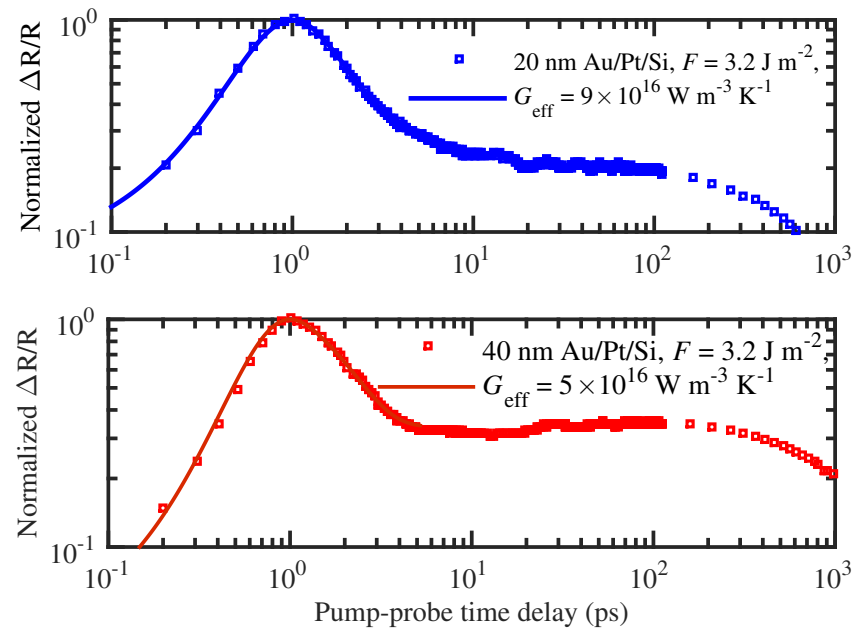

Figure 3.3: TDTR data on $20 \mathrm{~nm} \mathrm{Au} / \mathrm{Pt} / \mathrm{Si}$ and $40 \mathrm{~nm} \mathrm{Au} / \mathrm{Pt} / \mathrm{Si}$ samples with the corresponding fits using the TTM with a nonlinear thermoreflectance model for the initial few picoseconds. The thermoreflectance responses at longer pump-probe delay times are also shown where the periodic oscillations in the data for $10-100 \mathrm{ps}$ is representative of strain wave propagation.

a thin Pt layer instead of the Ti layer between the Au film and the nonmetal substrates. Pt is chosen due to the fact that the e-p coupling factor for Pt has been reported to be similar to the value for Ti [120] and also because Pt does not adhere strongly to the nonmetal substrates. Figure. 3.3 shows the thermoreflectance signals for a $40 \mathrm{~nm} \mathrm{Au} / \mathrm{Pt} / \mathrm{Si}$ and a $20 \mathrm{~nm}$ $\mathrm{Au} / \mathrm{Pt} / \mathrm{Si}$ along with the TTM fits. Similar to the thermoreflectance signal for Au films without the Ti layer, Au/Pt samples also showed the oscillatory signal for delay times of 10-100 ps. This observation is consistent with the fact that the metal films are not strongly adhered to the substrate as the weak bonding allows the strain wave to reflect off the metal/nonmetal interface without pronounced transmission (unlike the case with the $\mathrm{Au} / \mathrm{Ti}$ samples, which will be discussed in more detail in the next subsection). This leads to amplitude modulations in the film thickness due to longitudinal "breathing" of the metal film. The picosecond acoustics has been explained by Tas and Maris [115] from a macroscopic point of view as the propagation of an elastic wave that is launched in the sample due to a thermally induced stress from the laser pulse absorption. As the $20 \mathrm{~nm} \mathrm{Au} / \mathrm{Pt}$ and $20 \mathrm{~nm} \mathrm{Au} / \mathrm{Si}$ samples are 
heated homogeneously, the thermal stress created at the interface between the film and the substrate generates a thermal expansion of the film due to an elastic wave that reflects at the film/substrate interface.

Contrary to the increase in $G_{\text {eff }}$ as a function of electron temperature for samples with the $\mathrm{Ti}$ adhesion layer, the e-p coupling in the Au/Pt samples did not show a pronounced dependence on either laser fluence or the different substrates. The predicted $G_{\text {eff }}$ values ranged from $8-9 \times 10^{16} \mathrm{~W} \mathrm{~m}^{-3} \mathrm{~K}^{-1}$ to $4-5 \times 10^{16} \mathrm{~W} \mathrm{~m}^{-3} \mathrm{~K}^{-1}$ for $20 \mathrm{~nm}$ and $40 \mathrm{~nm} \mathrm{Au} / \mathrm{Pt}$ samples, respectively. These observations suggest that the thermal transport channel in samples with the Ti layer that increases the e-p coupling mechanism is not present in the samples with the Pt layer. Choi et. al [117] recently measured the lower limit to $h_{\mathrm{ee}}>5$ $\mathrm{GW} \mathrm{m}{ }^{-2} \mathrm{~K}^{-1}$ across a Au/Pt interface. Therefore, the Au/Pt interface provides negligible resistance (compared to the resistance due to the slow e-p equilibration in the Au layer).

As previously mentioned, electron-metal/phonon non-metal energy transfer in these $\mathrm{Au} / \mathrm{Pt}$ samples can be ruled out as the metal films are not strongly bonded with the nonmetal interfaces. Therefore, the only resistances to electronic thermal transport in these samples are due to the volumetric e-p coupling in Au and Pt layers. With the knowledge of effective e-p conductance in the Au layer and the total conductance, $G_{\text {eff }} d$, measured by the TTM, the e-p coupling in the Pt layer can be predicted as these conductances are added in series. This procedure predicts $G_{\text {eff }} \sim 4-6 \times 10^{17} \mathrm{~W} \mathrm{~m}^{-3} \mathrm{~K}^{-1}$ for Pt which is lower than the previously measured value in Ref. [120]. This discrepancy is largely due to the use of a linear temperature dependence of the electronic heat capacity to electron temperature $\left(C_{\mathrm{e}}=\gamma T_{\mathrm{e}}\right)$ in Ref. [120] for Pt. Whereas, in this work, $C_{\mathrm{e}}\left(T_{\mathrm{e}}\right)$ values calculated from $a b$ initio calculations in Ref. [58] are used as input parameters in the TTM, the values of which deviate as much as two fold for $T_{\mathrm{e}} \sim 1000 \mathrm{~K}$ from $C_{\mathrm{e}}=\gamma T_{\mathrm{e}}$ with $\gamma=748 \mathrm{~J} \mathrm{~m}^{-3} \mathrm{~K}^{-2}$ [58].

With these findings, going back to Fig. 3.2, it can be confidently asserted that the measured values of $G_{\text {eff }}$ in the $\mathrm{Au} / \mathrm{Ti}$ films are due to the conductance $h_{\mathrm{ei}}$, which is ascribed as a combination of both e-p coupling in the interfacial Ti layer and e-p conductance across the $\mathrm{Ti} /$ substrate interface. Although it is difficult to deconvolute these two conductances, to un- 


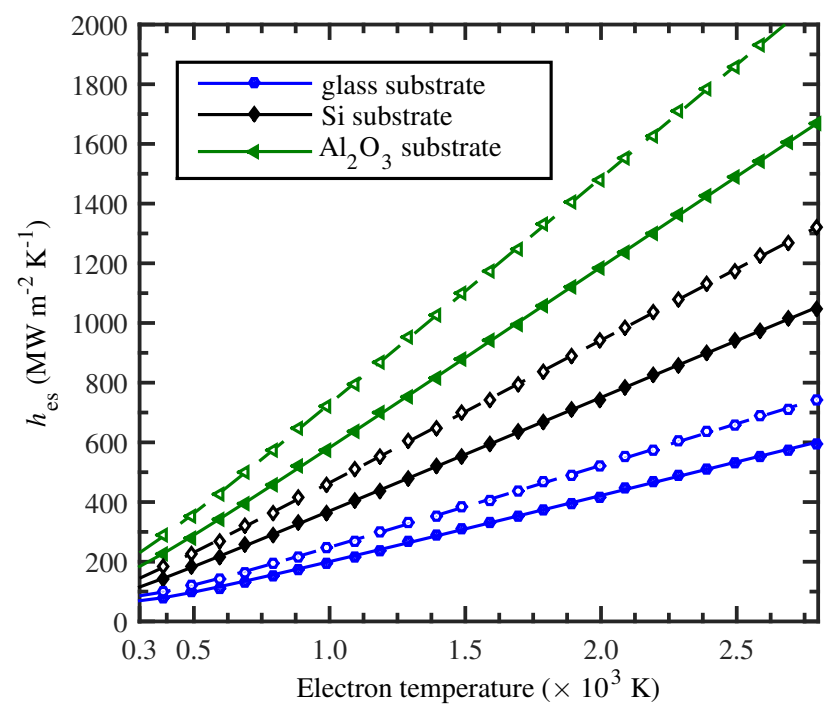

Figure 3.4: $h_{\mathrm{es}}$ as a function of effective electron temperature for three different substrates at $T_{p}=300 \mathrm{~K}$. The closed symbols represent calculations with parameters for Au electrons whereas the hollow symbols represent calculations with parameters for Ti electrons. The values of the predicted $h_{\mathrm{es}}$ are different for different phonon dispersions of the substrate and are in good qualitative agreement with the conductances measured in Fig. 3.2 for the samples with the Ti adhesion layer.

derstand the temperature dependence of the hot metal-electron/non-metal phonon thermal boundary conductance, the model presented in Chapter 2.1.1 is revisited, which predicts "hot" electron-interface thermal boundary conductance. To reiterate, this model presents a coupled thermodynamic and quantum mechanical derivation of e-p scattering at free electron metal/nonmetal substrate interfaces [54]. Figure 3.4] shows the model calculations for electron-metal/phonon-nonmetal interactions for the three substrates studied. Calculations are carried out at a lattice temperature of $300 \mathrm{~K}$ assuming a Debye approximation for the phonon spectra in each substrate. For calculations, the transverse and longitudnal velocities of $3740 \mathrm{~m} \mathrm{~s}^{-1}$ and $5980 \mathrm{~m} \mathrm{~s}^{-1}$ for glass and $6450 \mathrm{~m} \mathrm{~s}^{-1}$ and $10890 \mathrm{~m} \mathrm{~s}^{-1}$ for sapphire, respectively [121, 122]. The details of the calculations for Si are given in Ref. [54]. The closed symbols and solid lines represents calculations carried out for Au electrons scattering with the various substrates, whereas, the open symbol and dotted lines represents calculations for Ti electrons scattering with the interface. As is clear in Fig. 3.4, the model 
predicts linear trends for $h_{\mathrm{es}}$ as a function of electron temperature which is in good qualitative agreement to the trends in the e-p coupling shown in Fig. 3.2 for samples described in Table 3.1. It should also be noted that the model captures the substrate dependent increase in conductances similar to that in Fig. 3.2. Based on the discussions from the previous paragraph and the qualitative agreement between the model and the experimental results, heat flow through $\mathrm{Au} /$ substrate interfaces could be enhanced through the inclusion of a very thin $\mathrm{Ti}$ adhesion layer that allows the electrons to exchange energy with the phonons in the substrate.

This is further supported by the fact that the metal/metal interface as the origin of the linear electron temperature dependence is ruled out in the data, supporting this hypothesis that the temperature dependence observed in the $G_{\text {eff }}$ for the Au/Ti systems in Fig. 3.2 is due to hot electrons in the metal coupling to vibrations in the substrate. Consequently, it has been shown that the presence of a $\mathrm{Ti}$ adhesion layer between $\mathrm{Au}$ and a dielectric substrate can effectively control heat flow within these nanosystems during e-p nonequilibrium. Note that the substrate dependence in the experimental results could also arise due to the thin Ti layer chemically reacting differently with the various substrates, which could in effect change the amount of metallic Ti at the interface. This would lead to a different volumetric coupling between electrons and the Ti lattice for the different dielectric substrates studied in this work. Therefore, the evidence in Fig. 3.2 clearly demonstrates the increase in e-p coupling in $\mathrm{Au} / \mathrm{Ti}$ films compared to Au films, which is attributed to an increased coupling in the interfacial Ti region and/or across the metal/non-metal interface during e-p equilibration.

\subsection{Thermal diffusion processes after electron-phonon equi- libration}

To further understand the heat distribution and diffusion processes in these nanosystems, the TDTR data for samples with the Ti adhesion layer (on various substrates) for a 


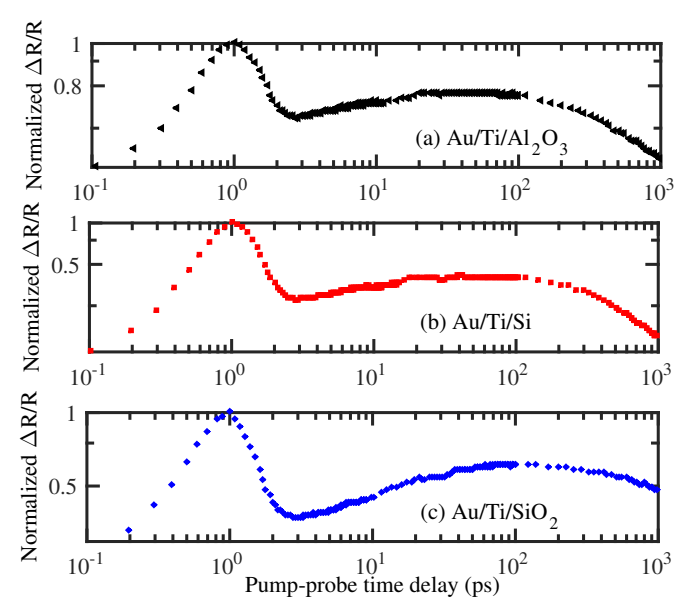

Figure 3.5: TDTR data for the initial $1000 \mathrm{ps}$ after laser pulse absorption for (a) $40 \mathrm{~nm}$ $\mathrm{Au} / \mathrm{Ti} / \mathrm{Al}_{2} \mathrm{O}_{3}$ and (b) $40 \mathrm{~nm} \mathrm{Au} / \mathrm{Ti} / \mathrm{Si}$ and (c) $40 \mathrm{~nm} \mathrm{Au} / \mathrm{Ti} / \mathrm{SiO}_{2}$. The TDTR signal decays rapidly for the first few picoseconds and is related to the electron-phonon coupling process in the thin films. For the 10 to $100 \mathrm{ps}$ time range, samples with the Ti layer show a slow rise in the signal which is attributed to the heating of the Au layer due to heat flow from the Ti layer underneath. This is in contrast to the decreasing TDTR signal (and oscillating picosecond acoustic signal) shown in Fig. 2.6 for Au deposited directly on Si without a Ti adhesion layer.

pump-probe delay time of up to $1 \mathrm{~ns}$ is plotted in Fig. 3.5. A qualitative analysis of the magnitude of the TDTR signal over the entire time scale from pump absorption to $1000 \mathrm{ps}$ provides direct insight into the various thermal processes occurring within these temporal regimes in the metal films since the change in the reflectivity measured in the experiments is related to the sample surface temperature. For example, the first few picoseconds after laser absorption is marked by a fast transient decay of the TDTR signal, which is related to electronic thermalization as described previously. The fast e-p coupling in the Ti layer leads to the increase in the thermal energy of the Ti lattice at a time scale of a few picoseconds after laser pulse absorption (Fig. 3.1). After the electronic system has equilibrated with the lattice vibrations, at time scales from 10 to $50 \mathrm{ps}$, the thermal energy has not yet diffused across the metal bi-layer/substrate interface via phonon-phonon mediated transport since energy diffusion due to this process occurs with at least an order of magnitude larger time constant $\left(\tau_{\text {interface }}=d C / h_{\mathrm{K}}\right.$, Ref. [19]). Due to the relatively larger time constant for the thermal energy to flow across the metal bi-layer/substrate interface through lattice vi- 
brations, the thermal energy from the Ti lattice is transferred to the relatively colder $\mathrm{Au}$ lattice. Consequently, the increase in the temperature of the Au layer leads to an increase in the thermoreflectance signal at these time scales as shown in Fig. 3.5 for $40 \mathrm{~nm} \mathrm{Au/Ti}$ systems, similar to the processes discussed in Ref. [117]. In comparison to Fig. 2.6, where the TDTR data at time scales of 10-100 ps is representative of longitudinal displacement of the weakly adhered Au film on the silicon substrate driven by strain induced from the sudden heating event created by the laser pulses [123], the rise in the thermoreflectivity signal for the samples with the Ti layer suggests that thermal transport in these systems is very different compared to homogeneous thin films. More specifically, thermal diffusion in these $\mathrm{Au} / \mathrm{Ti}$ samples originates in the interfacial layer between the $\mathrm{Au}$ and the substrate due to thermalization of the ballistic electrons that scatter in the Ti layer and at the Ti/non-metal interface. It should be noted that the thermoreflectance signal for the $20 \mathrm{~nm} \mathrm{Au} / \mathrm{Pt}$ sample (as shown in Fig. 3.3a) does not show this increase in the TDTR signal after e-p equilibration even though Pt has a higher e-p coupling factor than Au. This is due to the fact that the effective resistances in each layer due to e-p scattering in the thin film limit, given as $1 / G d$, are comparable, and as a result, the $20 \mathrm{~nm} \mathrm{Au} / \mathrm{Pt}$ system is heated homogeneously. However, for the $40 \mathrm{~nm} \mathrm{Au} / \mathrm{Pt}$ sample, the e-p resistance provided by the Au layer is lower than that in the Pt layer, therefore, there is a slight increase in the thermoreflectance signal from 10-100 ps as shown in Fig. 3.3b.

These results can help shed more light on how the increase in e-p coupling due to the Ti adhesion layer affects the total conductance $\left(h_{\mathrm{K}}\right)$ across the various $\mathrm{Au} / \mathrm{Ti} / \mathrm{substrate}$ interfaces at longer time scales when the electronic and vibrational states in the metal are in near thermal equilibrium (pump-probe time delays from hundreds of picoseconds to several nanoseconds). The TBCs for the samples deposited on $\mathrm{Si}$ and $\mathrm{Al}_{2} \mathrm{O}_{3}$ substrates (described in Table 3.1) are measured by analyzing the TDTR data over a time scale of 100 to 1000 ps. For the $\mathrm{Au} / \mathrm{Al}_{2} \mathrm{O}_{3}$ and $\mathrm{Au} / \mathrm{Ti} / \mathrm{Al}_{2} \mathrm{O}_{3}$ samples described in Table 3.1, we measure $h_{\mathrm{K}}$ values of $35 \pm 4 \mathrm{MW} \mathrm{m}^{-2} \mathrm{~K}^{-1}$ and $215 \pm 15 \mathrm{MW} \mathrm{m}^{-2} \mathrm{~K}^{-1}$, respectively and for the $\mathrm{Au} / \mathrm{Si}$ and $\mathrm{Au} / \mathrm{Ti} / \mathrm{Si}$ samples, we measure $h_{\mathrm{K}}$ values of $88 \pm 8 \mathrm{MW} \mathrm{m}^{-2} \mathrm{~K}^{-1}$ and 
$178 \pm 15 \mathrm{MW} \mathrm{m}^{-2} \mathrm{~K}^{-1}$, respectively. Although $h_{\mathrm{K}}$ measured on this nanosecond time scale demonstrates a large increase with the inclusion of the Ti layer, similar to $G_{\text {eff }}$ measured on the picosecond time scale, it can not be concluded that these two conductance channels are related. For example, if electron-interface scattering from the nonequilibrium regime were influencing the measured $h_{\mathrm{K}}$, it would be expected that $h_{\mathrm{K}}$ would vary with $G_{\text {eff }}$, which has both thickness and fluence dependence based on the energy of the hot, ballistically traveling electrons that scatter in the interfacial region. Along these lines, the measured TBCs for the $40 \mathrm{~nm} \mathrm{Au} / \mathrm{Ti} /$ sapphire and $40 \mathrm{~nm} \mathrm{Au} / \mathrm{Ti} / \mathrm{Si}$ were within the uncertainties reported for the $20 \mathrm{~nm} \mathrm{Au} / \mathrm{Ti}$ sample, indicating that no thickness dependnece is observed in $h_{\mathrm{K}}$ after the electrons and phonons have equilibrated. Also, the TBC for the $40 \mathrm{~nm}$ $\mathrm{Au} / \mathrm{Ti}$ samples showed no fluence dependence suggesting that the electron energy density does not influence the TBCs for these samples at longer pump-probe delay times $(\sim 1$ ns). These observations support the results from previous works that have shown that TBC across metal/nonmetal interfaces is dominated by phonon-phonon mediated processes at the nanosecond time regime [17, 18].

The results presented in this chapter suggest that only at highly nonequilibrium conditions between the electrons and phonons $\left(T_{\mathrm{e}} \gg T_{\mathrm{p}}\right)$ does $h_{\mathrm{ei}}$ contribute to thermal transport for our $\mathrm{Au} / \mathrm{Ti}$ samples. At time scales when the electrons have completely thermalized with the lattice $\left(T_{\mathrm{e}} \sim T_{\mathrm{p}}\right), h_{\mathrm{ei}}$ does not influence thermal transport. The improved phononphonon conduction at the interface due to the $\mathrm{Ti}$ adhesion layer can be partially attributed to improved bonding between the film and the substrate as explained in Chapter 2.2.1 where it is shown that the increase in the thermal energy carrying phonons to higher frequencies at the interfacial region in the side with the lower cutoff frequency results in a higher thermal boundary conductance due to an increase in interfacial bond strength. In this context, several previous works have also demonstrated the increase in phonon-phonon conductance resulting from a stronger interfacial bond between two materials [11, 13, 17, 124]. In fact, a recent work from our group has shown that this enhancement in phonon-phonon coupling at $80 \mathrm{~nm} \mathrm{Au} / \mathrm{Ti} / \mathrm{Si}$ interfaces compared to $\mathrm{Au} / \mathrm{Si}$ interfaces, which is analyzed in terms of 
an increase in adhesion [17].

In addition to the strengthening of the interfacial bond leading to an increase in the overall $h_{\mathrm{K}}$, the phonon spectrum in the thin Ti layer could (to a certain extent) play a role in the enhancement of TBC into the substrate. It is well known that a better overlap of low frequency phonon modes can lead to better phonon transmission and an increase in $h_{\mathrm{K}}$ [125], which the addition of an interfacial Ti layer that has a greater spectral overlap with the $\mathrm{Si}$ and $\mathrm{Al}_{2} \mathrm{O}_{3}$ substrates compared to $\mathrm{Au}$, would produce.

\subsection{Summary}

In summary, the role of titanium adhesion layers at the interface of a gold film and non-metal substrate on the rate of electron-phonon energy equilibration has been investigated. The addition of a $\sim 3 \mathrm{~nm}$ Ti adhesion layer is shown to increase the magnitude of electron-phonon coupling by as much as a factor of five. By studying systems with different substrates having varying thermal effusivities and phonon dispersions, it is shown that this enhanced conductance can be attributed to ballistic electrons in the Au coupled to phonons in the interfacial region. The coupling can be attributed to either electron-phonon scattering in the thin Ti layer or electron-phonon scattering across the metal/non-metal interface. Moreover, it is found that at elevated electron and lattice temperatures, the electron-phonon coupling increases due to an increase in the electron-electron scattering. By analyzing the full temporal regime of the TDTR data from pump absorption to 1 nanosecond, the ballistic electron transport and electron-phonon coupling can be related to the diffusive thermal conductance after electron-phonon equilibrium. This diffusive transport process occurs in two stages: i) energy deposited in the Ti interfacial region flowing "back" and heating up the $\mathrm{Au}$ film followed by ii) thermal boundary conductance from the Au/Ti into the non-metal. The inclusion of the Ti layer enhances the thermal boundary conductance between the metal films and the dielectric substrates, which we attribute in part to the strengthening of the interfacial bond between the metal film and the substrate and also to 
the phonon spectrum in the Ti layer, consistent with prior works (Refs. [17, 125]). The electron-metal/phonon non-metal energy transfer does not influence the thermal boundary conductance across metal/nonmetal interfaces when the electrons have fully thermalized with lattice vibrations. These results suggest that only during highly nonequilibrium conditions between the electrons and phonons $\left(T_{\mathrm{e}} \gg T_{\mathrm{p}}\right)$ does eletron-phonon scattering at an interface contribute to thermal boundary conductance. 


\section{Chapter 4}

\section{Thermal transport in hybrid}

\section{superlattices}

In the previous chapter, it is conclusively shown that when the electrons have thermalized with the lattice, thermal boundary conductance is solely driven by phonon-phonon mediated processes. In the remainder of this dissertation, the phonon-driven thermal transport across hybrid and amorphous-based interfaces is studied. The focus of this chapter will be the study of $\mathrm{ZnO}$ - and $\mathrm{TiO}_{2}$-based hybrid superlattices via time domain thermoreflectance.

Nanomaterial interfaces and concomitant thermal resistances are generally considered as atomic-scale planes that scatter the fundamental energy carriers. Given that the nanoscale structural and chemical properties of solid interfaces can strongly influence this thermal boundary conductance, the ballistic and diffusive nature of phonon transport along with the corresponding phonon wavelengths can affect how energy is scattered and transmitted across an interfacial region between two materials. In hybrid composites composed of atomic layer building blocks of inorganic and organic constituents, the varying interaction between the phononic spectrum in the inorganic crystals and vibronic modes in the molecular films can provide a new avenue to manipulate the energy exchange between the fundamental vibrational energy carriers across interfaces. This chapter systematically studies 
the heat transfer mechanisms in hybrid superlattices of atomic- and molecular-layer-grown zinc oxide or titanium dioxide and hydroquinone with varying thicknesses of the inorganic and organic layers in the superlattices. A large portion of this chapter appeared in two publications in Physical Review B: "Heat-transport mechanisms in molecular building blocks of inorganic/organic hybrid superlattices" [126] and "Reduction in thermal conductivity and tunable heat capacity of inorganic/organic hybrid superlattices" [127].

\subsection{Background}

The introduction of material interfaces in solid nanocomposites has provided the opportunity for user-defined thermal transport in nanosystems through manipulation of the fundamental carriers of heat. The inclusion of these interfaces gives rise to both phononboundary scattering, effectively reducing the thermal conductivity of the solid due to classical size effects [128], and/or partial transmission of thermal energy across the interface driving the thermal boundary conductance [21,22]. To atomistically manipulate the phonon thermal conductivity of a nanosystem with a high density of material interfaces, an understanding of the interplay and relationship of phonon-boundary scattering and thermal boundary conductance across the interfaces must be understood; it is important to note that the reduction in the thermal conductivity of a material due to phonon boundary scattering may not be entirely correlated to the intrinsic thermal boundary conductance between two solids, and is influenced by ballistic transport and phonon mean free paths incident upon the interface, as has been shown both computationally [129] and experimentally [4]. Given that the structural and chemical properties of solid interfaces can strongly influence the thermal boundary conductance [13], the ballistic or diffusive nature of phonon transport, along with the corresponding phonon wavelengths [125], can affect how energy is scattered and/or transmitted across an interfacial region between two materials. This ballistic to diffusive crossover of phonon transport and energy transmission across an atomically thin interface is poorly understood. 
The consideration of these ballistic and diffusive interfacial phonon energy transport processes has major implications for the development of novel nanomaterials for applications such as thermoelectric energy conversion [130-132], where careful placement of interfaces has proven to be useful in efficiently lowering the phononic conductivity while still maintaining electronic conductivity. As an example of a novel class of nanocomposites of recent interest, hybrid organic-inorganic nanomaterials grown by a combined alternation of atomic layer deposition (ALD) and molecular layer deposition (MLD) have exhibited enhanced electrical, optical, magnetic and mechanical functionalities compared to conventional organic or inorganic materials [42, 133-135]. For example, using this ALD/MLD technique, inorganic/organic superlattices (SLs) have shown promise as potential thermoelectric materials [43, 136]. However, paramount in advancing ALD/MLD hybrid structures for use in thermoelectric, or other applications, is an understanding of the phonon transport and scattering processes in these materials; referring to ALD/MLD SLs, this requires understanding phonon scattering at the ALD/MLD boundary, and its correlation with phonon transmission and resulting boundary conductance across the molecular interface. There have been limited previous works focusing on measurements of thermal conductivity of ALD/MLD grown materials [44, 127, 136, 137]. Given the high density of molecular interfaces in these composite systems, advances in the thermophysics of these materials rely on understanding the thermal conductance across the molecular interfaces.

Given the recent interest in thermal transport in organic-based nanocomposites [9, 10 , 138-140] and heat transport across molecular interfaces [11, 124, 141-145], systematically studying the thermal conductivity and energy storage potential (quantified by the heat capacity, $C$ ) of a series of ALD/MLD-grown hybrid SLs also provides an ideal platform to advance our understanding of phonon scattering at, and heat transfer across, thin molecular interfaces. These high quality hybrid nanosystems also provide ideal materials to understand the heat transfer mechanisms in organic/inorganic SLs, and the interplay between phonon-boundary scattering and thermal boundary conductance across interfaces of identical materials separated by a well defined molecular layer. 


\subsection{Experimental details}

\subsubsection{Sample fabrication and characterization}

Hybrid SLs of $\left[\left(\mathrm{TiO}_{2}\right)_{m}\left(\mathrm{Ti}-\mathrm{O}-\mathrm{C}_{6} \mathrm{H}_{4}-\mathrm{O}-\right)_{k=1}\right]_{n}$ with $m=400,40$ and 4 were fabricated on $\mathrm{MgO}$ substrates at $210^{\circ} \mathrm{C}$ via the $\mathrm{ALD} / \mathrm{MLD}$ technique [146] from $\mathrm{TiCl}_{4}, \mathrm{H}_{2} \mathrm{O}$ and hydroquinone (HQ) precursors (Picosun R-100 ALD reactor); a detailed description of the fabrication process for these samples is given in Refs. [147] and [137]. Additionally, $\left[(\mathrm{ZnO})_{m}\left(\mathrm{Zn}-\mathrm{O}-\mathrm{C}_{6} \mathrm{H}_{4}-\mathrm{O}-\right)_{k=1}\right]_{n}$ with $m=99,49,29,9$ and 4 were also fabricated on $\mathrm{Al}_{2} \mathrm{O}_{3}$ substrates. Diethyl zinc and water were used as precursors for the $\mathrm{ZnO}$ layers. Other pertinent details of the fabrication process for the ZnO-based SLs are reported in Ref. [136]. Along with the SL films, control samples of purely ALD grown inorganic $\mathrm{TiO}_{2}$ and $\mathrm{ZnO}$ films with similar thicknesses as the SL samples were also fabricated. All ALD/MLD fabrication and characterization were conducted by our colleagues at Aalto University (Professor Maarit Karppinen).
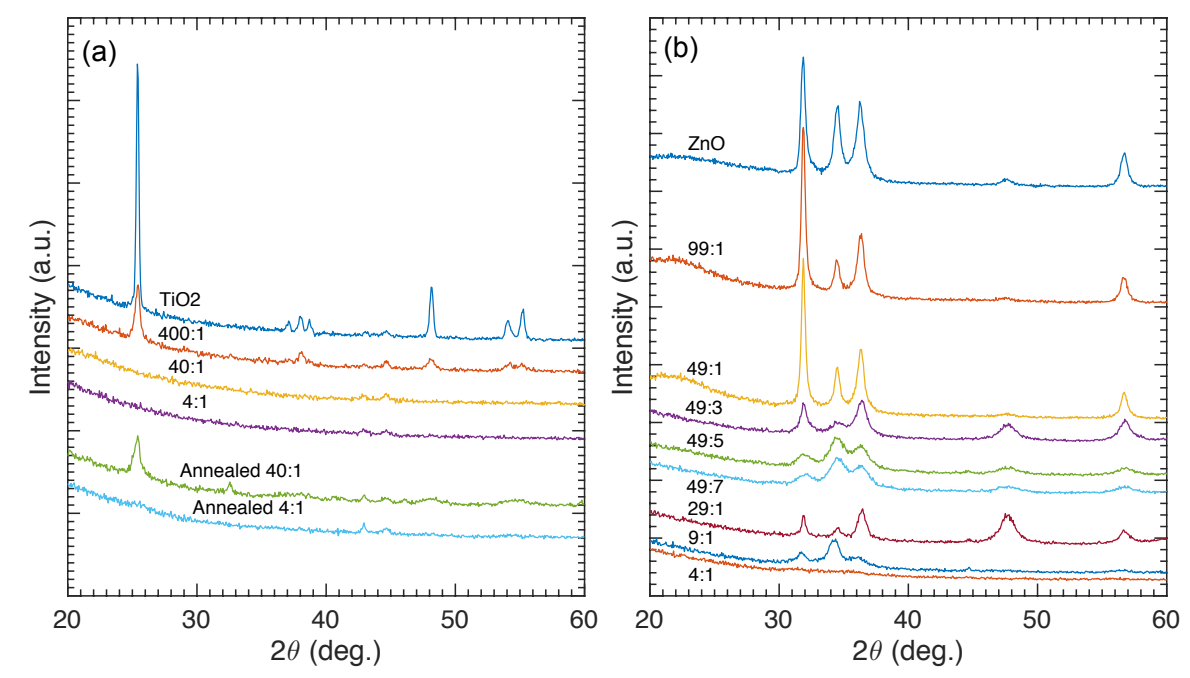

Figure 4.1: Characteristic grazing incidence X-ray diffraction (GIXRD) patterns for the control samples and hybrid SLs with varying ALD/MLD ratios for (a) $\mathrm{TiO}_{2}$-based films and (b) $\mathrm{ZnO}_{2}$-based films.

Figure 4.1 shows the characteristic grazing incidence X-ray diffraction (GIXRD) pat- 
terns for the films tested in this study. The fact that the intensities of the peaks for the as deposited $\mathrm{TiO}_{2}$-based SLs are reduced compared to the purely ALD grown $\mathrm{TiO}_{2}$ with anatase phase, suggests that the crystallinity in those samples is hindered due to the inclusion of the HQ layers. In contrast, for the ZnO-based SLs, the peaks in the XRD patterns for the hybrid SLs fit to the typical hexagonal wurtzite structure of $\mathrm{ZnO}$. There are no shifts in the position of the peaks for the hybrid SLs with ALD:MLD cycle ratios of 99:1, 49:1, 29:1 and 9:1, suggesting that the introduction of the organic monolayers does not affect the crystallinity of the $\mathrm{ZnO}$ phase. The $m=4$ samples for both $\mathrm{ZnO}$ and $\mathrm{TiO}_{2}$-based SLs are amorphous in nature.

In order to enhance the crystallinity of the $\mathrm{TiO}_{2}$-based SLs, the samples were heattreated at $600^{\circ} \mathrm{C}$ for 6 hours (Nabertherm GMbH RS 80/500/11). For the $k: m=1: 40 \mathrm{TiO}_{2}$ based SL (with inorganic period thickness of $\sim 2 \mathrm{~nm}$ ), the high annealing treatment leads to enhanced crystallinity, whereas, for the $k: m=1: 4 \mathrm{TiO}_{2}$-based SL, GIXRD pattern does not show any change compared to the 1:4 as-deposited SL. Furthermore, the annealing process converts the molecular HQ components to a 2D graphitic layer. This conversion was confirmed via Raman spectroscopy and further details on the characterization of the carbon content in the fine internal interfaces for these hybrid SLs can be found in our previous report [137]. Note, this annealing treatment does not affect the periodic layering of the SLs as demonstrated by the X-ray reflectivity (XRR; PANanalytical X'Pert Pro MPD diffractometer) patterns with interference maxima due to SL reflections as shown in Fig. 1d of Ref. [137] for the $\mathrm{TiO}_{2}$-based SLs with $k: m=1: 200$ and 1:400. However, as the layer spacing is inversely proportional to the XRR angle $\theta$, and because the reflected intensity decays exponentially with increasing $\theta$, we do not observe the SL reflections for the $\mathrm{TiO}_{2}$ based hybrid films with very small layer spacing $(k: m=1: 4$ and 1:40) as shown in Fig. 4.2a. In theory, these hybrid films with very small layer spacing should show SL reflections at high $\theta$ angle. Therefore, the measured $\theta$ range for these samples is extended and from the observed peaks in the XRR intensity, it is confirmed that the SLs with $k: m=1: 4$ and 1:40 are in fact layered structures. 

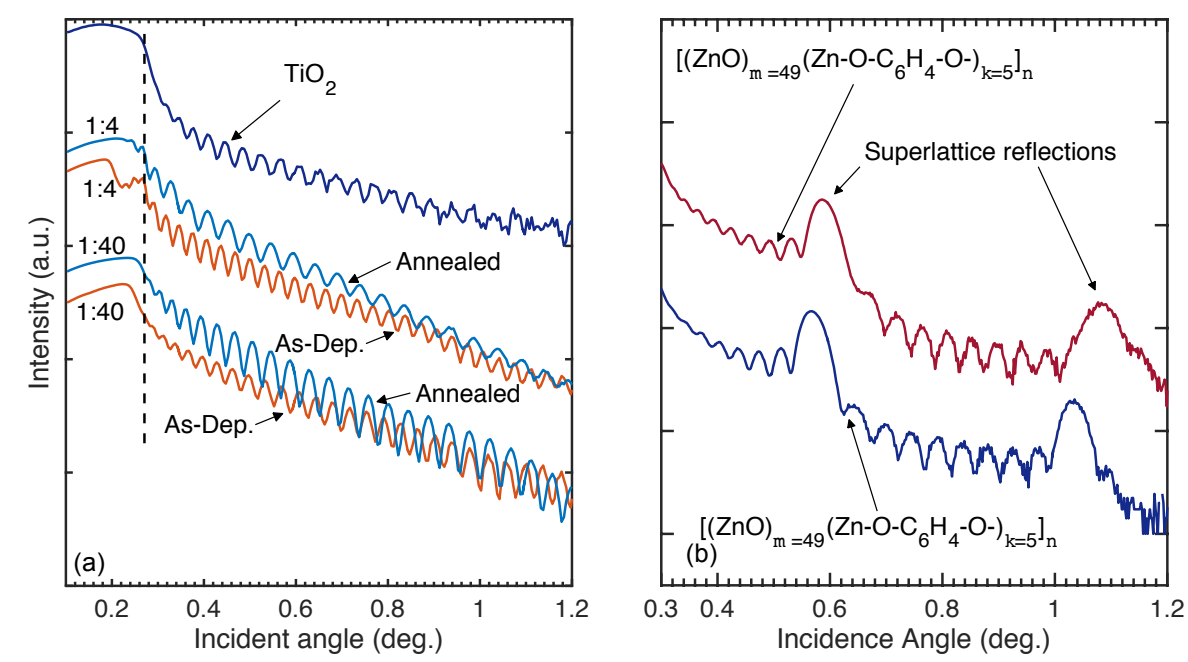

Figure 4.2: Characteristic XRR patterns for the (a) $\mathrm{TiO}_{2}$ - and (b) $\mathrm{ZnO}$ - based films.

The small differences in the XRR patterns seen for the as-deposited and annealed samples reveal a reduction in the film thickness and consequent increase in the film densities. Fig. $4.2 \mathrm{a}$ also highlights the reductions in the film thickness after annealing that underline the shifts in the critical angle $\left(\theta_{c}\right)$ values. The density reduction is expected to mainly stem from the contraction of the organic layers. The densities are estimated from the critical angle values of the XRR patterns [148]. First, the mean electron density values were estimated from $\rho_{e}=\left(\theta_{c}^{2} \pi\right) /\left(\lambda^{2} r_{e}\right)$, where $\lambda$ is the X-ray wavelength and $r_{e}$ is the classical electron radius. Then, assuming elemental compositions to follow the stoichiometry of $\left[\left(\mathrm{TiO}_{2}\right)_{m}\left(\mathrm{Ti}-\mathrm{O}-\mathrm{C}_{6} \mathrm{H}_{4}-\mathrm{O}-\right)_{k}\right]_{n}$ or $\left[(\mathrm{ZnO})_{m}\left(\mathrm{Zn}-\mathrm{O}-\mathrm{C}_{6} \mathrm{H}_{4}-\mathrm{O}-\right)_{k}\right]_{n}$, the mean mass densities were obtained from $\rho_{m}=\left(\rho_{e} A\right) /\left(N_{A} Z\right)$, where $A$ is the average molar mass, $N_{A}$ is the Avogadro constant and $Z$ the average atomic number. The estimated densities along with the measured thicknesses for the thin films are shown in Table 4.1. For the $\mathrm{ZnO}$ based SLs, characteristic XRR patterns for $m=49$ and $k=5$ and 7 are shown in Fig. 4.2p. The film thickness dictates the small fringes corresponding to the interference minima and maxima of the reflected beam film-air and film-substrate interfaces, respectively [147]. The XRR also includes interference maxima with higher intensities that represent constructive interference from the periodic introduction of the organic layers. 
Table 4.1: Thicknesses and densities of the thin films measured via XRR.

\begin{tabular}{lcc}
\hline \hline Sample & Thickness $(\mathrm{nm})$ & Density $\left(\mathrm{g} \mathrm{cm}^{-3}\right)$ \\
\hline $\mathrm{ALD}_{-}$grown $\mathrm{TiO}_{2}$ & & \\
{$\left[\left(\mathrm{TiO}_{2}\right)_{m=40}\left(\mathrm{Ti}-\mathrm{O}-\mathrm{C}_{6} \mathrm{H}_{4}-\mathrm{O}-\right)_{k=1}\right]_{n}$ (as-deposited) } & 110.8 & 3.65 \\
{$\left[\left(\mathrm{TiO}_{2}\right)_{m=40}(\mathrm{C})_{k=1}\right]_{n}($ annealed$)$} & 95.1 & 2.87 \\
{$\left[\left(\mathrm{TiO}_{2}\right)_{m=4}\left(\mathrm{Ti}-\mathrm{O}-\mathrm{C}_{6} \mathrm{H}_{4}-\mathrm{O}-\right)_{k=1}\right]_{n}$ (as-deposited) } & 123.7 & 3.37 \\
{$\left[\left(\mathrm{TiO}_{2}\right)_{m=4}(\mathrm{C})_{k=1}\right]_{n}($ annealed) } & 85.3 & 1.98 \\
$\mathrm{ALD}-$ grown $\mathrm{ZnO}$ & 154.0 & 2.83 \\
{$\left[(\mathrm{ZnO})_{m=99}\left(\mathrm{Zn}-\mathrm{O}-\mathrm{C}_{6} \mathrm{H}_{4}-\mathrm{O}-\right)_{k=1}\right]_{n}$} & 91.1 & - \\
{$\left[(\mathrm{ZnO})_{m=49}\left(\mathrm{Zn}-\mathrm{O}-\mathrm{C}_{6} \mathrm{H}_{4}-\mathrm{O}-\right)_{k=1}\right]_{n}$} & 93.3 & - \\
{$\left[(\mathrm{ZnO})_{m=49}\left(\mathrm{Zn}-\mathrm{O}-\mathrm{C}_{6} \mathrm{H}_{4}-\mathrm{O}-\right)_{k=3}\right]_{n}$} & 81.2 & - \\
{$\left[(\mathrm{ZnO})_{m=49}\left(\mathrm{Zn}-\mathrm{O}-\mathrm{C}_{6} \mathrm{H}_{4}-\mathrm{O}-\right)_{k=5}\right]_{n}$} & 93.1 & - \\
{$\left[(\mathrm{ZnO})_{m=49}\left(\mathrm{Zn}-\mathrm{O}-\mathrm{C}_{6} \mathrm{H}_{4}-\mathrm{O}-\right)_{k=7}\right]_{n}$} & 90.1 & - \\
{$\left[(\mathrm{ZnO})_{m=29}\left(\mathrm{Zn}-\mathrm{O}-\mathrm{C}_{6} \mathrm{H}_{4}-\mathrm{O}-\right)_{k=1}\right]_{n}$} & 97.2 & - \\
{$\left[(\mathrm{ZnO})_{m=9}\left(\mathrm{Zn}-\mathrm{O}-\mathrm{C}_{6} \mathrm{H}_{4}-\mathrm{O}-\right)_{k=1}\right]_{n}$} & 93.8 & 3.3 \\
{$\left[(\mathrm{ZnO})_{m=4}\left(\mathrm{Zn}-\mathrm{O}-\mathrm{C}_{6} \mathrm{H}_{4}-\mathrm{O}-\right)_{k=1}\right]_{n}$} & 82.7 & 2.4 \\
\hline \hline
\end{tabular}

\subsubsection{TDTR measurements and analyses}

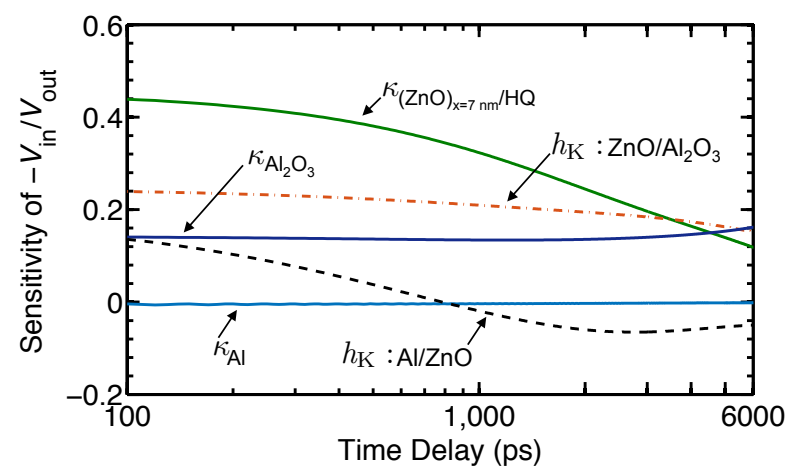

Figure 4.3: Sensitivities of ratio $\left(-V_{\text {in }} / V_{\text {out }}\right)$ to the thermophysical properties of the $(\mathrm{ZnO})_{\mathrm{x}=7 \mathrm{~nm}} / \mathrm{HQ}$ sample as a function of pump-probe time delay at $8.8 \mathrm{MHz}$ pump modulation frequency.

For simplicity, the SL samples will be identified as $(\mathrm{ZnO})_{\mathrm{x}} / \mathrm{HQ}$ (where $\mathrm{x}$ is the period thickness of the SL) for the remainder of the chapter. For the $\mathrm{ZnO}$-based samples, the measured thermal boundary conductances from the control sample (as detailed in Chapter 2.3.1) are used as input parameters for thermal conductivity analyses of the superlattice samples $\left((\mathrm{ZnO})_{\mathrm{X}} / \mathrm{HQ}\right)$. The ratio of the in-phase and out-of-phase signals $\left(-V_{\text {in }} / V_{\text {out }}\right)$ is fit 


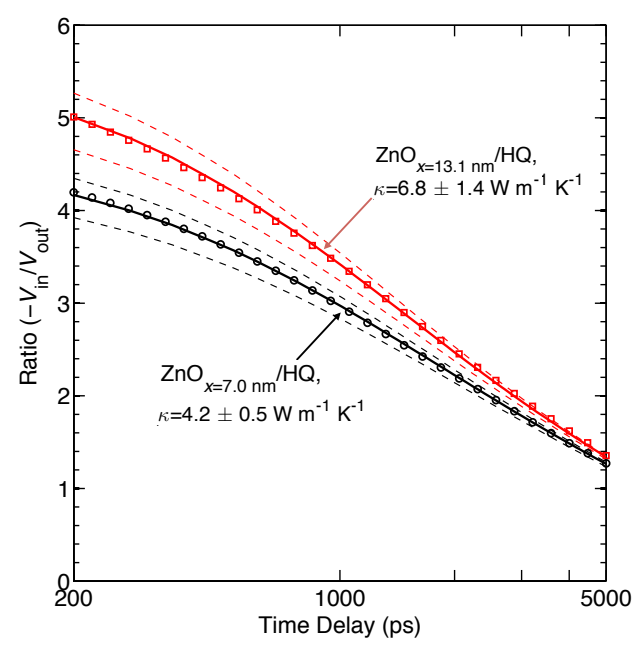

Figure 4.4: Sample data and best-fit curves for the $(\mathrm{ZnO})_{\mathrm{x}=7.0 \mathrm{~nm}} / \mathrm{HQ}$ (red squares) and $(\mathrm{ZnO})_{\mathrm{x}=7.0 \mathrm{~nm}} / \mathrm{HQ}$ (black circles) along with uncertainties (dotted lines) at room temperature.

to the three-layer thermal model to determine the thermal conductivity of the superlattice films. For these fits, the thermal boundary conductances determined from the in-phase analyses are used, leaving the only unknown parameter in these measurements as the thermal conductivity of the $(\mathrm{ZnO})_{\mathrm{X}} / \mathrm{HQ}$ films. Figure 4.3 shows the sensitivity of the ratio to the various parameters in the three-layer model for the $(\mathrm{ZnO})_{\mathrm{x}=7 \mathrm{~nm}} / \mathrm{HQ}$ film. An error of $15 \%$ in $h_{\mathrm{K}}$ for the $\mathrm{Al} / \mathrm{ZnO}$ interface propagates to an error of $\sim 1.5 \%$ and $\sim 0.8 \%$ on the measured thermal conductivities of the $(\mathrm{ZnO})_{\mathrm{x}=13.1 \mathrm{~nm}} / \mathrm{HQ}$ and $(\mathrm{ZnO})_{\mathrm{x}=7.0 \mathrm{~nm}} / \mathrm{HQ}$ samples at room temperature, respectively. However, an error of $15 \%$ in $h_{\mathrm{K}}$ for the $\mathrm{ZnO} / \mathrm{Al}_{2} \mathrm{O}_{3}$ interface causes an error of $\sim 13 \%$ and $\sim 7 \%$ in the measured conductivities for $(\mathrm{ZnO})_{\mathrm{x}=13.1 \mathrm{~nm} / \mathrm{HQ}}$ and $(\mathrm{ZnO})_{\mathrm{x}=7.0 \mathrm{~nm}} / \mathrm{HQ}$ samples at room temperature, respectively. This is a major source of uncertainty reported for our measurements. The fits to the TDTR data along with the uncertainties (dashed lines) for the samples $(\mathrm{ZnO})_{\mathrm{x}=7.0 \mathrm{~nm}} / \mathrm{HQ}$ and $(\mathrm{ZnO})_{\mathrm{x}=13.1 \mathrm{~nm}} / \mathrm{HQ}$ at $300 \mathrm{~K}$ are shown in Fig. 4.4.

For the $\mathrm{TiO}_{2}$-based samples, due to the relatively low thermal conductivity of the films, the most sensitive parameters are the heat capacity $\left(C_{\mathrm{TiO}_{2}: \mathrm{HQ}}\right)$ and thermal conductivity $\left(\kappa_{\mathrm{TiO}_{2}: \mathrm{HQ}}\right)$ of the hybrid SLs (as shown in the sensitivity plots at two different frequen- 
cies in Figs. $4.5 \mathrm{a}$ and $4.5 \mathrm{~b}$ ). The fact that the sensitivities are different and dynamic in nature allows us to simultaneously measure $C_{\mathrm{TiO}_{2}: \mathrm{HQ}}$ and $\kappa_{\mathrm{TiO}_{2}: \mathrm{HQ}}$ as discussed in detail below. The front side interface conductance, $h_{\mathrm{K}, \mathrm{Al} / \mathrm{TiO}_{2}}$, and the back side interface conductance, $h_{\mathrm{K}, \mathrm{TiO}_{2} / \mathrm{MgO}}$, are measured from separate measurements on the control sample $\left(\mathrm{Al} / \mathrm{TiO}_{2} / \mathrm{MgO}\right)$ using a similar procedure as discussed in Chapter 2 and described in more detail in the following paragraph. As shown in Fig. $4.5 \mathrm{a}$ and $4.5 \mathrm{~b}$, the measurements are

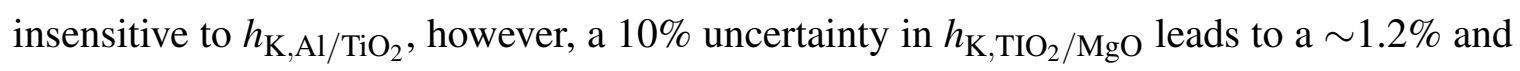
$\sim 2.4 \%$ uncertainty in $\kappa_{\mathrm{TiO}_{2}: \mathrm{HQ}}$ and $C_{\mathrm{TiO}_{2}: \mathrm{HQ}}$ at room temperature for $8.8 \mathrm{MHz}$ freuqency, respectively, which quantifies this insensitivity. The same procedure can be applied for the $\mathrm{ZnO}$-based samples with relatively low thermal conductivities and high density of HQ layers.

The measurements for $h_{\mathrm{K}, \mathrm{Al} / \mathrm{TiO}_{2}}$ and $h_{\mathrm{K}, \mathrm{TiO}_{2} / \mathrm{MgO}}$ are confirmed by analyzing the thermoreflectance data for the control sample using two different approaches. The first approach analyzes $V_{\text {in }}$ as well as $-V_{\text {in }} / V_{\text {out }}$ separately, as we outline in Chapter 2.3.1 for the ALD grown $\mathrm{ZnO}$ control sample. The second method utilizes two frequencies to effectively separate the interfacial conductances. At $3.72 \mathrm{MHz}$ modulation frequency, an
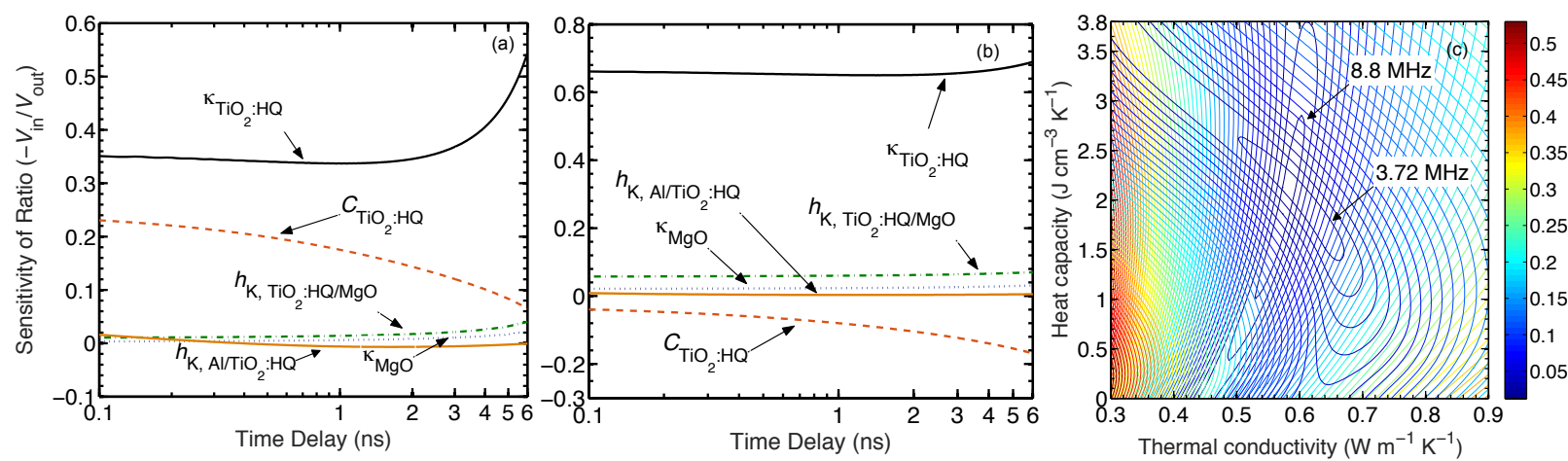

Figure 4.5: Sensitivities of ratio $\left(-V_{\text {in }} / V_{\text {out }}\right)$ to the thermo-physical properties of the AsDep $\left(\mathrm{TiO}_{2}\right)_{\mathrm{x}=0.5 \mathrm{~nm}} / \mathrm{HQ}$ sample as a function of pump-probe time delay at (a) $8.8 \mathrm{MHz}$ pump modulation frequency and (b) $3.72 \mathrm{MHz}$ pump modulation frequency. (c) Sensitivity contour plot showing the interrelationship between thermal conductivity and heat capacity of the As-Dep $\left(\mathrm{TiO}_{2}\right)_{\mathrm{x}=0.5 \mathrm{~nm}} / \mathrm{HQ}$ sample at $3.72 \mathrm{MHz}$ and $8.8 \mathrm{MHz}$ pump modulation frequencies. 
effective thermal conductivity that considers the front side conductance and the thermal conductivity of the hybrid structure as a lumped conductance is estimated. The data is fit to the model with this effective conductivity and the back side conductance as free parameters. For the higher modulation frequency $(8.8 \mathrm{MHz})$, the data is fit with the free parameters as the thermal conductivity of the SL and front side conductance and the back side conductance is used as an input parameter that is determined from fitting the data at the lower modulation frequency. It should be noted that this approach to measuring the front side and back side conductances gives agreeable values to the first method outlined in Chapter 2.3.1. Uncertainties in the measured thermal conductivities and heat capacities of the hybrid SLs are derived from the uncertainties in the input parameters and the sensitivity of the threelayer thermal model to those parameters. Following this procedure, the measured value of $\kappa=5.2 \pm 0.5 \mathrm{~W} \mathrm{~m}^{-1} \mathrm{~K}^{-1}$ for the purely $\mathrm{ALD}$ grown $\mathrm{TiO}_{2}$ film is in good agreement with the literature value of $\sim 5.7 \mathrm{~W} \mathrm{~m}^{-1} \mathrm{~K}^{-1}$ for an anatase thin film [149].

For a given frequency, the best fit to the TDTR signal (for hybrid SLs with high interfacial densities) can be produced with multiple combinations of the heat capacities and thermal conductivities, as shown in the sensitivity contour plots in Fig. $4.5 \mathrm{c}$ for a $k: m=1: 4$, as-deposited $\mathrm{TiO}_{2}$-based SL. The contour plots represent the mean square deviation of the model to the TDTR data with the various combinations of $C$ and $\kappa$ as input parameters in the three-layer model [92]. Note, the best fits to the TDTR data are produced with the residuals $\lesssim 0.02$. As is clear from the sensitivity contour plot, a wide range of values for $C$ can produce the best fit in the least squares sense for a given frequency. Therefore, to accurately determine $C$ and $\kappa, 3.72$ and $8.8 \mathrm{MHz}$ pump modulation frequencies are used, which give different sensitivity contour maps for the thermophysical properties (see Fig. 4.5k). The common set of values for $C$ and $\kappa$ at these two frequencies shown by the overlap of the best fit values represent our measurements for the hybrid SLs. 


\subsection{Thermal conductivity measurements for the $\mathrm{TiO}_{2}$-based SLs}

Figure 4.6 shows the measured thermal conductivities for the as-deposited and annealed $\mathrm{TiO}_{2}$-based samples with $m=40$ as a function of temperature. Two aspects of the results shown in Fig. 4.6 are worth noting. First, the thermal conductivities measured for the asdeposited and annealed SLs show a large reduction as compared to the sputtered $\mathrm{TiO}_{2}$ thin films [150]; at room temperature, $\kappa$ for the as-deposited SL is almost $30 \%$ lower than the measured $\kappa$ for an anatase thin film (Fig. 4.6, sold triangle) [149]. For comparison, the thermal conductivity of bulk, single crystal anatase taken from Ref. [151] is also included. The reason for the decrease in the thermal conductivities is attributed to incoherent boundary scattering at the inorganic/organic interfaces [137]. For comparison, Fig. 4.6 also shows the measured $\kappa$ for an as-deposited amorphous $\mathrm{TiO}_{2}$ film. Even with the enhancement in crystallinity of the annealed sample, boundary scattering at the inorganic/organic/inorganic interfaces results in the low values for thermal conductivities. In Fig. 4.6, the predictions from a minimum thermal conductivity model for a homogeneous amorphous $\mathrm{TiO}_{2}$ are also included. This model assumes that the "mean free paths" of vibrations in the amorphous state are limited to the spacing between the atoms. Therefore, following Ref. [121], the thermal conductivity as a result from random walk between localized oscillators is,

$$
\kappa_{\min }=\left(\frac{\pi}{6}\right)^{1 / 3} k_{\mathrm{B}} n^{2 / 3} \sum_{i} v_{i}\left(\frac{T}{\Theta_{i}}\right)^{2} \int_{0}^{\Theta_{i} / T} \frac{x^{3} e^{x}}{\left(e^{x}-1\right)^{2}} d x
$$

where the sum is taken over the three sound speeds $\left(v_{i}\right), n$ is the atomic density, and $\Theta_{i}=$ $v_{i}\left(\hbar / k_{\mathrm{B}}\right)\left(6 \pi^{2} n\right)^{1 / 3}$ is the cutoff frequency for each polarization expressed in degrees [121]. For the calculations shown in Fig. 4.6 (for a homogeneous $\mathrm{TiO}_{2}$ ), the longitudinal and transverse sound speeds are taken from Ref. [152]. As is clear from Fig. 4.6, the predicted minimum in thermal conductivity for $\mathrm{TiO}_{2}$ agrees very well with our measured values for a homogeneous amorphous $\mathrm{TiO}_{2}$ thin film and the thermal conductivity can be further 
lowered below this minimum limit by the inclusion of periodic monolayers of HQ.

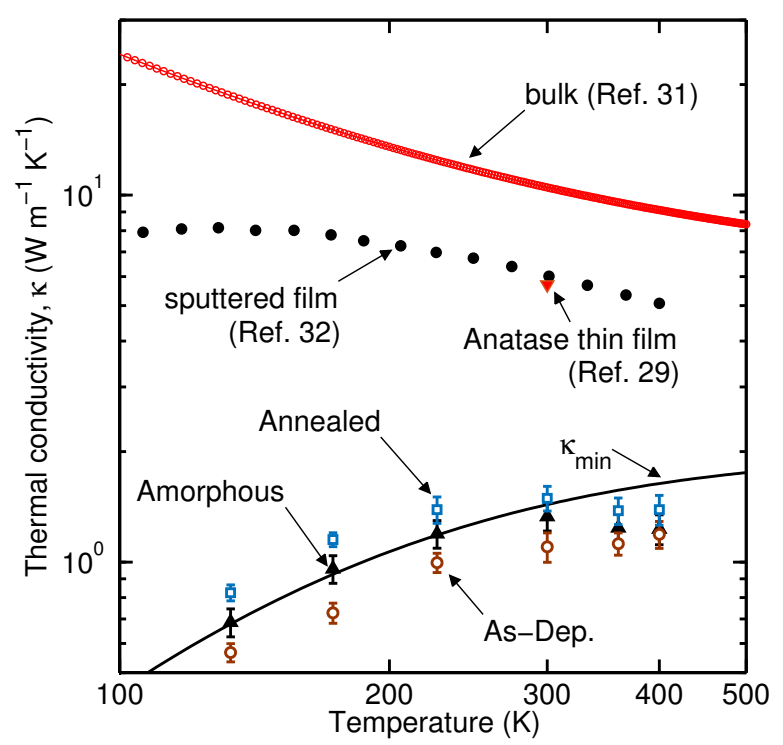

Figure 4.6: Thermal conductivities of as-deposited and annealed $\mathrm{TiO}_{2}$-based $m=40 \mathrm{SLs}$ plotted as a function of temperature. For comparison, the measured thermal conductivities of an ALD grown amorphous $\mathrm{TiO}_{2}$ film along with the thermal conductivities of bulk, single crystal $\mathrm{TiO}_{2}$ (Ref. [151]), a polycrystalline sputtered film with $17 \mathrm{~nm}$ grain size (Ref. [150]) and anatase thin film (Ref. [149]) are also shown. The calculated minimum in thermal conductivity for $\mathrm{TiO}_{2}$ is also shown for comparison.

The second aspect to note in Fig. 4.6 is the increase in the thermal conductivities of the hybrid SLs after annealing at $600^{\circ} \mathrm{C}$ for 6 hours. This can be understood as a consequence of enhanced crystallinity of the inorganic constituents due to the high annealing temperatures. However, the role of the different organic constituents (2D graphitic carbon layers in the annealed samples as compared to the HQ monolayers in the as-deposited samples) might affect the vibrational scattering mechanisms differently at these molecular interfaces. To understand the relative contributions of these two competing effects on thermal conductivity, the results for the annealed and as-deposited samples with $m=4$ are compared. The measured values are $\kappa=0.62 \pm 0.04 \mathrm{~W} \mathrm{~m}^{-1} \mathrm{~K}^{-1}$ and $0.66 \pm 0.04 \mathrm{~W} \mathrm{~m}^{-1}$ $\mathrm{K}^{-1}$ for the as-deposited and annealed samples, respectively. For these samples, even with the high annealing treatments, the inorganic constituents are mostly amorphous in nature, 
as demonstrated by the GIXRD patterns in Fig. 4.1a. Consequently, the values for the measured thermal conductivities are agreeable within uncertainties, suggesting that the role of the organic layers (in these two samples with the same number of organic interfaces) in thermal transport are similar; in other words, the orientation of the HQ layer does not affect scattering at the inorganic/organic boundary and the resulting vibrational thermal conductivity. Contrary to the $m=4$ sample, the crystallinity of the $m=40$ sample is enhanced after annealing as demonstrated by the increase in the 101 peak (see Fig. 4.1a) and, therefore, the increase in $\kappa$ is a consequence of enhanced crystallinity and not due to the intrinsic scattering mechanisms in the organic layers.

\subsection{Thermal conductivity measurements for the $\mathrm{ZnO}$-based}

\section{SLs}

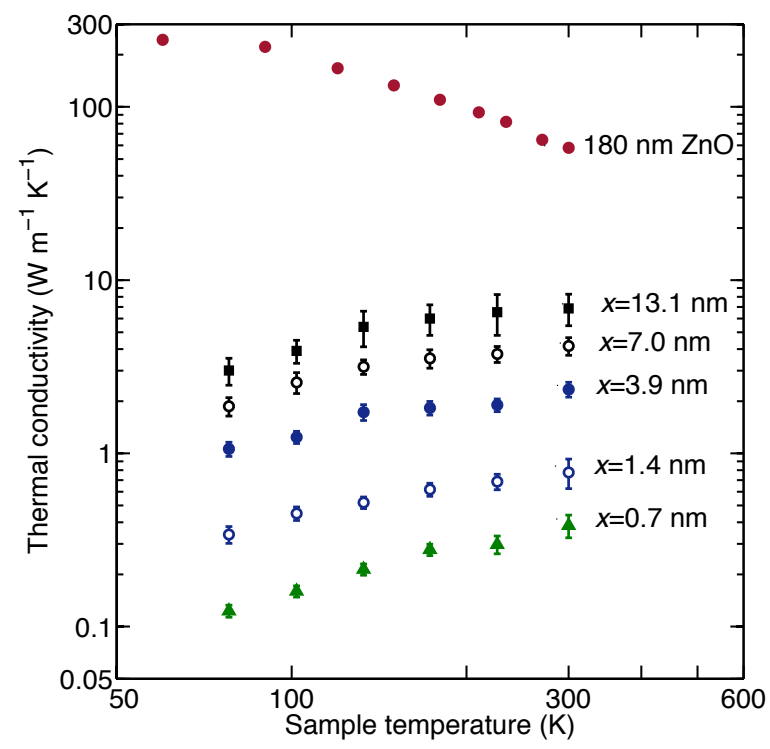

Figure 4.7: Measured thermal conductivities as a function of temperature for $(\mathrm{ZnO})_{\mathrm{X}} / \mathrm{HQ}$ SLs with varying $\mathrm{x}$. The error bars include uncertainties due to repeatability, Al thickness measurement and uncertainties in the parameters used in the thermal model. Also plotted are the thermal conductivities for a $180 \mathrm{~nm}$ ALD-grown homogenous $\mathrm{ZnO}$ film taken from Ref. [91]. 
Figure 4.7 shows the measured thermal conductivities for the $(\mathrm{ZnO})_{\mathrm{X}} / \mathrm{HQ}$ SLs with varying $\mathrm{x}$ at different sample temperatures. The thermal conductivities of these SLs demonstrate more than a ten-fold decrease compared to the results for an ALD-grown homogeneous $\mathrm{ZnO}$ thin film [91] as shown in Fig. 4.7. The inclusion of higher interface densities and the reduction in the inorganic layer thickness results in the reduction of the thermal conductivities of these hybrid SLs, similar to the results for the $\mathrm{TiO}_{2}$-based SLs.

To describe the results in Fig. 4.7, we consider the thermal transport in these hybrid samples being described by a phonon flux in the inorganic material that is limited only by phonon/boundary scattering at the inorganic/organic interface. In other words, it is assumed that the overall thermal conductivities of the SL films are minimally affected by scattering mechanisms in the bulk of the inorganic constituent (such as phonon-defect or phononphonon scattering in the individual layers). Therefore, the thermal transport is limited by the combination of the phonon flux, $q$, in the inorganic layers and the thickness, $\mathrm{x}$, of the layers (i.e., $\mathrm{x}=$ period thickness of the SLs). The phonon flux in the inorganic layer can be approximated by [33],

$$
q=\frac{1}{8 \pi^{2}} \sum_{j} \int_{k_{1}} \hbar \omega k^{2} v_{j} f \mathrm{~d} k
$$

where, $j$ is the polarization, $\omega$ is the phonon frequency, $\hbar$ is Planck's constant, $f$ is the Bose-Einstein distribution and $v$ is the group velocity. Equation 4.2 assumes an isotropic, spherical Brillouin zone to predict the heat flux in the inorganic $\mathrm{ZnO}$ layers. To validate these assumptions, we use the phonon dispersion to calculate the heat capacity, $C$, of $\mathrm{ZnO}$ as,

$$
C=\sum_{j} \int_{0}^{\omega_{c, j}} \hbar \omega D_{j}(\omega) \frac{\partial f}{\partial T} \mathrm{~d} \omega
$$

Figure 4.8 compares the predicted $C$ from our calculations using the fitted phonon dispersion to the experimentally measured $C$ for $\mathrm{ZnO}$ in Ref. [153]. The calculations of Eq. 4.3 for $\mathrm{ZnO}$ agree well with the experimentally measured values, validating our assumptions.

With the flux, $q$, determined from the phonon dispersion, the effective thermal conduc- 


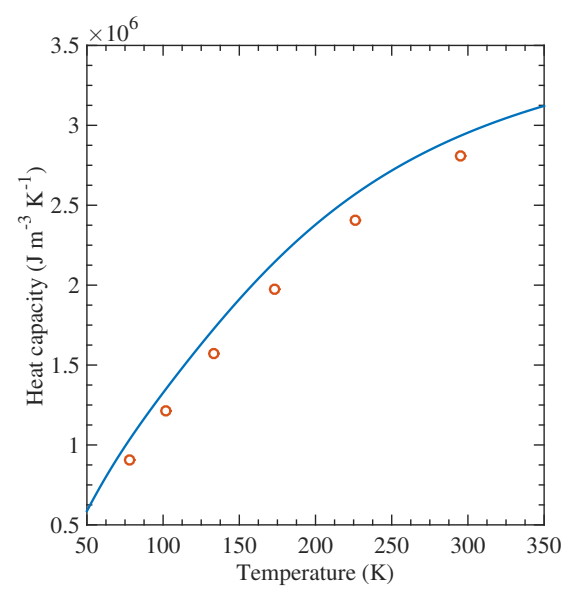

Figure 4.8: Comparison of the experimentally measured heat capacity in Ref. [153] (hollow circles) to the values predicted by Eq. 4.3 (solid line).

tivity of the SLs that is dictated by the period thickness $\mathrm{x}$, is given by,

$$
\kappa_{\text {effective }}=\frac{1}{3} \mathrm{x} \int C_{k} v_{k} \mathrm{~d} k=\frac{\partial q}{\partial T} \mathrm{x}
$$

where $T$ is temperature and $C_{k}$ is the spectral phonon heat capacity. Equation 4.4 assumes that phonon transport in the inorganic layer is ballistic and that the phonons scatter only at boundaries that restore local thermodynamic equilibrium. As such, the discussion and analyses assume that the interfacial organic boundaries are considered to be reflectionless and black, and the phonon flux is assumed to thermalize at these boundaries. Calculations of Eq. 4.4 for $\mathrm{ZnO}$ at two temperatures as a function of $\mathrm{x}$ are shown in Fig. 4.9. For these calculations, all 12 branches of the bulk phonon dispersion relation for $\mathrm{ZnO}$ in the $\Gamma \rightarrow \mathrm{M}$ direction, as calculated in Ref. [154] via ab initio methods are used. The measured thermal conductivities at $78 \mathrm{~K}$ and $300 \mathrm{~K}$ for the SLs show good agreement with the calculations of Eq. 4.4, supporting the assertion that size effects in the inorganic layers of the hybrid SLs limit thermal transport. This analysis assumes that the entire spectrum of phonon mean free paths in the $\mathrm{ZnO}$ layer is limited by scattering at the inorganic/organic/inorganic interface.

The drastic reduction in the thermal conductivity values with decreasing period in the SLs is clearly seen by the inverse relationship of $\kappa$ with $\mathrm{ZnO} / \mathrm{HQ} / \mathrm{ZnO}$ interface density 


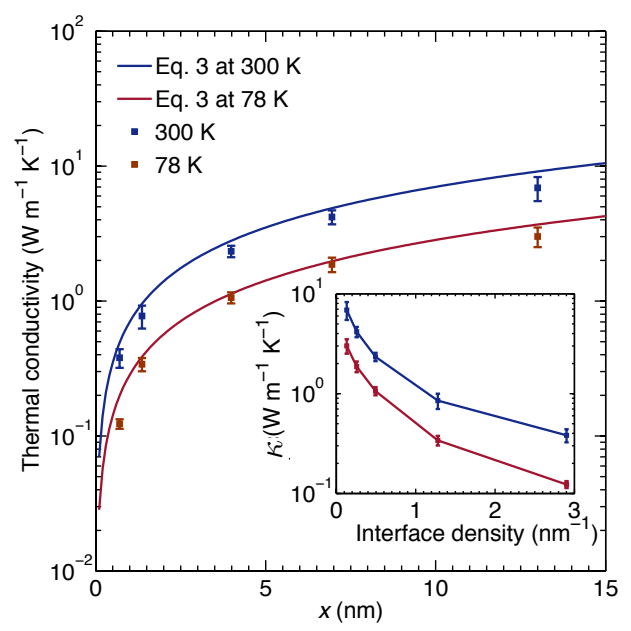

Figure 4.9: Thermal conductivity as a function of the inorganic layer thickness for SLs with single $\mathrm{HQ}$ layers at $78 \mathrm{~K}$ and $300 \mathrm{~K}$. Along with that, the predicted thermal conductivities as a function of SL period thickness calculated from Eq. 4.4 at $78 \mathrm{~K}$ and $300 \mathrm{~K}$ are also shown. The effective thermal conductivity model assuming only phonon-boundary scattering at the HQ-layer interface described in Eq. 4.4 provides reasonable agreement with the measured thermal conductivities for these SLs. (inset) The thermal conductivity for these hybrid SLs is inversely proportional to the $\mathrm{ZnO} / \mathrm{HQ} / \mathrm{ZnO}$ interface density.

as shown in the inset of Fig. 4.9. To scope the generality of these results to hybrid SLs, the measured thermal conductivity of $3.1 \pm 0.2 \mathrm{~W} \mathrm{~m}^{-1} \mathrm{~K}^{-1}$ for a $\left(\mathrm{TiO}_{2}\right)_{\mathrm{x}} / \mathrm{HQ}$ SL with $\mathrm{x}=15.5 \mathrm{~nm}$ at room temperature is compared to the thermal conductivity measurement for a homogeneous $\mathrm{TiO}_{2}$ thin film $\left(5.2 \pm 0.3 \mathrm{~W} \mathrm{~m}^{-1} \mathrm{~K}^{-1}\right)$ [127, 137]. The reduction in thermal conductivity for the $\mathrm{TiO}_{2}$-based SL is in line with the results reported for the $(\mathrm{ZnO})_{\mathrm{x}} / \mathrm{HQ}$ SLs. This reduction in the thermal conductivity due to the periodic monolayers is consistent with the decrease in thermal conductivity with increased interface density in inorganic SLs [155, 156].

As pointed out in purely inorganic SLs, the monotonic decrease in thermal conductivity due to increased interface density (and linearly increasing thermal resistance with increasing interface density) is due to incoherent scattering, where the phonons behave as particles and lose their phase information by scattering at the internal boundaries [155, 156]. Ravichandran et al. [155] have shown that by increasing the interface density (decreasing 
period thicknesses) beyond the incoherent regime, the phonon dispersion in inorganic SLs can be altered by mini-band formation, which effectively preserves the coherent nature of phonon transport in these SLs. An alternative wave nature of phonon transport in inorganic SLs has also been demonstrated by Luckyanova et al. [5], where they varied the total thickness of the inorganic SL films while keeping the SL period thicknesses constant and showed an increase in the thermal conductivity. The results for the hybrid SLs are consistent with the particle nature of phonon transport (or the incoherent scattering regime) as demonstrated by the monotonically decreasing thermal conductivity with increasing interface densities (see inset of Fig. 4.9).

The appreciable agreement between our measured values for a wide range of inorganic layer thicknesses and that predicted by the model in Eq. 4.4 (as shown in Fig. 4.9) suggests that the phonon flux in the inorganic layer is mostly ballistic and the phonon mean free path is limited by the $\mathrm{ZnO}$ layer thicknesses. However, for thicker inorganic layers, where phonon-phonon scattering in the bulk of the inorganic layer creates a temperature gradient along the layer, the validity of Eq.4.4 in describing thermal transport in these SLs is questionable. In fact, this is exemplified by the disagreement between the prediction of

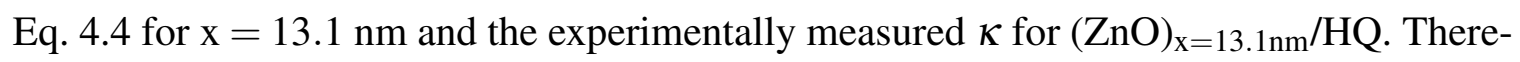
fore, by describing the thermal transport by Eq. 4.4, the thermal conductivities of these hybrid SLs are considered to be driven by a ballistic phonon flux limited by scattering at the inorganic/organic interface, which clearly breaks down as the $\mathrm{ZnO}$ thickness increases. Therefore, to study the validity and range of applicability of this hypothesis, an alternative analysis of the results in Figs. 4.7 and 4.9 is considered by describing the reduction in thermal conductivity to be driven by a thermal boundary conductance across the inorganic/organic/inorganic interface. This approach will give quantitative insight into the role of phonon transmission across the inorganic/organic/inorganic interface on our measured thermal conductivities. Note, as it is not possible to separate the individual resistances due to scattering at the $\mathrm{ZnO} / \mathrm{HQ}$ boundaries and the internal scattering in the $\mathrm{HQ}$ layer, these scattering mechanisms are coupled into a lumped resistance in the discussion and analysis 
presented below.

\subsection{Thermal boundary conductance in organic/inorganic composites}

The AMM and DMM described in Chapter 2.1 could potentially offer complementary, yet alternative insight into the mechanisms driving the large reduction in the measured thermal conductivity of these hybrid SLs, and elucidate the role of the organic monolayers and their intrinsic vibrational properties on phonon transport. The thermal boundary conductance $\left(h_{\mathrm{K}}\right)$ through the organic interface, which is described by the temperature derivative of the phonon flux (as described in Eq.4.2) with the inclusion of a transmission coefficient $\left(\zeta_{1 \rightarrow 2}\right)$ from side 1 to 2 (from inorganic, through the organic monolayer, and emitted into the next inorganic layer). The thermal boundary conductance is defined based on the temperature of the incident and emitted phonons, and therefore it predicts a finite interfacial conductance (as opposed to an infinite conductance or zero thermal boundary resistance) for an imaginary interface comprised of the same material [157]. This conductance occurs when $\zeta_{1 \rightarrow 2}=1$ and all available phonon modes are transmitted from side 1 to 2 of the imaginary interface in the crystal. It should be noted that by this definition, the maximum possible thermal boundary conductance for an imaginary interface is solely limited by the phonon flux that impinges upon the interface. Alternatively, assuming an interface between two materials that causes diffusive scattering, this maximum limit is described by a transmission of $\zeta=0.5$.

To consider the possibility of the thermal boundary conductance across the inorganic/organic/inorganic interface-limiting the thermal transport across the SLs, $h_{\mathrm{K}}$ is modeled across the $\mathrm{ZnO} / \mathrm{HQ} / \mathrm{ZnO}$ interface assuming maximum phonon transmission. This assumption implies that the phonon transmission from the $\mathrm{ZnO}$ across the $\mathrm{HQ}$ is unimpeded by any properties of the HQ; that is, it assumes $\zeta_{1 \rightarrow 2}=1$. For these calculations, it is made sure that the same assumptions for $\mathrm{ZnO}$ density of states and phonon velocities as in Eq. 4.2 

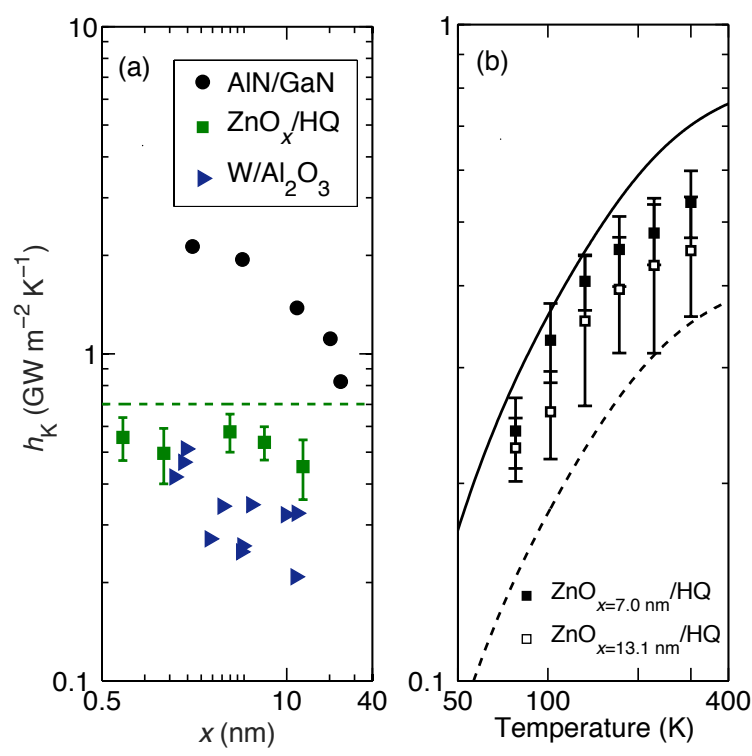

Figure 4.10: (a) The mean thermal boundary conductances of interfaces in $\mathrm{ZnO}_{\mathrm{x}} / \mathrm{HQ}$ SLs derived from thermal conductivity measurements shown in Fig. 4.7. Also plotted are the mean conductances of interfaces in $\mathrm{W} / \mathrm{Al}_{2} \mathrm{O}_{3}$ SLs [158] and AlN/GaN SLs [4] for comparison. (b) The mean thermal boundary conductances derived for $\mathrm{x}=7.0 \mathrm{~nm}$ and $13.1 \mathrm{~nm}$ as a function of temperature for the $\mathrm{ZnO}$ based SLs. The calculations of maximum conductance in $\mathrm{ZnO}$ with phonon transmission coefficient equal to unity are also shown. Also included for comparison is the calculation of the $\mathrm{DMM}$ for a $\mathrm{ZnO} / \mathrm{ZnO}$ interface (i.e., $50 \%$ transmission of the $\mathrm{ZnO}$ phonon flux).

are used. Calculation of this maximum conductance at room temperature for a $\mathrm{ZnO}$ phonon flux is shown in Fig. 4.10a (dashed line). In most real nanosystems, due to both a mismatch of vibrational density of states and imperfections around the interfacial regions, the transmission coefficient is not unity (for a review of thermal boundary conductance dictated by various interfacial conditions, readers are referred to Ref. [13]). For this reason, the measured values of $h_{\mathrm{K}}$ in the literature have never exceeded this maximum thermal boundary conductance for any interface.

From the measured thermal conductivities in the hybrid SLs, we derive the mean thermal boundary conductance across the individual $\mathrm{ZnO} / \mathrm{HQ} / \mathrm{ZnO}$ interfaces with a series resistor model, which assumes that phonons can only scatter at the $\mathrm{ZnO} / \mathrm{HQ} / \mathrm{ZnO}$ interfaces (consistent with the previous analysis where it is assumed that the phonon flux is only scat- 
tered at the $\mathrm{ZnO} / \mathrm{HQ}$ boundaries). The mean conductance across the HQ layers is calculated as $h_{\mathrm{K}}=1 / R_{\mathrm{K}}=\left(\kappa_{\mathrm{ZnO}_{\mathrm{x}} / \mathrm{HQ}} n\right) / d$, where $n$ is the number of inorganic/organic/inorganic interfaces and $d$ is the total thickness of the hybrid films. To reiterate, this formulation of $1 / R_{\mathrm{K}}$ implies that the resistance due to the individual $\mathrm{ZnO} / \mathrm{HQ}$ interfaces and the intrinsic resistance of the organic molecules comprising the interface are lumped as a single resistor.

Figure $4.10 \mathrm{a}$ shows the mean thermal conductance for $\mathrm{ZnO} / \mathrm{HQ} / \mathrm{ZnO}$ interfaces as a function of the inorganic layer thickness (hollow squares). There are two interesting aspects for the conductance calculations shown in Fig. 4.10a. First, the values of the mean conductances for these SLs among the various samples are agreeable within the uncertainties, regardless of the $\mathrm{ZnO} / \mathrm{HQ} / \mathrm{ZnO}$ interface density. This suggests that the series resistor model used to derive these conductances is applicable for our hybrid SLs with single HQ layers, and the previous assumption and discussion regarding fully thermalizing (i.e., black) inorganic/organic boundaries is supported. Along with the results for the hybrid SLs, Fig 4.10a also plots the mean conductances derived from thermal conductivity measurements for $\mathrm{W} / \mathrm{Al}_{2} \mathrm{O}_{3}[158]$ and $\mathrm{AlN} / \mathrm{GaN}$ [4] SLs. Contrary to the hybrid SLs, the mean conductances in these inorganic based SLs increase with decreasing period thicknesses. In Ref. [4], this increase in $h_{\mathrm{K}}$ for the AlN/GaN SLs was attributed to phonons with long wavelengths carrying the majority of heat.

The second aspect is that the mean conductances derived are close to the maximum conductance with $\zeta=1$. This consistency is demonstrated over a wide range of temperatures, shown in Fig. 4.10 p, which plots $h_{\mathrm{K}}$ calculated for $\mathrm{ZnO} / \mathrm{HQ} / \mathrm{ZnO}$ interface as a function of temperature for the two SLs with $\mathrm{x}=13.1 \mathrm{~nm}$ and $7.0 \mathrm{~nm}$. The appreciable agreement between these values and the conductance in $\mathrm{ZnO}$ is consistent with the analysis in Fig. 4.9 (treating all phonon mean free paths being limited by scattering at the $\mathrm{ZnO} / \mathrm{HQ} / \mathrm{ZnO}$ interface), as mentioned above. This agreement also suggests that a large portion of the phonon modes in the $\mathrm{ZnO}$ transmits ballistically across the $\mathrm{ZnO} / \mathrm{HQ} / \mathrm{ZnO}$ interface, implying relatively minor intrinsic thermal resistance in the molecular layer. While the relatively minor disagreement between the maximal conductance (Fig. 4.10p, solid line) and the data could 
imply some level of phonon-vibron interactions in the HQ layer, more rigorous computational models are necessary to draw quantitative conclusions regarding these diffusive scattering processes in the molecule.

In order to quantify the contribution of the vibrational properties of the organic layer on phonon transmission across the $\mathrm{ZnO} / \mathrm{HQ} / \mathrm{ZnO}$ interfacial region, the average phonon transmissions from the results in Fig. 4.10 (comparing the maximal conductance model to the data) are calculated and interfacial transmissions of $\sim 76 \%$ for the $(\mathrm{ZnO})_{7.0} \mathrm{~nm} / \mathrm{HQ}$ sample and $\sim 65 \%$ for the $(\mathrm{ZnO})_{13.1 \mathrm{~nm}} / \mathrm{HQ}$ sample at room temperature are determined. This deviation from "perfect" transmission of phonons could be due to the fact that heat flux carried by phonons with wavelengths longer than the organic molecular chain lengths is unaffected by the organic layer, whereas phonons with wavelengths on the order of and smaller than the molecular lengths are scattered due to the vibrational properties of the molecules.

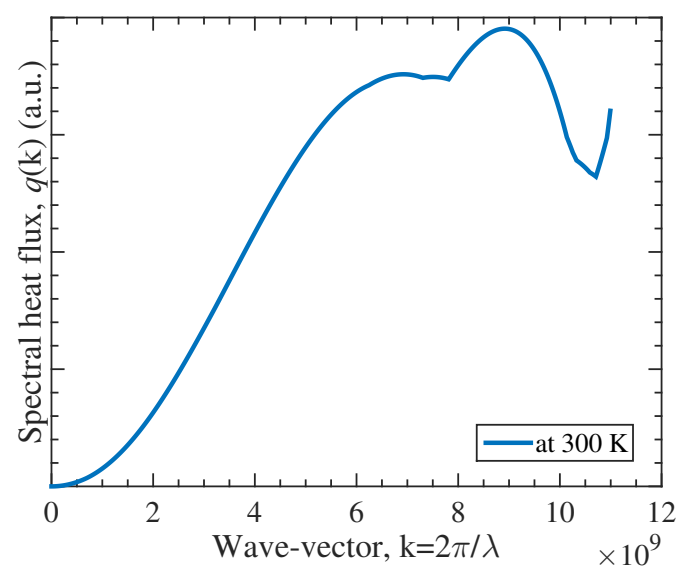

Figure 4.11: Spectral heat flux in $\mathrm{ZnO}$ as a function of the wave-vector in the $\Gamma \rightarrow \mathrm{M}$ direction.

In order to study the role of the vibrational properties of the organic layers contributing to the less than unity transmission coefficient, the spectral heat flux in the $\mathrm{ZnO}$ layer is calculated as a function of the wave-vector of phonons in the $\Gamma \rightarrow \mathrm{M}$ direction (Fig. 4.11). The spectral heat flux provides quantitative measurements of the percent heat flux car- 
ried by different wavelength phonons. The length of the hydroquinone molecules used as the organic constituent are $\sim 6-7 \AA$ and therefore, it is hypothesized that phonons with wavelengths greater than the length of the organic molecule chain are unaffected by the vibrational properties of the molecules, whereas those with wavelengths less than the length of the molecule chains are scattered similar to the analogy of photons in an absorptive media (Beer- Lambert law). The calculations demonstrate that $\sim 9-25 \%$ of the heat flux is carried by phonons with wavelengths shorter than the length of the organic molecules ( 6-7 $\AA$ ). This result is consistent with the high transmission coefficients (average values of $\sim 76 \%$ for the $(\mathrm{ZnO})_{x=7.0 \mathrm{~nm}} / \mathrm{HQ}$ sample and $\sim 65 \%$ for the $(\mathrm{ZnO})_{x=13.1 \mathrm{~nm}} / \mathrm{HQ}$ sample for the 78-300 K temperature range) measured from the TDTR measurements. Note, the transmission did not show any significant temperature dependency within the experimental uncertainty in the results. It should also be noted that this hypothesis is consistent with previous works suggesting that at interfaces, the transmission of phonon wavelengths greater than the characteristic length scales of nanoscale structures and asperities at solid interfaces are not affected by these non-idealities [4, 13, 125, 159-161]. More rigorous computational simulations are necessary to study this hypothesis in more detail, which includes a greater understanding of diffusive vibrational scattering in single-molecule thick films as previously mentioned.

To scope the generality of the discussions presented above, the conductance across the $\mathrm{TiO}_{2} / \mathrm{HQ} / \mathrm{TiO}_{2}$ is derived from the thermal conductivity measurement for the $\mathrm{TiO}_{2}$-based SL at room temperature [137] and is compared to the value obtained for a control sample without the HQ layers $\left(\mathrm{Al} / \mathrm{TiO}_{2} / \mathrm{MgO}\right)$. The values for the conductance for $\mathrm{TiO}_{2} / \mathrm{HQ} / \mathrm{TiO}_{2}$ interface is $h_{\mathrm{K}}=430 \pm 78 \mathrm{MW} \mathrm{m}^{-2} \mathrm{~K}^{-1}$, which is lower than that of the ZnO-based SLs at room temperature. From this, the phonon transmission across the $\mathrm{TiO}_{2} / \mathrm{HQ} / \mathrm{TiO}_{2}$ interface is determined to be $\sim 41 \%$. In this context, $\sim 60 \%$ of the thermal energy in $\mathrm{TiO}_{2}$ is carried by phonons with wavelengths shorter than the mean thicknesses of the HQ layers.

One of the factors driving the high phonon transmission values across $\mathrm{ZnO} / \mathrm{HQ} / \mathrm{ZnO}$ interfaces could be due to the high quality interfaces within the SLs and the precise control 
over the thicknesses of the inorganic layers achieved via the layer-by-layer deposition of the ALD/MLD technique [137, 146]. At the inorganic/organic interfaces, it has been shown through first principles study that the HQ molecules are most probably attached to every other surface $\mathrm{Zn}$ site (50\% surface coverage) [162]. This implies that we can not rule out the possibility of $\mathrm{ZnO}$ growth at the lateral interstitial positions, which could affect the phonon energy transmission across these inorganic/organic/inorganic interfaces; in principle this could happen if the physical size of the HQ would prevent its reaction with all the diethyl zinc terminated surface sites. However, the systematically lower densities shown in Table 4.1 with increasing number of MLD cycles, suggest that the presence of interstitial $\mathrm{ZnO}$ within the organic layers is unlikely.

The implication of large thermal transmission across the single HQ layer assumes that there is no mismatch of acoustic impedance or vibrational spectra encountered by the impinging $\mathrm{ZnO}$ flux on the $\mathrm{HQ}$ monolayer. Although this would be true for a pure $\mathrm{ZnO} / \mathrm{ZnO}$ interface in which phonons are specularly scattered, this clearly would not be the case if considering phonon thermal conductance limited by transmission across the $\mathrm{ZnO} / \mathrm{HQ} / \mathrm{ZnO}$ interface due to properties of the HQ.

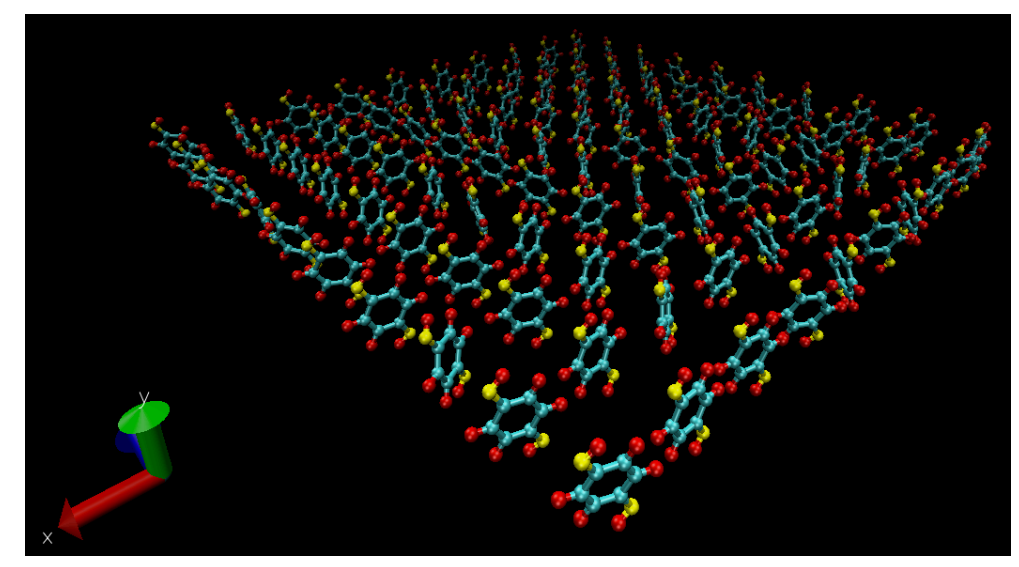

Figure 4.12: Schematic of the MD computational domain with 1400 atoms representing the HQ monolayer interspersed between the inorganic layers. The green atoms are carbon, yellow atoms are oxygen and the red atoms are hydrogen.

To exemplify this more quantitatively, molecular dynamics simulations using the 


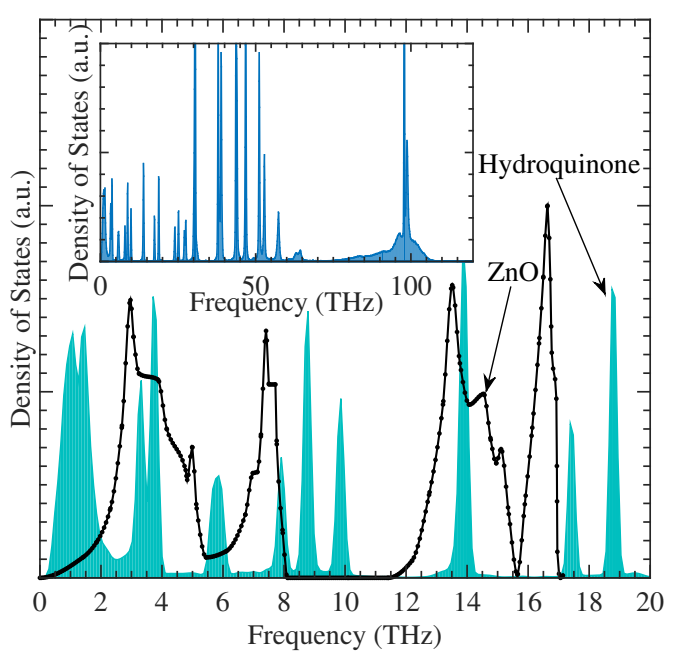

Figure 4.13: Comparison of vibrational density of states for a HQ layer (obtained via molecular dynamics) and $\mathrm{ZnO}$ (obtained from phonon dispersion in Ref. [154]). (inset) The complete local phonon density of states for the HQ layer showing the discrete spikes at a range of phonon frequencies.

LAMMPS package [65] are performed to compute the local phonon density of states in a monolayer of HQ molecules. The schematic of the MD domain containing 1400 atoms is shown in Fig. 4.12, The ReaxFF interatomic potential is used to describe the interactions between the atoms in the HQ molecules [163]. Periodic boundary conditions in the X-and z-directions are prescribed and the shrink-wrap boundary condition on the y-direction is used. The top and bottom oxygen and hydrogen atoms in the y-direction are held fixed so as to replicate the conditions in a monolayer of MLD-grown HQ molecules constrained in between the inorganic layers. The power spectral density is compared to the $\mathrm{D}(\omega)$ spectrum for bulk $\mathrm{ZnO}$ calculated from the phonon dispersion [154] and as expected, the relatively discrete modes in the phonon frequencies calculated for the HQ layer do not completely overlap the $\mathrm{D}(\omega)$ for $\mathrm{ZnO}$ (Fig. 4.13). Note, no attempt to separate the scattering at the $\mathrm{ZnO} / \mathrm{HQ}$ boundary from the internal scattering within the organic monolayers from the MD simulations is made as this is beyond the scope of this study. However, from the predicted phonon density of states for a confined HQ layer mimicking a 50\% surface coverage, it can be inferred that under the typical DMM picture of phonon transmission from the $\mathrm{ZnO}$ 
across the HQ and into the next $\mathrm{ZnO}$ layer, a HQ-limited transmission seems implausible.

\subsection{Effect of the type and thickness of the organic layer on thermal transport}

This section investigates the effect of increase in the thickness of the HQ layers (achieved through multiple MLD cycles in between the ALD cycles) as well the effect of incorporating a different organic layer in between the inorganic $\mathrm{ZnO}$ layers. For the latter purpose, thin films consisting of alternating layers of zinc oxide and TEMPO-oxidized cellulose nanofibers are fabricated using a combination of atomic layer deposition and dip coating to form an inorganic-organic hybrid superlattice structure. Both, increasing the HQ thicknesses and using nanofibers instead of HQ are shown to further reduce the thermal conductivities.

In order to investigate the role of molecular vibrations on the phonon scattering mechanisms, the thermal conductivities were measured for SLs with 3, 5 and 7 layers of HQ molecules interspersed between $\mathrm{x}=7.0 \mathrm{~nm}$ thick $\mathrm{ZnO}$ layers at room temperature (see top panel of Fig. 4.14 for depictions of unit cells [162]). As shown in Fig. 4.14a, increasing the number of MLD cycles for the SLs decreases the thermal conductivity monotonically. Note, the prediction of Eq. 4.4 for $\mathrm{x}=7 \mathrm{~nm}$ does not agree with the measurements for thicker HQ layers, which is ascribed to $\mathrm{ZnO}$ phonons scattering in the organic layers due to the vibrational properties of the thicker HQ layers. To further quantify the role of the vibrational resistance on these composite structures, we plot the mean thermal boundary resistance across the $\mathrm{ZnO} / \mathrm{HQ} / \mathrm{ZnO}$ interface as a function of number of molecular layers in Fig. $4.14 \mathrm{p}$ as calculated from the series resistor model. The linear trend in resistance as the HQ layer is increased from 3 to 7 layers suggests that the internal diffusive scattering in the organic layer plays a significant role in impeding thermal transport for SLs with greater than or equal to 3 layers of HQ in-between the inorganic layers. It should be noted that from GIXRD measurements, a significant reduction in the crystallinity of the inorganic 

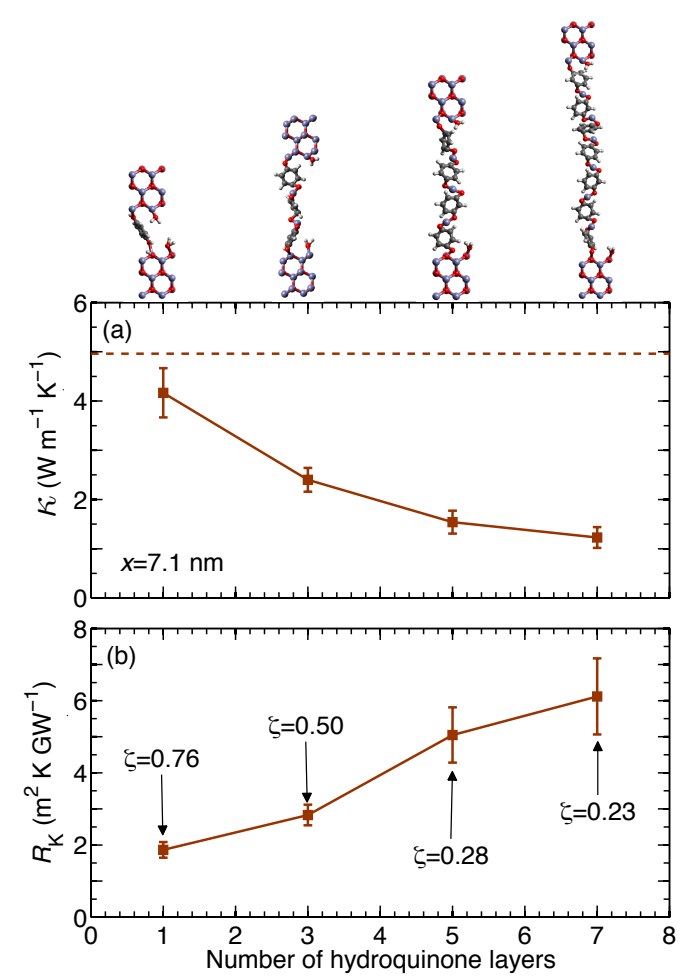

Figure 4.14: Top panel depicts unit cells with increasing number of hydroquinone molecules. (a) Thermal conductivity measurements at room temperature as a function of the number of MLD cycles performed. Calculation of Eq. 4.4 for the inorganic layer thickness is also shown for comparison. The measured thermal conductivity for the SLs deviates from the prediction of Eq. 4.4 as the HQ layer thicknesses increase. (b) Effective resistances of inorganic/organic/inorganic interfaces with varying number of hydroquinone layers derived from the thermal conductivities shown in (a).

constituents due to inclusion of the thicker HQ layers is not observable, implying that the reduction in thermal conductivities of these structures with 3 to 7 HQ layers is mainly due to scattering at the thicker HQ layers.

To quantitatively support this result, the average phonon transmission from the $\mathrm{ZnO}$ across the HQ layer using the approach discussed previously (transmissions shown in Fig. 4.14b) is calculated. Increasing the MLD cycles from a monolayer to 3 HQ layers drastically reduces the transmission from $76 \%$ to $53 \%$. Upon further increase in the number of HQ layers to 5 and 7 , the transmissions reduce to $28 \%$ and $23 \%$, respectively. Previous studies on the length dependent vibrational transport in molecular chains have 
Table 4.2: Thermal conductivity values measured for the $(\mathrm{ZnO})_{\mathrm{x}} / \mathrm{TOCN}$ hybrids films.

\begin{tabular}{lc}
\hline \hline Number of organic layers & $\kappa\left(\mathrm{W} \mathrm{m}^{-1} \mathrm{~K}^{-1}\right)$ \\
\hline 3 & $3.63 \pm 0.27$ \\
5 & $2.74 \pm 0.18$ \\
9 & $1.94 \pm 0.14$ \\
\hline \hline
\end{tabular}

mostly focused on self assembled monolayers of aliphatic alkane chains [142, 164-166]. Most of these studies have concluded that the conductance across molecular chains is insensitive to the length of the hydrocarbon chains, particularly in Ref. [166], it is shown that the conductance is constant for chain lengths $>20$ carbon atoms. However, for shorter chain lengths, theoretical calculations by Segal et al. [166] and experimental data by Meier et al. [165] suggest that conductance is maximum for a chain length of up to 4 carbon atoms and decreases with increasing number of carbon atoms thereafter to a certain chain length. From the results, the drastic reduction in phonon transmission coefficients with thicker HQ layers compared to that of the SLs with a monolayer of HQ molecules could be due to the diffusive nature of vibrational transport in the longer chain molecules. However, as pointed out previously, it is not possible to comprehensively separate the resistances due to inorganic/organic interface scattering and the internal scattering in the molecular layers.

The MLD technique used for the fabrication of hybrid structures with HQ layers is not ideal for depositing polymer layers in between the inorganic layers. Therefore, dipcoating is utilized to deposit TEMPO-oxidized cellulose nanofibers (TOCN) as the organic layers to gauge the effect of using an organic material with a vastly different structure compared to the HQ molecules. More specifically, unlike the aromatic structure of the HQ, the nanocellulose layers are made up of glucan chains of crystalline cellulose microfibrils.

A sample set with 3, 5 and 9 layers of TOCN were dip-coated in between $\mathrm{ZnO}$ layers to form multilayer films with $100 \mathrm{~nm}$ thickness. The sample fabrication and characterization is detailed in Ref. [167]. The measured thermal conductivity values are listed in Table 4.2 . The $(\mathrm{ZnO})_{\mathrm{x}} / \mathrm{TOCN}$ films display large reductions in their thermal conductivity of more 
than an order of magnitude compared to that of a $\mathrm{ZnO}$ thin film, and the effect becomes larger as the number of cellulose nanofiber layers is increased. This is expected, since the total number of $\mathrm{ZnO}$ ALD cycles in the films was kept constant at 600 , so as the number of organic layers increases, the separation between individual TOCN layers decreases; more specifically, the period thickness changes from $25 \mathrm{~nm}$ in the $\mathrm{N}=3$ film to $10 \mathrm{~nm}$ in the $\mathrm{N}$ $=9$ sample. Thus, not only were there more interfaces to scatter phonons in the films with higher TOCN content, the layer thicknesses of the inorganic constituents start to become more comparable to the intrinsic phonon mean free paths in zinc oxide.

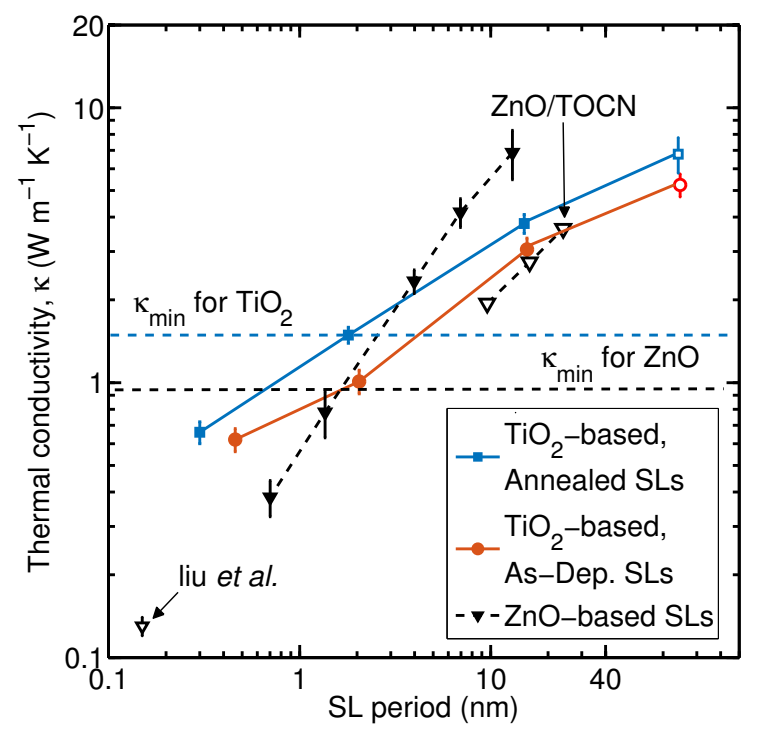

Figure 4.15: Thermal conductivities of $\mathrm{ZnO}$-and $\mathrm{TiO}_{2}$-based SLs as a function of SL period thickness. The measured thermal conductivity for a $k: m=1: 1$ hybrid film reported in Ref. [44] is also plotted (hollow triangle). The calculated minimum in thermal conductivities for $\mathrm{ZnO}$ and $\mathrm{TiO}_{2}$ are also shown for comparison. Note, for the case of as-deposited and annealed $\mathrm{TiO}_{2}$, the SL period of $\sim 100 \mathrm{~nm}$ are for the corresponding purely ALD grown samples with $k=0$ (hollow symbols).

In Fig. 4.15, the measured thermal conductivities as a function of SL period thickness for the $\mathrm{ZnO}$ - and $\mathrm{TiO}_{2}$-based SLs are shown. Compared to the $\mathrm{ZnO} / \mathrm{HQ}$ hybrid films, the $\mathrm{ZnO} / \mathrm{TOCN}$ films have lower thermal conductivities for the same period spacings. In this context, the TOCN layers are more effective in reducing the thermal conductivity due to the thicker size of the cellulose nanofibers formed during dip-coating as compared to the 
MLD-grown HQ monolayers. Regardless of the organic material used, there is a strong correlation between the thickness of the organic layer and the thermal conductivity of the hybrid superlattice film. With more than an order of magnitude difference in the measured thermal conductivities, hybrid multilayers in general demonstrate a wide range of tunability in the design of their thermal conductivities.

The results shown in Fig. 4.15 demonstrate that as the SL period thickness increases, the thermal conductivities increase monotonically for all the hybrid SLs. The drastic increase in $\kappa$ as a function of SL period thickness of the ZnO-based SLs as compared to the $\mathrm{TiO}_{2}$-based SLs is due to the fact that the phonon flux in a $\mathrm{ZnO}$ layer is much greater than that in the $\mathrm{TiO}_{2}$ layer [126], as discussed above. Furthermore, the $m=4 \mathrm{TiO}_{2}$-based SLs and the $m=9$ and $4 \mathrm{ZnO}$-based SLs demonstrate thermal conductivities that are lower than the theoretical minimum, further providing evidence that thermal transport in these SLs is severely limited by the SL period thicknesses. Figure 4.15 also includes the thermal conductivity measured via TDTR for a ZnO-based ALD/MLD grown thin film with $k: m=1: 1$ [44]. We estimate the period spacing for the $\mathrm{ZnO}$ layers in their structure to be $\sim 0.15 \mathrm{~nm}$, which is a reasonable estimation considering that the average growth rate reported in Ref. [44] is $0.15 \mathrm{~nm} /$ cycle. Their measured thermal conductivity is in line with the decreasing trend in the thermal conductivity with decreasing period spacing for our hybrid samples.

\subsection{Heat capacity measurements}

Figure 4.16 shows the measured volumetric heat capacities for the $\mathrm{TiO}_{2}$ - and $\mathrm{ZnO}-$ based SLs as a function of temperature. Along with the thermal conductivities, the heat capacities of the $\mathrm{TiO}_{2}$-based SLs increase significantly due to the high annealing treatment as shown in Fig. 4.16a. For comparison, the bulk heat capacities of $\mathrm{TiO}_{2}$ are also shown [168]. As expected, the heat capacities of the as-deposited SL are close to the values for the bulk heat capacities due to the fact that the fraction of the organic component in the SL 
film is relatively small (the SL is fabricated with 40 ALD cycles for every 1 MLD cycle). Similarly, the measured heat capacities for the ZnO-based sample (with $m=9$ ) agree very well with the bulk $\mathrm{ZnO}$ heat capacities (Fig. 4.16p) [169].

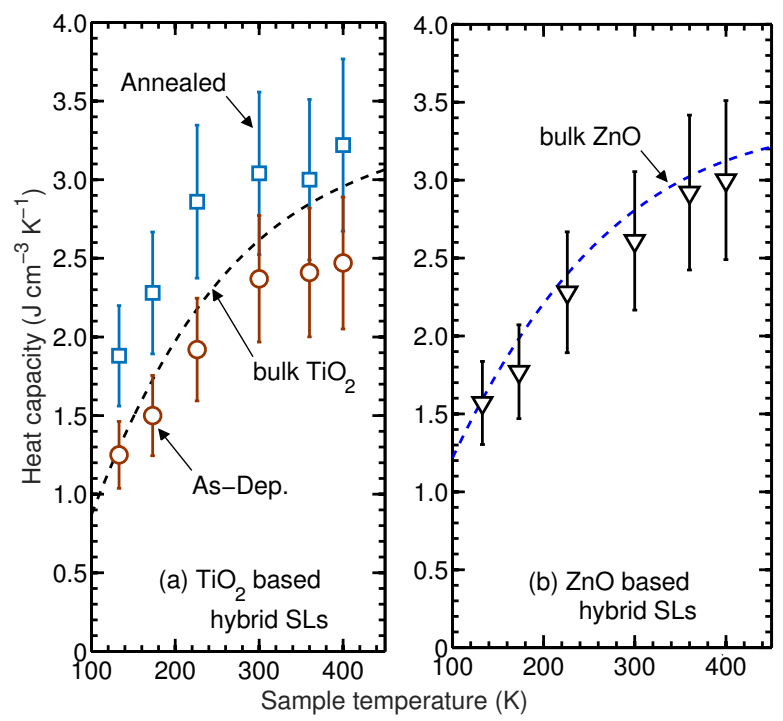

Figure 4.16: Measured volumetric heat capacities as a function of temperature for (a)

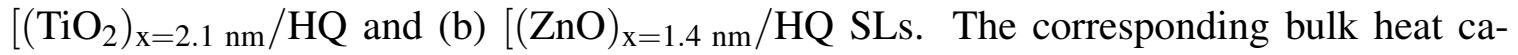
pacities for the inorganic constituents are also shown for comparison [168, 169].

To understand the effect of higher fractions of organic constituents on the heat capacity of the hybrid SLs, the results for the SLs with varying number of organic monolayers as a function of their densities are compared in Fig. 4.17 a. The conversion from a HQ monolayer to a 2D graphitic layer results in the decrease of the thicknesses of the annealed samples, as mentioned earlier. As a result, the densities of the annealed samples increase considerably (as determined from XRR measurements of the thin films). Since the volumetric heat capacity of a substance is directly proportional to mass density, therefore, the volumetric heat capacity plotted in Fig. $4.17 \mathrm{~A}$ increases monotonically for the as-deposited samples as the density increases. The inclusion of more HQ in the SLs decreases the volumetric heat capacity in general due to the reduction in density with higher MLD cycles. However, the volumetric heat capacities of the annealed samples (with the 2D graphitic layers) are greater than the as-deposited samples as well as that of the corresponding inorganic 
bulk constituent for the $\mathrm{TiO}_{2} \mathrm{SLs}$, as mentioned in the previous paragraph.
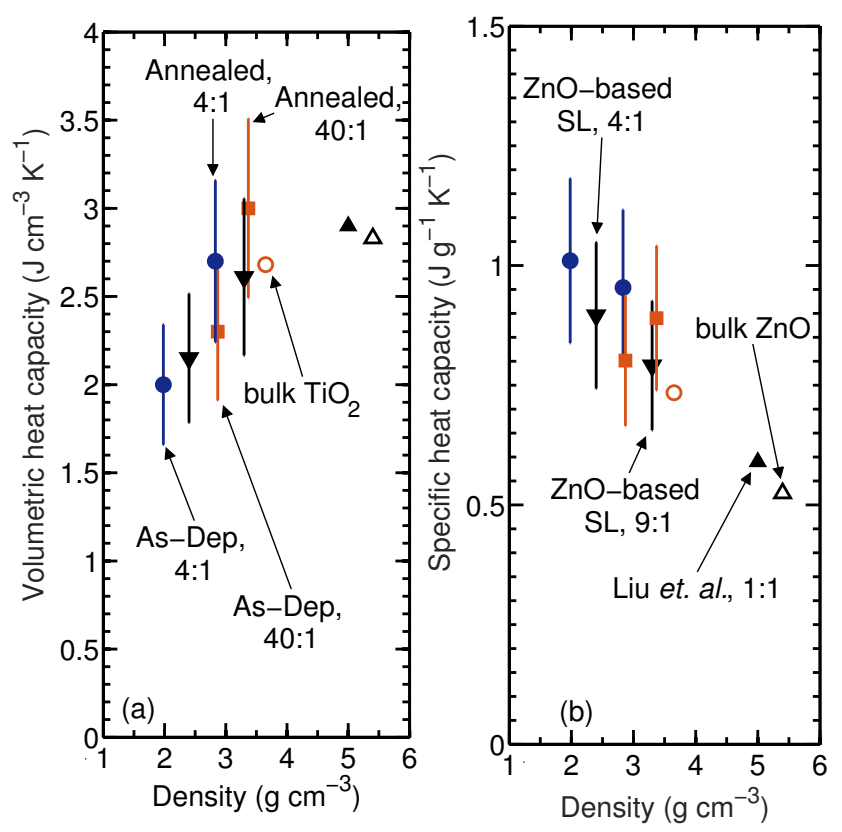

Figure 4.17: (a) Volumetric heat capacities of the $\mathrm{TiO}_{2}$ - and $\mathrm{ZnO}$-based thin films plotted as a function of density along with the values of bulk heat capacities for the corresponding inorganic constituents. (b) Specific heat capacities plotted as a function of density. In these plots, the data are designated as follows: $\mathrm{TiO}_{2}$ (circles), $\mathrm{ZnO}$ (triangles), corresponding bulk (open symbol), ALD/MLD SLs (closed symbols). For comparison, the heat capacity measured by Liu et al. [44] for a $k: m=1: 1 \mathrm{ZnO}$-based hybrid film is also plotted in the figures.

Figure 4.17p shows the measured specific heat capacities of the materials calculated by dividing the measured volumetric heat capacities by their respective measured mass densities (hence, the specific heat capacity is defined based on mass, where the specific heat capacity, $c$, is given by $c=C / \rho$, where $\rho$ is the mass density). As is expected, the specific heat capacities of the hybrid SLs deviates from the value of the bulk inorganic constituent as the number of organic monolayers increases. At room temperature, the specific heat of the as-deposited $\mathrm{TiO}_{2}$ - and $\mathrm{ZnO}$-based SLs with $m=4$ is closer to the value of the organic counterpart, i.e., poly-phenylene oxide $\left(1.204 \mathrm{~J} \mathrm{~g}^{-1} \mathrm{~K}^{-1}\right)$. Whereas, the specific heat capacities of the SLs with $m=40$ and $m=9$ for the $\mathrm{TiO}_{2}$ - and $\mathrm{ZnO}$-based SLs, respectively, approach the value of their corresponding inorganic constituents. Unlike, the change in 
the volumetric heat capacities after the high annealing treatment, the specific heat of the annealed SLs show good agreement within uncertainties with their as-deposited counterparts (even though the densities increase with annealing). Figure $4.17 \mathrm{~b}$ also includes the measured specific heat of a $k: m=1: 1 \mathrm{ZnO}$-based hybrid sample reported in Ref. [44] (with a density of $5 \mathrm{~g} \mathrm{~m}^{-3}$ ). Their result matches the decreasing trend in the measured specific heat with increasing mass density as with the hybrid multilayers studied here.

\subsection{Summary}

In summary, the thermal conductivities and heat capacities of $\mathrm{TiO}_{2}$ - and $\mathrm{ZnO}$-based hybrid SLs with periodic organic layers between the inorganic constituents are measured. The inclusion of organic layers is shown to drastically reduce the thermal conductivities of these SLs. Furthermore, the thermal conductivities of these hybrid SLs are shown to scale linearly with increasing period thickness, suggesting that boundary scattering at the inorganic/organic/inorganic interface dominates the heat transfer in these structures. Similarly, the inclusion of organic layers is also shown to decrease the heat capacities. The effect of high temperature annealing treatment on the $\mathrm{TiO}_{2}$-based SLs is shown to increase both the thermal conductivities and heat capacities. The increase in thermal conductivities due to annealing is attributed to enhanced crystallinity (not due to any intrinsic vibrational properties of the organic layer), whereas, the increase in heat capacities is attributed to the increase in the densities of the samples.

The heat transfer mechanisms in hybrid SLs with single molecular layers are driven by phonon-boundary scattering, where the entire spectrum of phonon mean free paths in the inorganic layer is limited by scattering at the inorganic/organic interface. The resulting thermal conductivities of these hybrid nanostructures are mainly limited by the $\mathrm{ZnO}$ phonon flux and period spacing of the inorganic layers. The analysis presented above suggests that the phonon flux in the inorganic layer, which scatters at the inorganic/organic interface, limits the thermal conductivity of these nanostructures. The mean conductances derived 
from the thermal conductivity measurements also suggest that scattering at the molecular layer interfaces accounts for the majority of the reduction in the thermal conductivity of hybrid SLs with single organic layers. By considering this as a thermal boundary conductance limited process, it is hypothesized that phonons with wavelengths greater than the organic layer thickness are transmitted across the organic layers after scattering at the inorganic/organic interface; these phonon wavelengths make up $>75 \%$ of the phonon flux in the $\mathrm{ZnO}$, which offers a concomitant picture of the heat transfer processes in inorganic/organic hybrid composites. By increasing the thickness of the MLD-grown layer or using nanocellulose layers intead of the HQ molecules, we observe a significant reduction in the phonon transmission across the thicker molecular layers as compared to the thermal conductance across HQ monolayers. The linear trend in thermal resistance with number of molecular layers suggests a diffusive scattering process in the MLD-grown organic layer, which offers a robust opportunity for more focused theoretical or computational studies to pinpoint the size effects in vibronic scattering in aromatic molecules. 


\section{Chapter 5}

\section{Thermal transport across}

\section{amorphous-based confined thin films}

\section{and superlattices}

In the previous chapter, monolayer thin organic layers were shown to drastically reduce the thermal conductivity of inorganic crystalline films. In this chapter, thermal transport in various geometries of disordered inorganic interfaces is investigated. Specifically, this chapter studies the thermal transport across interfaces in amorphous-based superlattices and disordered confined films between crystalline leads. It is found that the resistances at a single amorphous/crystalline interface or amorphous/amorphous interface in these structures are much lower than those at interfaces between the corresponding crystalline materials, suggesting that diffusive scattering at an interface could result in higher energy transmissions in these systems. Both computational and experimental results on amorphous-based superlattices are presented to support this hypothesis. The results of these studies, together with the fact that high mass ratios between amorphous and crystalline materials can lead to higher thermal resistances across thin films, are used to design amorphous/crystalline superlattices with very low thermal conductivities. In this regard, the thermal conductivities of amorphous/crystalline superlattices are predicted via MD and it is demonstrated that the 
thermal conductivities decrease monotonically with increasing interface densities above 0.1 $\mathrm{nm}^{-1}$, similar to the results for amorphous/amorphous superlattices. These thermal conductivities are lower than that of the homogeneous amorphous counterparts, which alludes to the fact that interfaces non-negligibly contribute to thermal resistance in these superlattices. Another interesting finding from the MD simulations is that the thermal conductivity of superlattices can be reduced below the amorphous limit of its material constituent even when one of the materials remains crystalline. This has implications to thermoelectric materials where crystallinity is desirable for higher electronic mobilities. Major portions of this chapter appeared in two publications in the Journal of Applied Physics: "Effect of crystalline/amorphous interfaces on thermal transport across confined thin films and superlattices" [170] and "Kapitza resistance and the thermal conductivity of amorphous superlattices" [171].

\subsection{Introduction}

In practical applications that utilize sputtering or evaporation techniques resulting in non-epitaxial film deposition, disorder and film oxidation at the interfacial layer between two solids (e.g., metal-oxide-semiconductor (MOS) structures containing silicon include a non-stoichiometric oxide layer present at the $\mathrm{Si} / \mathrm{SiO}_{2}$ interface [172]) can introduce additional resistance to thermal transport. This disruption of crystallinity in thin films can influence the material's vibrational characteristics, which in turn influences the thermal boundary resistance (TBR) across the thin films. Note, throughout this chapter, we refer to the TBR across thin interfacial films as a lumped resistance that takes into account resistances at both film/lead boundaries and the resistance due to the interfacial film layer. Furthermore, TBR is used throughout this chapter as opposed to TBC, which was used in all the previous chapters. The reason being that several references are made to series resistance analyses, so referencing interfacial thermal transport to TBR as opposed to TBC will avoid confounding language in the discussions hereafter. 
Computationally, the study of thin films acting as interfacial layers between two solids has demonstrated the importance of film thickness and vibrational bridging (between the leads) on TBR. English et al. [173] showed that tuning the vibrational properties of an interfacial layer between two dissimilar materials can decrease the TBR. Landry and McGaughey [174] showed that when the length scales of the interfacial thin film are on the order of the intrinsic phonon mean free paths, ballistic phonon transport can reduce the resistance across these crystalline interfacial layers such that the often employed thermal circuit model fails to capture this phenomenon. Taken together, the role of the interfacial layer's length, intermixing between the leads, and mass-mismatch with the leads can greatly influence the total TBR across confined thin films; manipulating these properties can provide useful tunability in the overall thermal transport across devices with ordered or disordered interfacial layers.

The aforementioned computational studies have mainly focused on confined crystalline films with atomically smooth interfaces. These "perfect" interfaces are generally not present in real world applications (e.g., MOS structures, which inherently include an amorphous native oxide interfacial layer). Therefore, one of the main goals of this study is to understand the role of amorphous interfacial layers and the effect of mass and thickness of these layers on the TBR. Generally speaking, pristine interfaces are considered ideal for interfacial heat flow and diffusive scattering caused by disruption of crystallinity in the vicinity of interfaces is usually thought to impede thermal transport. In this regard, the contribution of the individual lead/film resistances on the total TBR across amorphous interfacial layers will be investigated.

Along with the individual resistances at individual lead/film boundaries (which are associated with crystalline/amorphous interfaces), this chapter also seeks to understand TBR across amorphous/amorphous interfaces. For this purpose amorphous-based SLs will be investigated both computationally and experimentally. The electrical and optical properties of amorphous-based semiconductor superlattices (SLs) have been a subject of scientific inquiry since Abeles and Tiedje first provided evidence that the SLs exhibit quantum 
size effects [175]. In addition, amorphous SLs provided a platform for some of the earliest experimental observations of the coherent characteristics of lattice vibrations [176], including the formation of zone-folded acoustic modes [177] and phonon stop bands [178]. These vibrational modes in amorphous and disordered solids have been described using a different taxonomy compared to crystalline materials due to the lack of periodicity in their atomic arrangement [179]. Unlike in crystalline solids, vibrations in amorphous and disordered materials are classified as propagons (that are delocalized, propagating modes), diffusons (that are non-propagating, delocalized modes) and locons (that are localized and non-propagating modes) [180]. While locons do not contribute to the thermal conductivity, diffusons mediate heat through harmonic coupling of localized modes [181, 182].

In amorphous-based SLs, even though the propagating low frequency vibrations are affected by the artificial periodicity due to their extensive coherence lengths, it is generally assumed that the amorphicity within each layer should dictate the properties of higher frequency, non-propagating vibrations (and hence, thermal transport) [183]. Under this assumption, interfaces within amorphous SLs would not provide any additional thermal resistance. This perspective was supported by the report of Zhang et al. [45] where the measured thermal diffusivities of a-Si:H/a-SiN ${ }_{\mathrm{X}}: \mathrm{H}$ SLs were well described by effective medium theory when the interfaces within the SLs were ignored. Therefore, along with amorphous confined thin films, NEMD simulations are implemented to study the role of TBR on the vibrational thermal transport in amorphous-based SLs. Furthermore, SLs composed of hydrogenated amorphous silicon carbide $(\mathrm{a}-\mathrm{SiC}: \mathrm{H})$ and hydrogenated silicon oxycarbide (a-SiOC:H) are studied via the TDTR technique to support the computational results and shed more light on the thermal transport across amorphous/amorphous interfaces. These material systems are investigated due to their unique characteristics that are of technological and scientific importance, specifically as low- $k$ dielectric materials [184-186].

Finally, based on the results from the amorphous confined thin films and the amorphousSLs, this chapter also seeks to design amorphous/crystalline SLs with high interface densities where the resistances across the interfaces contribute to lowering the thermal conduc- 
tivities of these structures (even beyond that of the amorphous counterpart).

\subsection{Disordered interfacial thin films}

\subsubsection{Computational details}

A schematic of the computational domain for confined thin films between crystalline leads is shown in the top panel of Fig. 5.1. These systems are either composed of i) crystalline Si leads with an amorphous or crystalline layer of Ge in-between the leads; or ii) crystalline Ge leads with an amorphous or crystalline layer of $\mathrm{Si}$ in-between the leads. The $\mathrm{Si}-\mathrm{Si}, \mathrm{Ge}-\mathrm{Ge}$ and $\mathrm{Si}-\mathrm{Ge}$ interatomic potentials are described by the Tersoff potential [187, [188], which accounts for the strain associated with the lattice mismatch at a $\mathrm{Si} / \mathrm{Ge}$ interface [188]. All simulations for these systems are performed at an average temperature of 500 $\mathrm{K}$, where elastic scattering has been shown to dominate the TBR [189]. Fixed boundary conditions are applied on the $z$-direction by fixing 4 monolayers of atoms that border the hot and cold reservoirs as shown in the top panel of Fig. 5.1. Periodic boundary conditions are applied in the $x$ - and $y$-directions. The leads are each 400 monolayers and the cross section area $A_{\mathrm{c}}$ of the simulation domain is set to five unit cells by five unit cells. Based on previous MD simulations on similar $\mathrm{Si} / \mathrm{Ge}$ systems, size effects are not expected to affect these MD-predicted TBRs [174, 189]. A time step of $0.5 \mathrm{fs}$ is applied throughout the simulations and the structures are initially equilibrated with an isothermal-isobaric ensemble (NPT integration with the number of particles, pressure and temperature of the system held constant)[66] at zero-pressure and $500 \mathrm{~K}$ for a total of 2.5 million time steps.

To determine the TBR across the confined amorphous and crystalline films, the NEMD method is implemented where a thermal flux, $q$, is applied across a computational domain to establish a steady-state temperature gradient, $\partial T / \partial z$. During the implementation of a heat flux, the thermostat is removed and the simulation is performed under the NVE integration (with number of particles, volume and energy of the system held constant), which adds and removes a fixed amount of energy per time step from warm and cool baths, respectively. In 

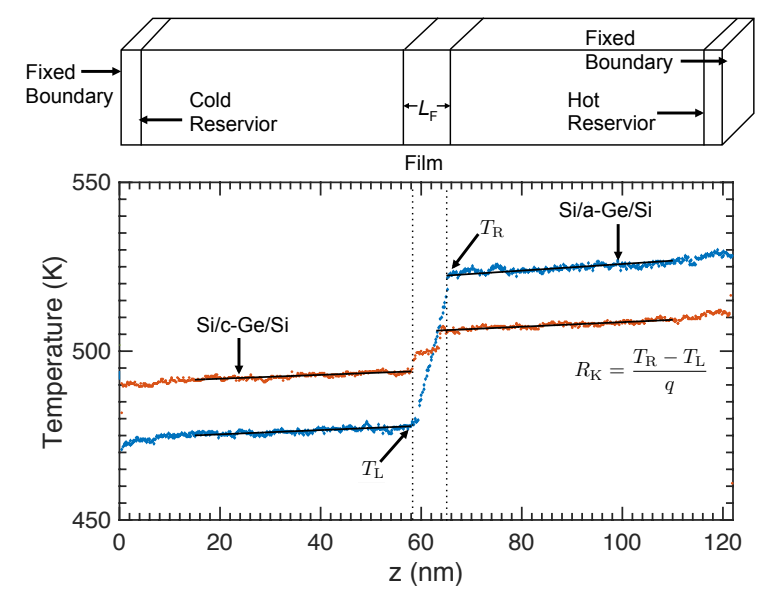

Figure 5.1: (Top panel) Schematic of the simulation cell for thin films confined between crystalline leads. Thermal flux is applied across the simulation cell in the $z$-direction. (Bottom panel) Temperature profile of $\mathrm{Si} / \mathrm{Ge} / \mathrm{Si}$ systems with disordered and crystalline $\mathrm{Ge}$ films.

doing so, a thermal flux of $q=3.0 \mathrm{GW} \mathrm{m}^{-2}$ is applied across the computational domains; note, increasing $q$ by $50 \%$ produced statistically invariant TBRs for the $\mathrm{Si} / \mathrm{Ge}$-based structures. After the initial application of the heat flux, we let the system reach steady-state for 3 ns. The steady-state temperature profile is determined by averaging the temperature of the computational domain (by dividing it into equally spaced bins) for an additional $3 \mathrm{~ns}$. From the temperature profiles, the TBRs across the interfacial films are determined by the relation, $q=R_{\mathrm{K}}^{-1} \Delta T$ as shown in the bottom panel of Fig. 5.1. To determine the temperature drop at the film boundaries, linear regression analyses to the temperature profiles of the leads are applied and the exact temperatures at the boundaries between the leads and the film are calculated from the linear fits to the MD-data (Fig. 5.1). The linear fits to the temperature profiles reduce the uncertainty associated with determining the exact temperature at the boundaries; this approach has been used previously to determine interfacial resistances across solid-solid [12, 173, 174, 189, 190] solid-liquid [191, 192] and solid-gas [193-195] systems. 


\subsubsection{Thermal boundary resistance across interfacial thin films}

Figure 5.2 shows the MD predicted TBRs across the amorphous and crystalline confined films (for the $\mathrm{Si} / \mathrm{Ge} / \mathrm{Si}$ and $\mathrm{Ge} / \mathrm{Si} / \mathrm{Ge}$ systems) as a function of the film thickness, $L_{\mathrm{f}}$. The MD simulations are carried out for films with thicknesses between 2 and $25 \mathrm{~nm}$. Films with thicknesses $<2 \mathrm{~nm}$ are not considered in this study due to the fact that the allowed vibrational states highly depend on the length of the films below $2 \mathrm{~nm}$; in these cases, the TBRs increase rapidly with increasing thickness of the confined films until $2 \mathrm{~nm}$ for both Si and Ge films [174, 196]. In this context, only the thickness regime where the material's intrinsic vibrational density of states does not depend on the thickness of the film is investigated.

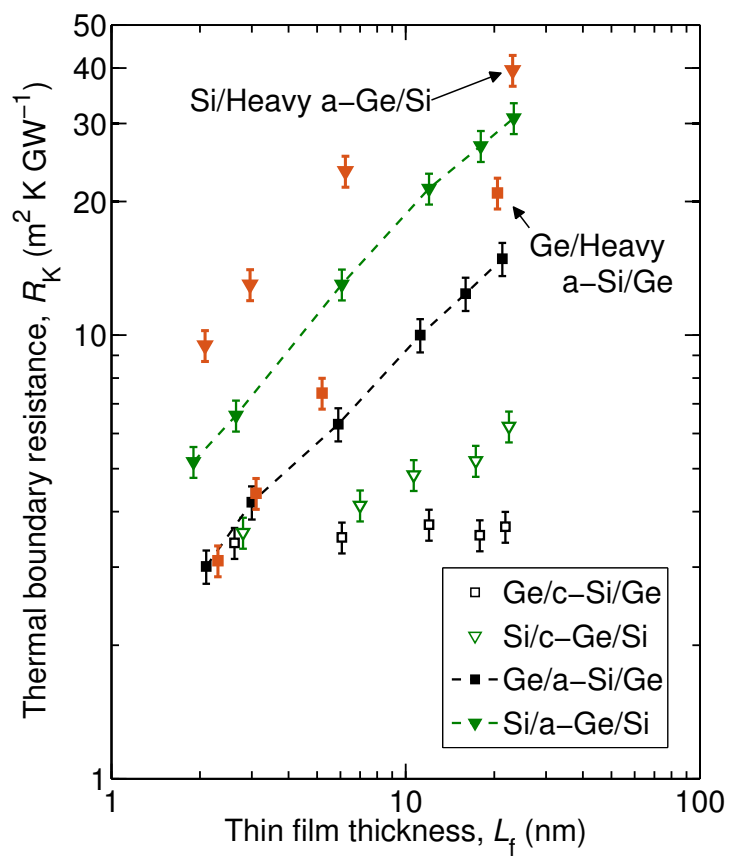

Figure 5.2: Thermal boundary resistances across the confined thin films predicted via NEMD simulations performed at $500 \mathrm{~K}$ temperature. The TBRs across Ge/c-Si/Ge are represented as hollow square symbols, solid square symbols represent Ge/a-Si/Ge, hollow green triangles represent $\mathrm{Si} / \mathrm{c}-\mathrm{Ge} / \mathrm{Si}$ and solid green triangles represent $\mathrm{Si} / \mathrm{a}-\mathrm{Ge} / \mathrm{Si}$. For the amorphous thin films, the TBRs for interfacial layers with twice the mass of the layers are also shown. 
For the $\mathrm{Si} / \mathrm{c}-\mathrm{Ge} / \mathrm{Si}$ structures, the TBR increases monotonically with $L_{\mathrm{f}}$ (hollow triangles in Fig. 5.2). In contrast, the resistances across the Ge/c-Si/Ge structures are independent of the film thicknesses studied in this work (hollow squares). Often, the resistance across interfacial thin films is described by a thermal circuit model, which inherently represents the resistance for the diffusive limit where the film thickness is much greater than the mean-free-path of the phonons in the film. The total resistance calculated by this model is the sum of the two resistances associated with the film boundaries and the resistance due to the finite thickness of the film. However, the validity of this model is limited to situations where non-diffusive transport is negligible. When comparing the MD-predicted resistances across the Ge/c-Si/Ge films for all thicknesses and for films $<20 \mathrm{~nm}$ for the $\mathrm{Si} / \mathrm{c}-\mathrm{Ge} / \mathrm{Si}$ structures, the thermal circuit model grossly overpredicts MD simulated resistances [174]. This is due to the fact that the heat carrying phonons have mean-free-paths greater than or on the order of the thickness of the thin films and therefore the diffusive transport description fails to replicate the MD predictions due to considerable size effects. This deviation from the diffusive limit is more apparent in the Ge/c-Si/Ge structures where a change in the TBR is not observed with increasing film thicknesses from $2 \mathrm{~nm}$ to $20 \mathrm{~nm}$ due to the fact that the phonon transport across the thin films is mostly ballistic [174]. This is also evident from the increase in the thermal conductivities $(\sim 6$-folds $)$ predicted from the temperature profiles of the thin films compared to the bulk value of $\mathrm{Si}$. In contrast, for the $\mathrm{Si} / \mathrm{c}-\mathrm{Ge} / \mathrm{Si}$ structures, the thermal resistance for thicker films increases monotonically as shown in Fig. 5.2, suggesting that phonon-phonon coupling becomes prominent for these films. These results are consistent with the NEMD predictions by Landry and McGaughey [174] on lattice matched SW-based Si/Ge structures where they compare their MD-predictions to that of lattice dynamics calculations and conclude that phonon transport is mostly ballistic for the $\mathrm{Ge} / \mathrm{c}-\mathrm{Si} / \mathrm{Ge}$ and more diffusive for the $\mathrm{Si} / \mathrm{c}-\mathrm{Ge} / \mathrm{Si}$ structures.

In comparison to the crystalline films, the TBR for the structures with the amorphous thin films are significantly greater for thicker films as shown in Fig. 5.2. The linear increase in resistance is suggestive of diffusive transport across the amorphous interfacial 
regions. Moreover, the thickness trend in resistances predicted from the thermal circuit model, which only considers the resistance due to the amorphous layer $\left(L_{\mathrm{f}} / k\right)$, agrees well with the MD predictions due to the diffusive nature of heat propagation in the amorphous interfacial regions. Owing to the very low thermal conductivities of the amorphous layers, it is very difficult to accurately determine the resistance across a single amorphous/crystalline interface from the MD predicted temperature profiles for these structures. Typically, for crystalline systems, the distinction between an interfacial resistance and film thermal conductivity is well defined under the NEMD framework due to the distinct temperature discontinuity at interfaces. This distinction across the crystalline/amorphous interfaces in our simulations is not clear, as the temperature discontinuities at these interfaces are minute. However, the accurate prediction of interfacial resistance at these boundaries could be achieved via equilibrium MD simulations where the resistance is calculated based on tracking the equilibrium fluctuations and not dependent on the temperature discontinuity [197, 198]. Previously, this method has been used to predict thermal interface conductance in crystalline/crystalline Si/Ge superlattices [197] and the resistance across a Lennard-Jones based solid-gas interface [199]. In fact, in Ref. [198], it is shown that conductances calculated based on the equilibrium MD approach are consistent with transmission describing diffusive scattering while the NEMD framework consistently describes specular phonon scattering processes as determined for interfaces consisting of atomically perfect LennardJones solids.

Even though we cannot quantitatively prescribe an accurate finite interface resistance, the NEMD-predicted temperature profiles across our crystalline and amorphous films suggest that the resistance across a single crystalline/crystalline interface is greater than that at a single cystalline/amorphous interface. This result is consistent with recent findings of interfacial resistance being higher across a graphene/crystalline-SiC interface compared to a graphene/amorphous-SiC interface, which was mainly attributed to a better overlap of density of states between the graphene and amorphous-SiC compared to crystalline-SiC [200]. Beechem et al. [201] have shown that interfacial resistances predicted under the dif- 
fuse mismatch model approach demonstrate lower resistances for an amorphous/crystalline interface as compared to the predictions for the crystalline counterparts. They attribute the lower resistance at an amorphous/crystalline interface to the lack of abruptness of the system's change from one material to another, which ultimately augments heat flow across the disordered interface. These results allude to the fact that nanoscale imperfections around an interface could result in higher energy transmission across the interface.

For the $\mathrm{Si} / \mathrm{a}-\mathrm{Ge} / \mathrm{Si}$ structures, the values of TBR do not agree with those of the $\mathrm{Si} / \mathrm{c}$ $\mathrm{Ge} / \mathrm{Si}$ for all film thicknesses considered. This can be mainly attributed to the lower thermal conductivities of the amorphous Ge compared to its crystalline counterpart. Increasing the mass of the "Ge" layer by a factor of two also increases the TBR across the thin films (red triangles in Fig. 5.2). In contrast, for the $\mathrm{Ge} / \mathrm{Si} / \mathrm{Ge}$ structures with amorphous and crystalline films, the TBRs for the thinner films (with $L_{\mathrm{f}}<5 \mathrm{~nm}$ ) are comparable in values with each other, regardless of the order and mass of the interfacial region. Although, the band-width in the density of states for amorphous and crystalline Si are very similar to each other (Fig. 5.2 (b)) the heat carrying vibrations in the two phases are considerably different due to the fact that the mean-free-paths of vibrations in the crystal are much longer than those in amorphous $\mathrm{Si}$. This suggests that for thinner Si films (with $L_{\mathrm{f}}<5 \mathrm{~nm}$ ) confined between Ge leads, the order and thus the "mean-free-path" of the vibrations in the film does not significantly influence the TBR as much as the relative frequencies of the film and the leads (i.e., the spectral phonon "mismatch"). Therefore, to evaluate the spectral bandwidth effect on the TBR across Ge/a-Si/Ge structures, we perform additional NEMD simulations by varying the mass of the a-Si, which effectively alters the available frequency modes in the thin films.

The TBRs across Ge/a-Si/Ge with $m_{\text {film }}=14 \mathrm{~g} \mathrm{~mol}^{-1}$ to $112 \mathrm{~g} \mathrm{~mol}^{-1}$ are plotted as a function of mass ratio between the film and the leads in Fig. 5.3 (a). Within uncertainties, the MD predicted TBRs for $28.09 \mathrm{~g} \mathrm{~mol}^{-1}<m_{\text {film }}<84.27 \mathrm{~g} \mathrm{~mol}^{-1}$ are similar. However, for relatively higher or lower masses of the thin film, the TBRs increase considerably. As evident from the MD predicted temperature profiles, part of the reason for the increase in 

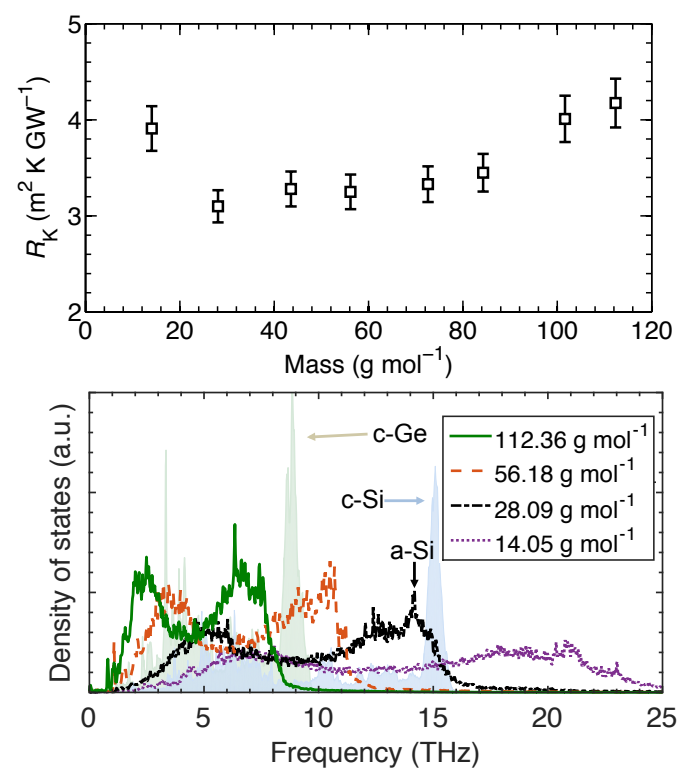

Figure 5.3: (a) TBR of Ge/a-Si/Ge structures with $L_{\mathrm{f}}=3 \mathrm{~nm}$ as a function of mass-mismatch between the leads and the film. (b) Vibrational density of states of crystalline Si and Ge (shaded region), and Si structures with different mass ratios compared to the mass of the Ge lead.

TBRs for the heavier masses is due to the reduction in thermal conductivity with increasing mass $\left(m_{\text {film }}\right)$, while part of it is also due to the mismatch in the overlap of density of states of the film and the leads (calculated from the method described in Ref. [67]) as shown in Fig. 5.3 (b). The cutoff frequencies for the vibrational spectra shown in Fig. 5.3 (b) decrease with increasing mass due to the relation, $\omega \propto 1 / \sqrt{m_{\text {film }}}$. The vibrational spectra for $28.09 \mathrm{~g}$ $\mathrm{mol}^{-1}<m_{\text {film }}<84.27 \mathrm{~g} \mathrm{~mol}^{-1}$ falls within the cutoff frequency of the crystalline Ge leads. Therefore, a significant difference in the TBR across films within this mass range is not observed. However, for the film with relatively lighter mass $\left(m_{\mathrm{film}}=14 \mathrm{~g} \mathrm{~mol}^{-1}\right)$, even though the thermal conductivity increases due to reduced mass, the drastic mismatch in the overlap of density of states with that of the Ge leads (as shown in Fig. 5.3(b)) significantly increases the TBR across the Ge/light-a-Si/Ge structure. This suggests that the resistance across an individual amorphous/crystalline interface can be increased by significantly enhancing the mismatch between the density of the states of the two materials by effectively creating a high mass ratio between the materials. This could be particularly beneficial in de- 
signing SLs with small period thicknesses (and high interface densities) where low thermal conductivities are desired.

\subsection{Thermal transport across amorphous/amorphous su- perlattices}

\subsubsection{Computational details}

For the amorphous-based SLs, the "silicon-like" computational domains were created by starting with a single species of atoms arranged along a diamond cubic lattice with a lattice constant of $5.44 \AA$; two domain lengths were considered to check for finite size effects. Interatomic interactions were specified by the Stillinger Weber (SW) potential parameterized for Si (Ref. [202]), Ge (Ref. [203]) and Si-Ge (Ref. [204]). A time step of 1 fs was used throughout the duration of the simulations and periodic boundary conditions were initially applied in the $x$-, $y$-, and $z$-directions during equilibration and later altered during NEMD. The crystals were heated from $0 \mathrm{~K}$ to above the melt temperature via a velocity scaling routine. They were then allowed to equilibrate by imposing a NVE integration for $1 \times 10^{5}$ time steps followed by NPT integration at zero-pressure and $2500 \mathrm{~K}$ for another $1 \times 10^{5}$ time steps. Next, the crystals were rapidly quenched to near $0 \mathrm{~K}$ by applying a large damping force to all atoms (with a damping coefficient of $0.031 \mathrm{eV} \mathrm{ps}^{-1}$ ). Note, using a quench rate of $10^{12} \mathrm{~K} \mathrm{~s}^{-1}$ produced statistically invariant thermal conductivities for these amorphous SLs. The distributions of atomic coordination numbers and atomic density were calculated to ensure no voids formed during solidification. For the fully amorphous SLs, the atomic masses were varied depending on the position of the atoms in the computational cells in order to create mass-mismatched SLs. 


\subsubsection{Thermal boundary resistance and thermal conductivity of amor- phous Stillinger Weber-based Si/Ge superlattices}

The thermal conductivities of fully amorphous SW Si, Ge, and Si/Ge SLs were calculated using two different domain lengths, $d=125 \AA$ and $250 \AA$. Generally speaking, domain length can have a significant influence on the thermal conductivities predicted by NEMD simulations due to the fact that the fixed ends of the domain serve as phonon scattering sites, thereby shortening mean-free-paths and reducing observed thermal conductivities [129, 205, 206]. However, we do not observe any statistically significant change in the predicted thermal conductivities of amorphous Si or the amorphous SLs (see comparison in Fig. 5.4). While this may seem obvious, it is important to note that recent works in Refs. [207] and [182] have illustrated that a significant portion of the vibrations in amorphous SW-Si are propagating delocalized modes (propagons). However, the period and sample thicknesses for the multilayers studied here are not in a regime where a significant portion of the heat is carried by these propagating vibrational modes. This alludes to the fact that the majority of heat carrying vibrations can be described as diffusons in the SWbased SLs studied in this work (i.e., amorphous SW systems with thicknesses less than $250 \AA \AA$ ). Similarly, size effects have also been reported for crystalline LJ SLs where it was shown that extrapolation methods have to be applied to correctly predict the thermal conductivities [208, 209]. As with the SW-based SLs, the simulations with two domain sizes (160 $\AA$ and $220 \AA$ ) for the amorphous LJ-based multilayers produce statistically invariant thermal conductivities suggesting that no size effects are prevalent for these samples either.

The thermal conductivities of amorphous Si/Ge SLs are plotted as a function of period length, $L$, and interface density, $N$, in Figs. 5.4 and 5.4p, respectively. As shown in the figure, the thermal conductivity of an amorphous Si/Ge SL increases with increasing $L$ and decreases linearly with increasing $N$. These data demonstrate that interfaces contribute a non-negligible thermal resistance. The TBR at an a:Si/a:Ge interface is determined by applying the widely used thermal circuit model, which treats the thermal resistivity of a 

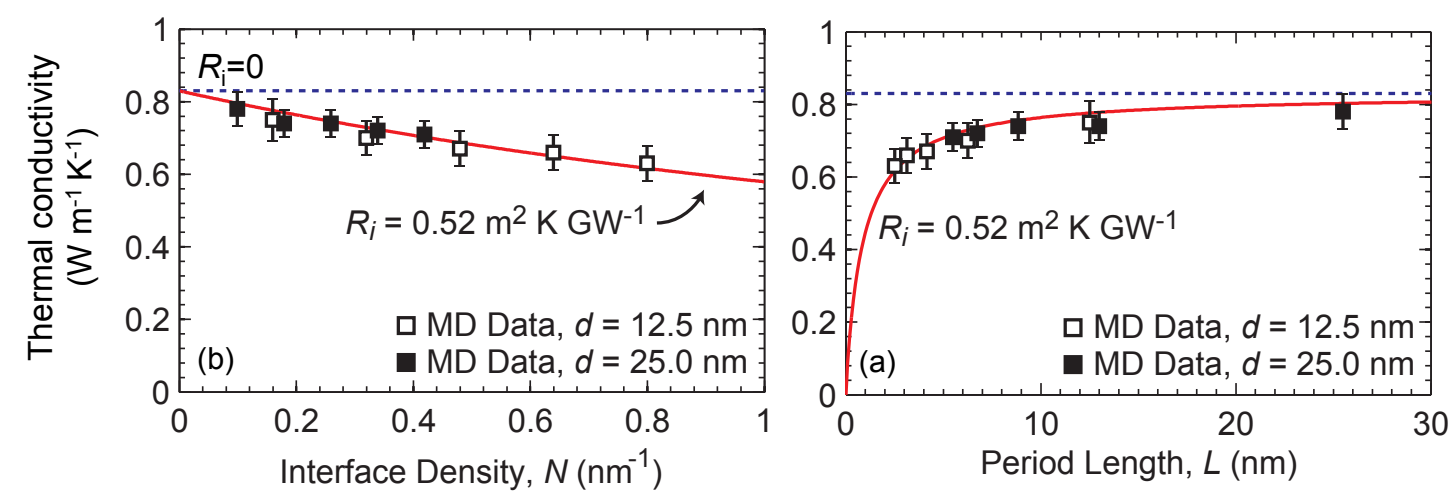

Figure 5.4: Thermal conductivities of amorphous $\mathrm{Si} / \mathrm{Ge}$ superlattices plotted as a function of (a) period length and (b) interface density. Hollow symbols are data from simulations with domains $125 \AA$ long, and solid symbols are data from simulations with domains $250 \AA$ long. The overlap of hollow and solid symbols indicates size effects did not distort the results. Also plotted is the thermal conductivity predicted by Eq. 5.1 when interfaces are ignored (dashed line), as well as with the best-fit value of $R_{\mathrm{i}}=0.52 \mathrm{~m}^{2} \mathrm{KGW}^{-1}$ (solid line). These results are for $\mathrm{Si} / \mathrm{Ge}$ SLs in which the layers are defined by the same interaction parameters in the potential but differ in their mass $\left(m_{\mathrm{A}} / m_{\mathrm{B}}=2.6\right)$.

SL, $\rho$, as a superposition of the thermal resistances of the individual layers and the TBRs at the interfaces. That is,

$$
\rho=\kappa^{-1}=\frac{1}{L}\left[\frac{L}{2 \kappa_{\mathrm{A}}}+\frac{L}{2 \kappa_{\mathrm{B}}}+2 R_{\mathrm{K}}\right]
$$

where $\kappa_{\mathrm{A}}$ and $\kappa_{\mathrm{B}}$ are the thermal conductivities of the constituent components as determined from separate simulations of amorphous Si or Ge. The NEMD-predicted thermal conductivities of amorphous $\mathrm{Si}$ and Ge (with a domain length of $25 \mathrm{~nm}$ and a crosssectional area of $24 \mathrm{~nm}^{2}$ ) are $1.09 \pm 0.14 \mathrm{~W} \mathrm{~m}^{-1} \mathrm{~K}^{-1}$ and $0.67 \pm 0.06 \mathrm{~W} \mathrm{~m}^{-1} \mathrm{~K}^{-1}$, respectively. Note, for the period thicknesses considered in this work, the thermal conductivities of the amorphous materials in each layer in the SW-based SLs are statistically invariant from the thermal conductivities predicted for our "bulk" structures (i.e., $\kappa_{\mathrm{A}}$ and $\kappa_{\mathrm{B}}$ are size independent for these period thicknesses). The NEMD-predicted thermal conductivity for amorphous Si is consistent with the Allen-Feldman theory [179] $(\kappa=1.2 \pm 0.1$ 
$\mathrm{W} \mathrm{m}^{-1} \mathrm{~K}^{-1}$ ) and is also consistent with the thermal conductivity predicted via normal mode decomposition analysis of amorphous $\mathrm{Si}$ that only considers the contribution from non-propagating modes as discussed in Ref. [182]. However, as mentioned earlier, size effects can drastically increase the thermal conductivity of amorphous Si for large simulation domains where the significant amount of heat is carried by propagons [182, 210]. In Ref. [210], NEMD results on domain lengths smaller than $\sim 33 \mathrm{~nm}$ did not show a noticeable size dependence in the predicted thermal conductivity as non-propagating modes do not contribute significantly to thermal transport on this length scale. This is consistent with the results of our NEMD simulations where any size effects in thermal conductivities for the domain lengths and layer thicknesses used for our SW-based SLs are not observed, further validating the use of thickness independent thermal conductivities as input parameters in Eq. 5.1 for these SLs.

Using Eq. 5.1, the thermal conductivity data, and a least squares fitting routine, it is found that the TBR at an a:Si/a:Ge interface is $0.52 \mathrm{~m}^{2} \mathrm{~K} \mathrm{GW}^{-1}$. The thermal conductivities predicted from the least squares fitting routine are within $4 \%$ of the values determined by the NEMD simulations, demonstrating that the model fits the MD data very well. The best-fit value of $R_{\mathrm{K}}$ is consistent with that calculated from separate NEMD simulations of isolated interfaces between amorphous $\mathrm{Si}$ and $\mathrm{Ge}$ (corresponding to temperature profiles). More specifically, a temperature drop of $3.6 \mathrm{~K}$ is expected from the resistance predicted by Eq. 5.1 at the amorphous Si/heavy-Si interface. From the temperature profile for the particular computational domain at the isolated interface, a temperature drop of $\sim 4 \mathrm{~K}$ is observed, which is in excellent agreement with the prediction from the thermal circuit model, suggesting that the resistance predicted by Eq. 5.1 can be used to describe the internal resistances in these SW-based amorphous SLs.

It is interesting to note that the resistance at an a:Si/a:Ge interface is $\sim 6$ times lower than at the corresponding isolated interface between crystalline Si and Ge (as determined via additional simulations on an isolated crystalline $\mathrm{Si} / \mathrm{Ge}$ interface and further verified by a previous work that studied the resistance at isolated crystalline Si/Ge interfaces [189]). 
More specifically, from the additional simulations on an isolated crystalline $\mathrm{Si} / \mathrm{Ge}$ interface, the resistance is $2.81 \mathrm{~m}^{2} \mathrm{~K} \mathrm{GW}^{-1}$ at this interface; note that for this crystalline $\mathrm{Si} / \mathrm{Ge}$ simulation, the species differ only in mass and simulations are conducted using a similar domain size as studied for the amorphous structures at $500 \mathrm{~K}$. The result on the mass-mismatched crystalline interface is within 5\% of the MD prediction from Landry and McGaughey $\left(R_{\mathrm{K}}=2.93 \mathrm{~m}^{2} \mathrm{~K} \mathrm{GW}^{-1}\right)$ for a crystalline Si/Ge interface [189]. The small discrepancy between the predicted resistances might be due to the fact that our simulations do not consider the strain associated with the lattice mismatch between $\mathrm{Si}$ and $\mathrm{Ge}$, whereas, the MD simulations in Ref. [189] consider the lattice mismatch between the species and also take into account the different interaction parameters between the species. Moreover, the fact that the domain size for the crystalline $\mathrm{Si} / \mathrm{Ge}$ structure simulated in this work is well below the mean free path of heat carrying phonons in these structures, size effects can significantly influence the predicted resistances across crystalline $\mathrm{Si} / \mathrm{Ge}$ interfaces as shown by Landry and McGaughey's work [189]. For a comprehensive study of TBR at crystalline $\mathrm{Si} / \mathrm{Ge}$ and $\mathrm{Si} /$ heavy-Si interfaces, the reader is referred to Ref. [189] where the authors compare their MD and lattice dynamics results to theoretical calculations.

The difference in the resistances between amorphous and crystalline $\mathrm{Si} / \mathrm{Ge}$ interfaces is despite the fact that the vibrational mismatch between amorphous $\mathrm{Si}$ and $\mathrm{Ge}$ is very similar to that between crystalline $\mathrm{Si}$ and Ge (see Fig. 5.3b). While the vibrational bandwidths of these two materials are similar regardless of amorphicity or crystallinity, the vibrations that predominately contribute to thermal transport in our amorphous $\mathrm{Si}$ and Ge layers in the SLs are non-propagating modes. That is, the heat carrying vibrations in the amorphous $\mathrm{Si} / \mathrm{Ge} \mathrm{SLs}$ studied in this work are not spatially extended as in the case of crystalline $\mathrm{Si} / \mathrm{Ge}$ systems (this conclusion can be drawn due to the absence of size effects in the context of the former and the prevalence of size effects in the context of the latter). This is supported by the simulations on LJ-based samples as well, where size effects are not observed as mentioned above.

It is also interesting to note that resistances at the interfaces within the amorphous 
SW SLs do not appear to be a function of interface density. On the contrary, TBR has been shown to decrease with increasing interface density in crystalline SLs [4, 211]. This behavior has been ascribed to a transition from diffusive to ballistic phonon transport, i.e., a shortening of phonon mean-free-paths [4]. Taking these two observations together, it follows that interfacial thermal transport is mediated by delocalized and non-propagating modes (or diffusons) in these amorphous SLs. In other words, recent works have shown that the thermal boundary conductance (TBC; $R_{\mathrm{K}}^{-1}$ ) across interfaces in SLs can increase when the SL period is less than the phonon mean free path [4] suggesting that long wavelength phonons contribute to TBC differently than short wavelength phonons; this trend is not observed for the simulations performed in this work for the $\mathrm{Si} / \mathrm{Ge}$ or $\mathrm{Si} / \mathrm{heavy-Si} \mathrm{SLs}$, which indicates that TBC in these amorphous SLs is mediated by diffusons.

\subsubsection{Experimental investigation on the thermal properties of amor- phous superlattices}

To experimentally investigate the thermal properties of amorphous SLs and to validate the computational results presented in the previous section, alternating stacks of SiOC:H and $\mathrm{SiC}: \mathrm{H}$ layers with varying period thicknesses are measured via TDTR. The SL samples were deposited on Si substrates via plasma-enhanced chemical vapor deposition (PECVD) carried out at the Intel corporation (collaboration with Dr. Sean King). Additionally, a thickness series of homogeneous $\mathrm{SiOC}: \mathrm{H}$ and $\mathrm{SiC}: \mathrm{H}$ thin films were also grown on $\mathrm{Si}$ substrates via PECVD. The film and period thicknesses were determined via XRR and XSEM measurements (which were also carried out at Intel). Note, the XRR and XSEM data confirm the periodic arrangement of $\mathrm{SiOC}: \mathrm{H} / \mathrm{SiC}: \mathrm{H}$ layers, with period thicknesses ranging from $\sim 3.4$ to $26.4 \mathrm{~nm}$.

To accurately determine the thermal conductivities of the SiOC:H/SiC:H SLs, the heat capacities of the constituent layers in the SL must be determined first. Along with the thermal conductivities of the SLs, to accurately determine the intrinsic resistance at a single 
SiOC:H/SiC:H, the thermal conductivities of the individual layers are required (as input parameters to Eq. 5.1). For this purpose, the thermal conductivities and heat capacities of the thickness series of homogeneous SiOC:H and $\mathrm{SiC}: \mathrm{H}$ films are determined with a similar approach as implemented for the hybrid SL films in the previous chapter. In this context, different pump-modulation frequencies are utilized to simultaneously measure the thermal conductivities and heat capacities of $\mathrm{SiC}: \mathrm{H}$ and SiOC:H thin films. Figure 5.5 shows the sensitivities of the ratio to the thermo-physical parameters in the thermal model for a $\sim 88$ $\mathrm{nm}$ thick $\mathrm{SiC}: \mathrm{H}$ film at 2 and $10 \mathrm{MHz}$ pump-modulation frequencies. The most sensitive parameters in the model are the heat capacity and the thermal conductivity of the thin film. However, for a particular frequency, a range of heat capacities can produce the best-fit to the experimental data as shown in the contour plot of the residual error. The common heat capacity and thermal conductivity value that produces the best-fit to the data for the two frequencies is taken as the measured values for the homogeneous samples. This approach results in a thermal conductivity of $1.48 \pm 0.12 \mathrm{~W} \mathrm{~m}^{-1} \mathrm{~K}^{-1}$ and $0.75 \pm 0.06 \mathrm{~W} \mathrm{~m}^{-1} \mathrm{~K}^{-1}$ and measured values for the volumetric heat capacities of $1.9 \pm 0.3 \mathrm{~J} \mathrm{~cm}^{-3} \mathrm{~K}^{-1}$ and $1.3 \pm 0.2 \mathrm{~J}$ $\mathrm{cm}^{-3} \mathrm{~K}^{-1}$ for the $\mathrm{SiC}: \mathrm{H}$ and SiOC:H, respectively.

Figure 5.6 plots the measured thermal conductivity as a function of film thickness for
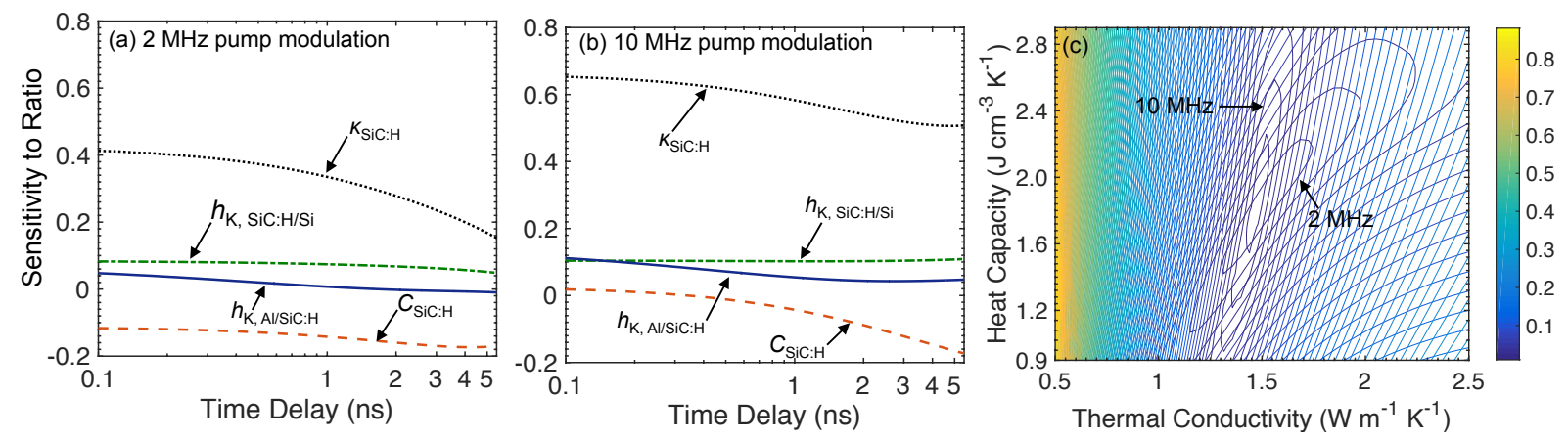

Figure 5.5: Sensitivities of ratio $\left(-V_{\text {in }} / V_{\text {out }}\right)$ to the thermophysical properties of the $88 \mathrm{~nm}$ thick SiC:H sample as a function of pump-probe time delay at (a) $2 \mathrm{MHz}$ pump modulation frequency and (b) $10 \mathrm{MHz}$ pump modulation frequency. (c) Sensitivity contour plot showing the interrelationship between thermal conductivity and heat capacity of the $88 \mathrm{~nm}$ $\mathrm{SiC}: \mathrm{H}$ sample at $2 \mathrm{MHz}$ and $10 \mathrm{MHz}$ pump modulation frequencies. 


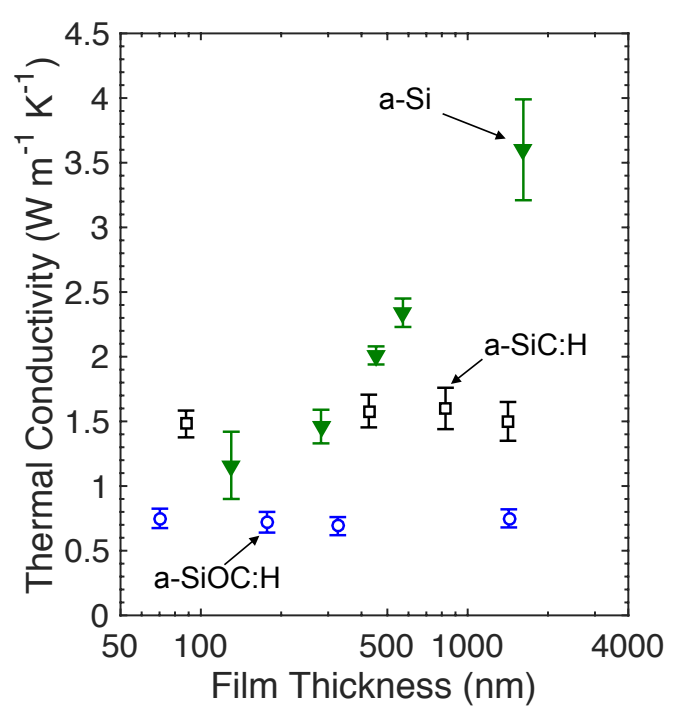

Figure 5.6: Thermal conductivity of amorphous $\mathrm{SiC}: \mathrm{H}$ and $\mathrm{SiOC}: \mathrm{H}$ films as a function of film thickness. The lack of size effects for both amorphous structures suggest that thermal transport is mostly mediated by diffusons. Also included are the measured thermal conductivities of amorphous Si from Ref. [212], which shows considerable size effects due to the contribution from propagating modes to the thermal conductivity.

the homogeneous thin films. The lack of film thickness dependence on the thermal conductivity of the amorphous $\mathrm{SiOC}: \mathrm{H}$ and $\mathrm{SiC}: \mathrm{H}$ structures suggests that heat conduction in these films is mostly driven by vibrations that are classified as diffusons. This is in contrast to our recent experimental results (from Ref. [212], which is also included in Fig. 5.6) demonstrating size effects on the thermal conductivity of amorphous Si thin films, where a significant portion of heat flow is due to propagons (that represent delocalized propagating modes). The lack of size effects in the thermal conductivity of a-SiOC:H can be attributed to the Si-O-Si network structure (confirmed from FTIR measurements on a similar a-SiOC:H film as reported in Ref. [186]) that is similar to the structure found $\mathrm{in}^{\mathrm{SiO}_{2}}$; the lack of size effects in $\mathrm{SiO}_{2}$ is significantly because of the weak bonding that exists between the $\mathrm{SiO}_{4}$ tetrahedra whereas the thickness dependent thermal conductivity in a-Si is because of the strongly bonded tetrahedra[182]. For the a-SiC:H, the network structure mostly shows Si-C stretching modes similar to a-SiC systems [186, 213]; the lack of size effects in the a$\mathrm{SiC}: \mathrm{H}$ is consistent with size independent thermal conductivities measured for amorphous 
$\mathrm{SiC}$ in Ref. [214]. These findings along with the measurement of heat capacities for the amorphous SiOC:H and $\mathrm{SiC}: \mathrm{H}$ films will be utilized to derive a mean TBR across a single $\mathrm{SiOC}: \mathrm{H} / \mathrm{SiC}: \mathrm{H}$ interface by measuring the thermal properties of SiOC:H/SiC:H SLs with varying period thicknesses as detailed in the discussions below.

\subsubsection{Thermal boundary resistance and thermal conductivity of amor- phous SiC:H/SiOC:H superlattices}
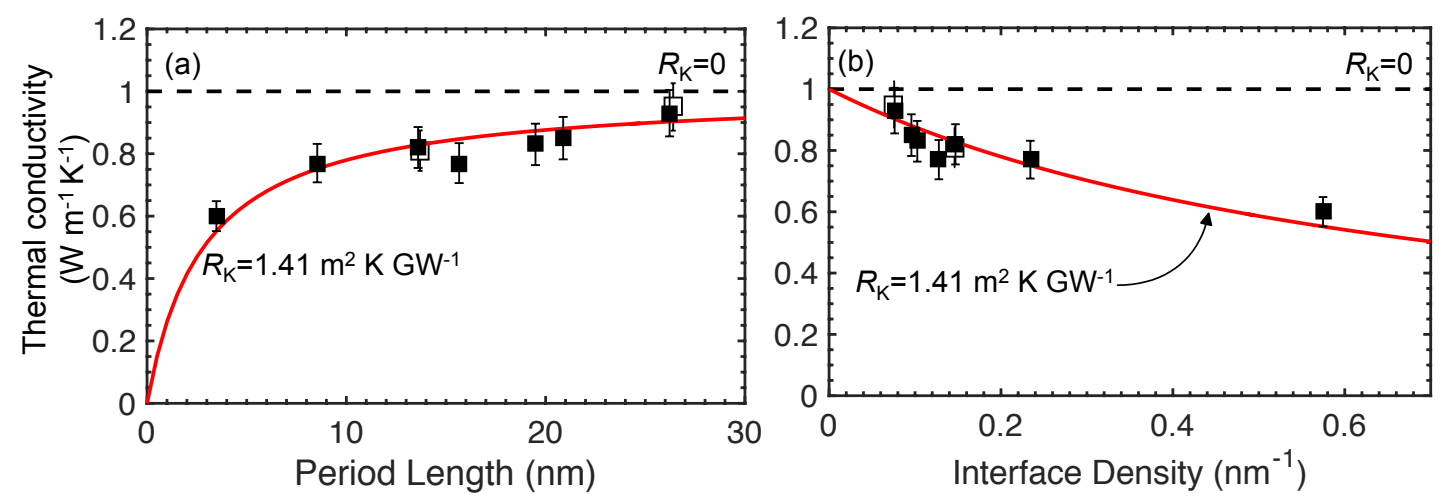

Figure 5.7: Thermal conductivities of amorphous $\mathrm{Si} / \mathrm{Ge}$ superlattices plotted as a function of (a) period length and (b) interface density. The hollow symbols are for structures with similar period thicknesses and larger total film thicknesses compared to the ones with the solid symbols.

The thermal conductivity of amorphous $\mathrm{SiC}: \mathrm{H} / \mathrm{SiOC}: \mathrm{H}$ superlattices is shown as a function of period length and interface density in Fig. 5.7 a and Fig. 5.7b, respectively. Similar to the computational results on the $\mathrm{SW} \mathrm{Si/Ge} \mathrm{SLs,} \mathrm{the} \mathrm{experimentally} \mathrm{determined} \mathrm{thermal}$ conductivity for $\mathrm{SiC}: \mathrm{H} / \mathrm{SiOC}: \mathrm{H}$ SLs monotonically decreases with decreasing period thickness and increasing interface density. This suggests that the interfaces in the amorphous SLs contribute non-negligibly to thermal resistance across the thin films. To determine this resistance across a single $\mathrm{SiC}: \mathrm{H} / \mathrm{SiOC}: \mathrm{H}$ interface, Eq. 5.1 is fit to the experimental data with $R_{\mathrm{K}}$ as the fitting parameter. This approach predicts $R_{\mathrm{K}}=1.41 \mathrm{~m}^{2} \mathrm{~K} \mathrm{GW}^{-1}$ $\left(h_{\mathrm{K}}=710 \mathrm{MW} \mathrm{m}{ }^{-2} \mathrm{~K}^{-1}\right.$ ), which is considerably lower than the typical values reported for 
crystalline/crystalline interfaces (which range from 5 to $50 \mathrm{~m}^{2} \mathrm{~K} \mathrm{GW}^{-1}[13]$ ).

The low TBR (high TBC) across amorphous interfaces measured for the SiC:H/SiOC:H SLs is in line with the low resistances predicted for Si/Ge SLs via MD simulations. These results are also consistent with $R_{\mathrm{K}}=1.5 \mathrm{~m}^{2} \mathrm{~K} \mathrm{GW}^{-1}$ measured across $\mathrm{SiO}_{2} / \mathrm{Al}_{2} \mathrm{O}_{3}$ interfaces in Ref. [215]. As mentioned in the discussion for the MD-predicted TBR on amorphous $\mathrm{Si} / \mathrm{Ge} \mathrm{SLs}$, the spectrum of frequencies in the density of states for the amorphous material remains similar to that of the cystalline counterpart, however the heat carrying vibrations are very different between the amorphous and crystalline phases. For an amorphous/amorphous interface, the better overlap between the heat carrying vibrations on either side of the interface (even though the acoustic mismatch between the layers is large) results in the lower TBR as compared to interfaces that are comprised of highly dissimilar crystalline materials. These findings will be utilized in the following section to design SLs with tunable thermal properties.

\subsection{Thermal transport across amorphous/crystalline su- perlattices}

\subsubsection{Computational details}

For our amorphous/crystalline SLs, the crystalline layers of the SL are prepared by deleting atoms in the amorphous domain according to the position of the atoms and then substituting the deleted atoms with atoms arranged along a diamond cubic lattice with an average value of the lattice constants of Si and Ge. A similar method has also been implemented in Refs. [216, 217] to produce Si-based amorphous/crystalline structures. The species of atoms between the amorphous and crystalline layers were separated depending on the position of the atoms in the computational cell and the final structures are relaxed with the same procedure as for the fully amorphous SLs. The radial distribution function and atomic coordination numbers were calculated for each amorphous and crystalline layer 


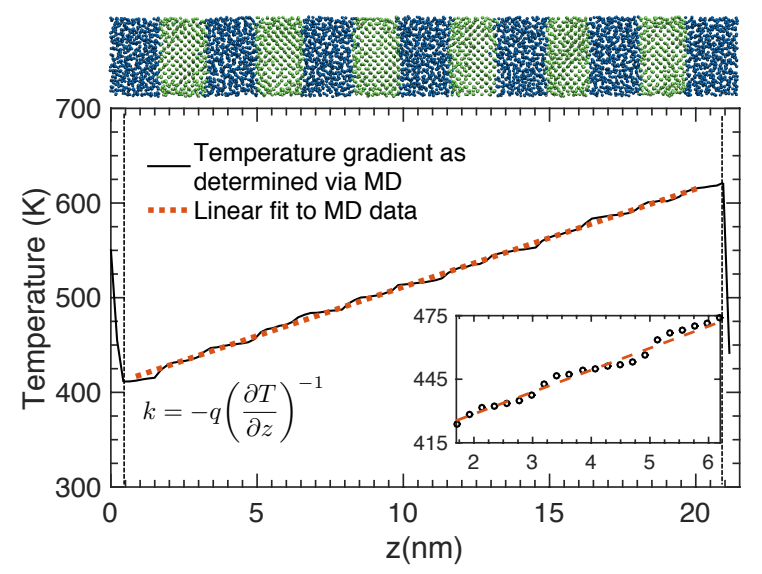

Figure 5.8: (Top panel) Schematic of a $27 \times 27 \times 212 \AA^{3}$ simulation cell with $N=0.57 \mathrm{~nm}^{-1}$ for an amorphous/crystalline superlattice. (Bottom panel) Temperature gradient induced due to the applied flux across the computational domain. (Inset) Local temperature profiles for the amorphous/crystalline layers are similar because of high interface densities.

in the SL structures to ensure no voids are formed during and after energy minimization. It is well known that for amorphous solids, the thermal conductivity is highly dependent on the density of the solid [121, 150, 184]. Therefore, we determine the densities of each a-Si and c-Ge layer (based on the local volume and the number of atoms present in the respective layers in the relaxed SL structures). We confirm that the calculated densities match with their bulk counterparts for all of our SL structures with different interface densities.

The cross section area for the SLs is set to five by five unit cells with periodic boundary conditions in the $x$ - and $y$-directions. The top panel of Fig 5.8 shows an example of the computational domain created for the cystalline/amorphous superlattices and the bottom panel shows the temperature gradient induced by the steady-state heat flux applied across the computational domain (with a similar procedure as explained above for the confined thin films); note, similar procedure was implemented for the fully amorphous SLs as well. From the temperature gradient, the thermal conductivity is calculated by invoking the Fourier law as shown in Fig. 5.8. The local temperature profiles (as shown in the inset of Fig. 5.8) for the amorphous and crystalline layers are similar because of the high interface densities in these structures, however, we note that the radial distribution functions calculated for the crystalline layers show proof of crystallinity in those layers. 


\subsubsection{Thermal conductivity of amorphous/crystalline Si/Ge superlat- tices}

The thermal conductivities of amorphous/crystalline Si/Ge SLs were predicted for two different domain lengths, $d=21 \mathrm{~nm}$ and $43 \mathrm{~nm}$. As mentioned above, domain lengths have been shown to significantly influence the thermal conductivities. This is exemplified by the thermal conductivities plotted as a function of interface density in Fig. 5.9 for SWbased crystalline/crystalline Si/Ge SLs that are taken from Ref. [218] (solid triangle and diamond symbols). As is clear, the two domain lengths produce completely different thermal conductivities for the c-Si/c-Ge SLs, suggesting that size effects can greatly influence the thermal conductivities (by as much as four-fold) in these superlattice structures. In contrast to these cystalline/crystalline SLs, no statistically significant change in the predicted thermal conductivities is observed for the amorphous/crystalline SLs as is evident from the overlap of the predicted thermal conductivities for the two domain lengths in Fig. 5.9 (hollow and solid squares).

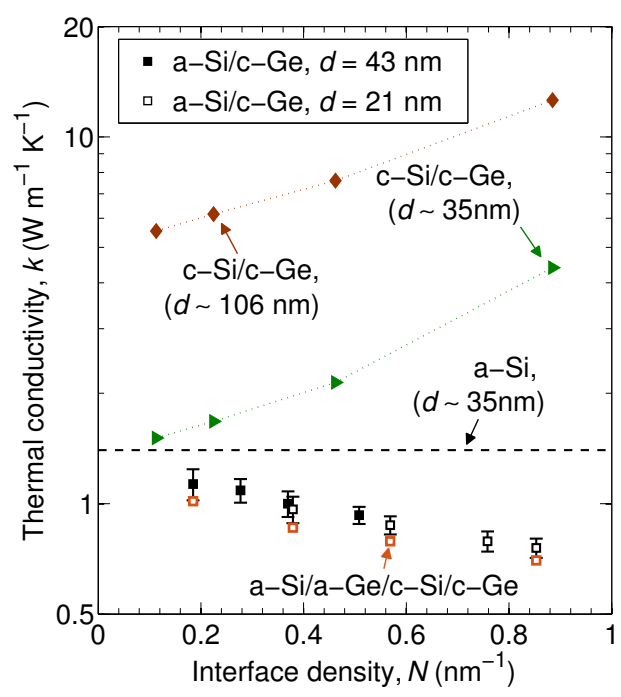

Figure 5.9: Thermal conductivities of $\mathrm{Si} / \mathrm{Ge} \mathrm{SLs}$ as a function of interface density $N$. Solid square symbols are MD predictions on a-Si/c-Ge SLs with domain lengths of $21 \mathrm{~nm}$ while hollow square symbols are predictions for a-Si/c-Ge SLs with domain length of $43 \mathrm{~nm}$. For comparison, MD predictions taken from Ref. [218] on crystalline Si/Ge superlattices with two domain lengths are also plotted. 
The MD-predicted thermal conductivities for the amorphous/crystalline SLs decrease linearly with increasing $N$, implying that interfaces contribute non negligibly to thermal resistance in these structures, similar to the fully amorphous SLs. The trend observed in Fig. 5.9 also suggests that vibrations are mostly particle-like in nature and incoherent scattering at the internal boundaries of the SLs leads to a monotonic reduction in thermal conductivity with increasing $N$. In contrast, for the crystalline/crystalline SLs, the thermal conductivity increases with increasing $N$, which can be attributed to the wave nature of phonon transport in structures where the phonons do not scatter at the internal boundaries of the SLs or to ballistic transport across relatively thicker periods [5, 155, 219]; note, this coherence effect in the calculated thermal conductivity of SW-Si/Ge crystalline SLs with "perfect" interfaces has been previously observed via MD simulations [211].

The thermal conductivities of the amorphous/crystalline SLs are below the thermal conductivity of amorphous $\mathrm{Si}$ as predicted by additional MD simulations (dotted line in Fig. 5.9. Therefore, by creating interfaces, the thermal conductivity can be lowered even below that of the amorphous material, which is often thought to be the minimum limit to thermal conductivity in materials. Compared to the amorphous/amorphous SLs, the thermal conductivities of the amorphous/crystalline structures are slightly higher. As discussed above, the resistance as compared to their crystalline counterparts, across a single a-Si/a-Ge interface can be six times lower. This implies that creating SL structures with two amorphous layers and two crystalline layers forming a period in the SL would lead to lower thermal conductivities because of the higher resistances across c-Si/c-Ge interfaces relative to a-Si/a-Ge interfaces. To validate this hypothesis, additional NEMD simulations on SLs structures with a-Si/a-Ge/c-Si/c-Ge are performed and it is found that the thermal conductivities of these structures are systematically $\sim 10 \%$ lower than the a-Si/c-Ge structures with the same layer thicknesses (red squares in Fig. 5.9). Thus, strategic placement of layers and control over the thicknesses of the individual layers in these SLs can produce structures with thermal conductivities that are below the minimum limit of their amorphous counterparts. 


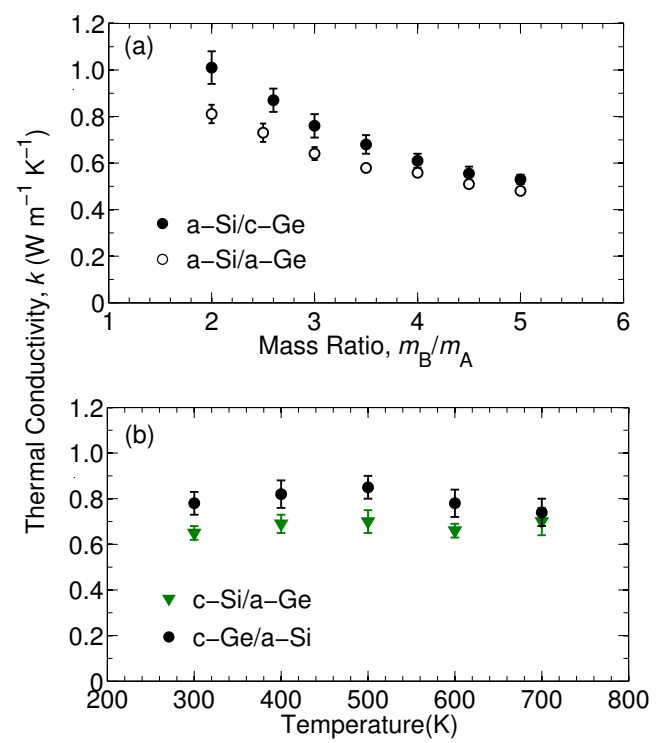

Figure 5.10: (a) Thermal conductivity of a-Si/c-Ge SLs with $N=0.57 \mathrm{~nm}^{-1}$ plotted as a function of mass-mismatch between the layers. (b) Thermal conductivity of amorphous/crystalline $\mathrm{Si} / \mathrm{Ge} \mathrm{SLs}$ described with the Tersoff potential (layers differentiated by mass and bond).

The thermal conductivities of the amorphous/crystalline SLs can be further lowered by increasing the mass-mismatch of the layers in the SL. Figure 5.10 (a) shows the thermal conductivities of a-Si/c-Ge with $d=21 \mathrm{~nm}, N=0.57 \mathrm{~nm}^{-1}$ and with the mass of the Ge layer ranging from $56 \mathrm{~g} \mathrm{~mol}^{-1}$ to $145 \mathrm{~g} \mathrm{~mol}^{-1}$ (solid circles). As is evident in the plot, the thermal conductivities of these SLs decrease with increasing mass-mismatch. The reduction in thermal conductivity is because of the combined effect of increase in the TBR and decrease in the thermal conductivity of the layers from the increase in mass, as mentioned above for the confined thin films. For comparison, the MD predicted thermal conductivities of mass-mismatched fully amorphous SLs are also presented. The thermal conductivities for the two different SLs converge as the mismatch between the masses in the layers increases. This demonstrates the ability of vibrational mismatch and resulting TBR at SL interfaces to dominate the thermal transport mechanisms, regardless of the degree of crystallinity of the materials.

The thermal conductivity of the a-Si/c-Ge with $N=0.57 \mathrm{~nm}^{-1}$ is plotted as a func- 
tion of temperature in Fig. 5.10 (b) (solid circles). Along with c-Ge/a-Si systems, c-Si/a-Ge SLs have slightly lower thermal conductivities (as shown in Fig. 5.10(b)), which is mainly because of the lower thermal conductivity of a-Ge compared to a-Si. The thermal conductivities do not show a temperature dependency, contrary to purely crystalline SLs [4, 211] but consistent with results for the fully amorphous SLs. In Ref. [216] similar results are obtained for a-Si/mass-heavy c-Si SLs where they show a very slight increase in thermal conductivity with temperature.

\subsection{Summary}

Molecular dynamics simulations were implemented to investigate the thermal boundary resistance across amorphous and crystalline confined films of $\mathrm{Si} / \mathrm{Ge} / \mathrm{Si}$ and $\mathrm{Ge} / \mathrm{Si} / \mathrm{Ge}$ structures with film thicknesses ranging from $2 \mathrm{~nm}$ to $25 \mathrm{~nm}$. For crystalline thin films, depending on the film and the leads, the combined ballistic and diffusive behavior of phonon transport can influence the thermal boundary resistance. Conversely, for amorphous films, the resistance increases monotonically regardless of the leads or the amorphous material comprising the thin film. From the temperature profiles obtained from the NEMD simulations, the predicted resistances across single amorphous/crystalline interfaces are lower than that across their crystalline counterparts. This suggests that diffusive scattering at amorphous/crystalline interfaces can lead to higher energy transmissions as compared to crystalline/crystalline interfaces for these systems. Finally, for structures with film thicknesses $\lesssim 3 \mathrm{~nm}$, the thermal boundary resistance across the interfacial layer is highly dependent on the overlap of density of states between the leads and the confined films.

The fact that high resistances that arise due to high mass-mismatch between the $\lesssim 3$ $\mathrm{nm}$ confined films and the leads and the fact that crystalline/crystalline interfaces demonstrate higher resistances compared to crystalline/amorphous interfaces is used to design SL structures with very low thermal conductivities. In this regard, NEMD simulations are performed to predict the thermal conductivities of amorphous/crystalline and amor- 
phous/amorphous $\mathrm{Si} / \mathrm{Ge} \mathrm{SLs}$ with varying interface densities and mass ratios between the layers. In contrast to crystalline/crystalline SLs, the thermal conductivities of the amorphousbased SLs do not demonstrate size effects for interface densities above $0.1 \mathrm{~nm}^{-1}$. This suggests that for these interface densities, the wave-nature of phonon transport observed for crystalline $\mathrm{Si} / \mathrm{Ge} \mathrm{SLs}$ is absent in the amorphous-based SLs and delocalized non-propagating modes conduct heat in these structures. The thermal conductivities decrease monotonically with increasing interface densities, which further supports the claim that incoherent scattering of vibrations limits thermal transport across these SLs. These results are corroborated with experimental results on amorphous $\mathrm{SiOC}: \mathrm{H} / \mathrm{SiC}: \mathrm{H}$ superlattices, which demonstrate ultralow thermal conductivities. For an individual SiOC:H/SiC:H interface, a value of 1.41 $\mathrm{m}^{2} \mathrm{~K} \mathrm{GW}^{-1}$ is derived from the thermal conductivity measurements of varying period thickness SLs. This value of interfacial resistance is much lower than the resistances that are usually measured for interfaces comprising crystalline materials.

By designing the period of the SLs to consist of two crystalline layers followed by two amorphous layers, $\mathrm{a} \sim 10 \%$ reduction in the thermal conductivity is demonstrated via MD simulations. This is mainly attributed to the higher resistance at the crystalline/crystalline interface as compared to amorphous/crystalline or amorphous/amorphous interfaces. Furthermore, at high mass ratios between the layers in the amorphous/crystalline SLs, the thermal conductivities of amorphous/crystalline SLs are shown to be comparable to those of amorphous/amorphous SLs despite the cyrstallinity in one of the constituent layers. 


\section{Chapter 6}

\section{Conclusions and future work}

\subsection{Summary of major results}

The field of nanoscale heat transfer is critical to current as well as emerging technologies that rely on accurate and comprehensive description of the fundamental energy carriers. The ongoing miniaturization and the continual increase in the density of interfaces in microelectronic devices has led to size effects that cannot be explained by the conventional Fourier theory and requires approaches based on the Boltzmann transport equation and atomic-level simulations. Even though theoretical tools can provide detailed descriptions of transport, the proper choices of assumptions and computational setup require valid empirical results. In this regard, the work presented in the thesis has focused on resolving the voids in the range of data available for thermal boundary conductance at solid/solid interfaces. Theoretical developments and computational analysis presented in this thesis have also contributed to an important step towards a comprehensive understanding of the microscopic dynamics of energy carriers and how these carriers are affected at interfaces.

The thermophysical basis of energy transport across interfaces is discussed in Chapter 2 and the often used theories to understand interfacial transport such as the diffuse mismatch model and acoustic mismatch model are introduced. In this chapter, a theory of electronphonon interactions at metal/nonmetal interfaces, and the resulting thermal boundary con- 
ductance, in the case when the electrons in the metal are defined by a different temperature than the metal and substrate lattices, is developed and later used in Chapter 3 to help explain some of the experimental observations. Phonon-driven thermal boundary conductance is also discussed in the chapter through implementation of molecular dynamics simulations on toy models based on the Lennard-Jones potential. Through these simulations and the implementation of a modal analysis technique, the influence of weak bonding on interfacial conductance is probed. It is shown that weak bonding at interfaces causes the heat carrying phonons to shift towards lower frequencies, consequently resulting in lower thermal boundary conductances. The chapter also introduces the time domain thermoreflectance technique and emphasizes the critical advancements made in the analysis procedure from the work presented in the thesis.

Chapter 3 attempts to resolve the debate over the direct coupling of metal-electrons to nonmetal-phonons by systematically studying varying thicknesses of homogeneous $\mathrm{Au}$ films and thin bilayer films of $\mathrm{Au} / \mathrm{Ti}$ and $\mathrm{Au} / \mathrm{Pt}$ systems deposited on various dielectric substrates with varying thermal effusivities. One of the major results from the chapter is that electron-metal/phonon non-metal energy transfer does not influence the thermal boundary conductance across metal/nonmetal interfaces when the electrons have fully thermalized with lattice vibrations and only during highly nonequilibrium conditions between the electrons and phonons $\left(T_{\mathrm{e}} \gg T_{\mathrm{p}}\right)$ does eletron-phonon scattering at an interface contribute to thermal boundary conductance.

In Chapter 4, thermal transport in hybrid multilayers based on $\mathrm{ZnO}$ and $\mathrm{TiO}_{2}$ is studied via time domain thermoreflectance. One of the major contributions from the work is the experimental demonstration of "near-prefect" transmission of phonon energy (which is $80 \%$ of maximal conductance across the inorganic $\mathrm{ZnO}$ layer) across an organic monolayer of hydroquinone. The work also marks one of the first observations of molecular vibrons coupling with phonons in the inorganic solids, providing unique opportunities for user defined thermal transport in hybrid multilayers. Along these lines, it is shown that the thermal conductivity of $\mathrm{ZnO}$-based multilayers can be tuned in the range of two orders of 
magnitude, a result that is significant to applications such as thermoelectric devices and thermal barrier coatings.

Chapter 5 examines the effect of disorder on thermal transport across confined thin films and superlattices. A combination of molecular dynamics simulations and time domain thermoreflectance technique are used to achieve these goals. The major contribution from this work is the conclusive evidence of amorphous/crystalline and amorphous/amorphous interfaces posing lower resistances to thermal transport compared to their crystalline counterparts, however, with high interfacial densities, these interfaces are shown to nonnegligibly affect thermal transport in superlattice systems. These observations lead to the design of amorphous-based superlattice structures with ultralow thermal conductivities.

Overall, breaking the limits of thermal transport and questioning conventional wisdom through systematic experimental and well guided theoretical studies has been the driving force behind this thesis. The "big picture" summary of this thesis is that time domain thermoreflectance and molecular dynamics simulations serve as robust tools to further the field of nanoscale heat transfer. It is the hope of the author that the studies presented in this thesis prove to be motivating building blocks for new research in this burgeoning field.

\subsection{Future work}

Although many areas in the field of heat transfer deserve in-depth attention, in the context of the work presented here, several topics of future interest are outlined below.

- Understanding electron dynamics in metals has largely been limited to nobel metals due to their relatively less complicated band structure. Futhermore, studies including the work presented in this thesis have been conducted for electron temperatures $\leq 3,500 \mathrm{~K}$ in Au due to the lack of analytical models that can accurately describe the physics of $d$-band excitation and the concomitant relaxation processes. Experimental studies with high laser energies targeting subcondution band excitations and their implications on electron-boundary scattering will prove to be a major step forward 
in understanding high temperature electron dynamics.

- The in-plane thermal conductivity measurements on the hybrid superlattice films have tremendous implications for thermoelectric devices. Beam-offset time domain thermoreflectance can be implemented to measure the in-plane properties of these material systems. Along these lines, cross-plane electrical resistivity measurements on these films will allow for the accurate prediction of the Seebeck coefficient for these superlattice films.

- To separate the contributions from the scattering at a single $\mathrm{ZnO} / \mathrm{HQ}$ interface and the scattering in the monolayer of HQ, a more in-depth theoretical study utilizing first principles calculations should be conducted. Modeling this heat transfer process across hybrid superlattices will ultimately advance the knowledge of the coupling between phonons in the inorganic material and vibrons in the molecular constituent.

- Most of the atomistic simulations conducted in this work have relied on simplistic potentials such as the Lennard-Jones potential and Stillinger-Weber potential. More complicated and material specific potentials should be developed in order to describe new material systems, which combined with recently formulated modal analysis techniques should be able to shed more light on the spectral nature of vibrational energy in complex material systems such as the ones studied in this thesis.

- The ability to perform laser wavelength spectroscopy on individual vibrational modes will also be a major advance in the field. For example, individual modes in the organic hydroquinone monolayers in the hybrid superlattices could be excited by tuning the laser wavelength to target those particular modes. The consequent energy coupling from these modes to the inorganic layers could be studied through the conventional pump-probe technique. This is also applicable to electrons, where the probe energy can be varied so as to understand the electronic relaxation mechanisms at various energy levels. 
- Finally, the design of amorphous/crystalline superlattices presented in Chapter 5 should be envisioned experimentally and the thermal properties should be studied via time domain thermoreflectance to validate the computational findings. 


\section{Bibliography}

[1] A. Einstein. "Elementare Betrachtungen ber die thermische Molekularbewegung in festen Krpern". In: Annalen der Physik 340.9 (1911), p. 679.

[2] P. Debye. "Zur Theorie der spezifischen Wärmen" In: Annalen der Physik 344.14 (1912), pp. $789-839$.

[3] W. S. Capinski, H. J. Maris, T. Ruf, M. Cardona, K. Ploog, and D. S. Katzer. "Thermal-conductivity measurements of GaAs/AlAs superlattices using a picosecond optical pump-and-probe technique"| In: Phys. Rev. B 59 (12 1999), pp. 8105-8113.

[4] Y. K. Koh, Y. Cao, D. G. Cahill, and D. Jena. "Heat-transport mechanisms in superlattices" In: Advanced Functional Materials 19.4 (2009), pp. 610-615.

[5] M. N. Luckyanova, J. Garg, K. Esfarjani, A. Jandl, M. T. Bulsara, A. J. Schmidt, A. J. Minnich, S. Chen, M. S. Dresselhaus, Z. Ren, et al. "Coherent phonon heat conduction in superlattices". In: Science 338.6109 (2012), pp. 936-939.

[6] A. Majumdar, K. Fushinobu, and K. Hijikata. "Effect of gate voltage on hotelectron and hot phonon interaction and transport in a submicrometer transistor". In: Journal of Applied Physics 77.12 (1995), pp. 6686-6694.

[7] D. G. Cahill, W. K. Ford, K. E. Goodson, G. D. Mahan, A. Majumdar, H. J. Maris, R. Merlin, and S. R. Phillpot. "Nanoscale thermal transport" In: Journal of Applied Physics 93.2 (2003), pp. 793-818.

[8] K. E. Goodson and Y. S. Ju. "Heat conduction in novel electronic films". In: Annual Review of Materials Science 29.1 (1999), pp. 261-293.

[9] W.-L. Ong, S. Majumdar, J. A. Malen, and A. J. H. McGaughey. "Coupling of organic and inorganic vibrational states and their thermal transport in nanocrystal arrays". In: The Journal of Physical Chemistry C 118.14 (2014), pp. 7288-7295.

[10] M. D. Losego, I. P. Blitz, R. A. Vaia, D. G. Cahill, and P. V. Braun. "Ultralow thermal conductivity in organoclay nanolaminates synthesized via simple self-assembly". In: Nano Letters 13.5 (2013), pp. 2215-2219.

[11] M. D. Losego, M. E. Grady, N. R. Sottos, D. G. Cahill, and P. V. Braun. "Effects of chemical bonding on heat transport across interfaces" In: Nat Mater 11.6 (June 2012), pp. 502-506.

[12] S. Majumdar, J. A. Sierra-Suarez, S. N. Schiffres, W.-L. Ong, I. C. Fred Higgs, A. J. H. McGaughey, and J. A. Malen. "Vibrational mismatch of metal leads controls thermal conductance of self-assembled monolayer junctions". In: Nano Letters 15.5 (2015), pp. 2985-2991.

[13] P. E. Hopkins. "Thermal transport across solid interfaces with nanoscale imperfections: effects of roughness, disorder, dislocations, and bonding on thermal boundary conductance". In: ISRN Mechanical Engineering 682586 (2013), p. 682586.

[14] B. C. Gundrum, D. G. Cahill, and R. S. Averback. "Thermal conductance of metal-metal interfaces" In: Phys. Rev. B 72 (24 2005), p. 245426.

[15] R. B. Wilson and D. G. Cahill. "Experimental validation of the interfacial form of the wiedemann-franz law" In: Phys. Rev Lett. 108 (25 2012), p. 255901.

[16] R. M. Costescu, M. A. Wall, and D. G. Cahill. "Thermal conductance of epitaxial interfaces" In: Phys. Rev. B 67 (5 2003), p. 054302 .

[17] J. C. Duda, C.-Y. P. Yang, B. M. Foley, R. Cheaito, D. L. Medlin, R. E. Jones, and P. E. Hopkins. "Influence of interfacial properties on thermal transport at gold:silicon contacts" In: Applied Physics Letters 102.8, 081902 (2013), p. 081902.

[18] H.-K. Lyeo and D. G. Cahill. "Thermal conductance of interfaces between highly dissimilar materials" In: Phys. Rev. B 73 (14 2006), p. 144301.

[19] R. J. Stoner, H. J. Maris, T. R. Anthony, and W. F. Banholzer. "Measurements of the Kapitza conductance between diamond and several metals" In: Phys. Rev. Lett. 68 (10 1992), pp. 1563-1566.

[20] Y. Jin, C. Shao, J. Kieffer, M. L. Falk, and M. Shtein. "Spatial nonuniformity in heat transport across hybrid material interfaces" In: Phys. Rev. B 90 (5 2014), p. 054306.

[21] P. L. Kapitza. "The study of heat transfer in Helium II". In: Zhurnal eksperimentalnoi i teoreticheskoi fiziki 11 (1941), pp. 1-31.

[22] E. T. Swartz and R. O. Pohl. "Thermal boundary resistance". In: Reviews of Modern Physics 61 (1989), pp. 605-668.

[23] E. Pop. "Energy dissipation and transport in nanoscale devices"| English. In: Nano Research 3.3 (2010), pp. 147-169.

[24] P. M. Norris and P. E. Hopkins. "Examining interfacial diffuse phonon scattering through transient thermoreflectance measurements of thermal boundary conductance" In: Journal of Heat Transfer 131.4 (Feb. 2009), p. 043207.

[25] R. J. Stoner and H. J. Maris. "Kapitza conductance and heat flow between solids at temperatures from 50 to 300 K" In: Phys. Rev. B 48 (22 1993), pp. 16373-16387.

[26] W. A. Little. "The transport of heat between dissimilar solids at low temperatures" In: Canadian Journal of Physics 37.3 (1959), pp. 334-349.

[27] M. L. Huberman and A. W. Overhauser. "Electronic Kapitza conductance at a diamond-Pb interface" In: Phys. Rev. B 50 (5 1994), pp. 2865-2873.

[28] A. V. Sergeev. "Electronic Kapitza conductance due to inelastic electron-boundary scattering"| In: Phys. Rev. B 58 (16 1998), R10199-R10202. 
[29] A Sergeev. "Inelastic electron-boundary scattering in thin films"] In: Physica B: Condensed Matter 263-264.0 (1999), pp. $217-$ 219.

[30] G. T. Hohensee, R. B. Wilson, and D. G. Cahill. "Thermal conductance of metal-diamond interfaces at high pressure"| In: Nat Commun 6 (Mar. 2015)

[31] P. E. Hopkins. "Effects of electron-boundary scattering on changes in thermoreflectance in thin metal films undergoing intraband excitations" In: Journal of Applied Physics 105.9, 093517 (2009).

[32] P. E. Hopkins, J. C. Duda, and P. M. Norris. "Anharmonic phonon interactions at interfaces and contributions to thermal boundary conductance" In: Journal of Heat Transfer 133.6 (Mar. 2011), p. 062401.

[33] J. C. Duda, P. M. Norris, and P. E. Hopkins. "On the linear temperature dependence of phonon thermal boundary conductance in the classical limit" In: Journal of Heat Transfer 133.7 (Apr. 2011), p. 074501.

[34] A. Majumdar and P. Reddy. "Role of electron-phonon coupling in thermal conductance of metal-nonmetal interfaces". In: Applied Physics Letters 84.23 (2004), pp. 4768-4770.

[35] P. E. Hopkins and P. M. Norris. "Substrate influence in electron-phonon coupling measurements in thin Au films"| In: Applied Surface Science 253.15 (2007), pp. 6289-6294.

[36] P. E. Hopkins, J. L. Kassebaum, and P. M. Norris. "Effects of electron scattering at metal-nonmetal interfaces on electronphonon equilibration in gold films"| In: Journal of Applied Physics 105.2, 023710 (2009), p. 023710.

[37] L. Guo, S. L. Hodson, T. S. Fisher, and X. Xu. "Heat transfer across metal-dielectric Interfaces during ultrafast-laser heating" In: Journal of Heat Transfer 134.4 (Feb. 2012), p. 042402.

[38] C. J. Vineis, A. Shakouri, A. Majumdar, and M. G. Kanatzidis. "Nanostructured thermoelectrics: Big efficiency gains from small features" In: Advanced Materials 22.36 (2010), pp. 3970-3980.

[39] R. Venkatasubramanian, E. Siivola, T. Colpitts, and B. O'Quinn. "Thin-film thermoelectric devices with high room-temperature figures of merit" In: Nature 413.6856 (Oct. 2001), pp. 597-602.

[40] B. H. Lee, B. Yoon, V. R. Anderson, and S. M. George. "Alucone alloys with tunable properties using alucone molecular layer deposition and $\mathrm{Al}_{2} \mathrm{O}_{3}$ atomic layer deposition". In: The Journal of Physical Chemistry C 116.5 (2012), pp. 3250-3257.

[41] B. Yoon, B. H. Lee, and S. M. George. "Molecular layer deposition of flexible, transparent and conductive hybrid molecular layer deposition of flexible, transparent and conductive hybrid organic-inorganic thin films". In: ECS Trans. 41.271 (2011), pp. 271-277.

[42] B. Yoon, B. H. Lee, and S. M. George. "Highly conductive and transparent hybrid organic-inorganic zincone thin films using atomic and molecular layer deposition". In: The Journal of Physical Chemistry C 116.46 (2012), pp. 24784-24791.

[43] T. Tynell, I. Terasaki, H. Yamauchi, and M. Karppinen. "Thermoelectric characteristics of (Zn,Al)O/hydroquinone superlattices" In: J. Mater. Chem. A 1 (43 2013), pp. 13619-13624.

[44] J. Liu, B. Yoon, E. Kuhlmann, M. Tian, J. Zhu, S. M. George, Y.-C. Lee, and R. Yang. "Ultralow thermal conductivity of atomic/molecular layer-deposited hybrid organic-Inorganic zincone thin films”. In: Nano Letters 13.11 (2013), pp. 5594-5599.

[45] Z. Zhang, J. Roger, D. Fournier, A. Boccara, and J. Wang. "Thermal diffusivity of amorphous semiconductor superlattice films" In: Thin Solid Films 186.2 (1990), pp. 361-366.

[46] B. Shen, Z. Zeng, C. Lin, and Z. Hu. "Thermal conductivity measurement of amorphous Si/SiGe multilayer films by 3 omega method" In: International Journal of Thermal Sciences 66.0 (2013), pp. 19-23.

[47] J. C. Duda, P. E. Hopkins, J. L. Smoyer, M. L. Bauer, T. S. English, C. B. Saltonstall, and P. M. Norris. "On the assumption of detailed balance in prediction of diffusive transmission probability during interfacial transport" In: Nanoscale and Microscale Thermophysical Engineering 14.1 (2010), pp. 21-33.

[48] I. M. Khalatnikov. "Heat exchange between a solid body and He II". In: Journal of Experimental and Theoretical Physics (Zhurnal Eksperimentalnoi i Teoreticheskoi Fiziki) 22 (1952), p. 687.

[49] P. Reddy, K. Castelino, and A. Majumdar. "Diffuse mismatch model of thermal boundary conductance using exact phonon dispersion". In: Applied Physics Letters 87.21, 211908 (2005), p. 211908.

[50] R. Prasher. "Acoustic mismatch model for thermal contact resistance of van der Waals contacts". In: Applied Physics Letters 94.4, 041905 (2009).

[51] J. C. Duda, J. L. Smoyer, P. M. Norris, and P. E. Hopkins. "Extension of the diffuse mismatch model for thermal boundary conductance between isotropic and anisotropic materials". In: Applied Physics Letters 95.3, 031912 (2009).

[52] P. E. Hopkins and P. M. Norris. "Relative contributions of inelastic and elastic diffuse phonon scattering to thermal boundary conductance across solid interfaces" In: Journal of Heat Transfer 131.2 (Jan. 2009), pp. 022402-022402.

[53] P. E. Hopkins, T. Beechem, J. C. Duda, K. Hattar, J. F. Ihlefeld, M. A. Rodriguez, and E. S. Piekos. "Influence of anisotropy on thermal boundary conductance at solid interfaces" In: Phys. Rev. B 84 (12 2011), p. 125408.

[54] A. Giri, B. M. Foley, and P. E. Hopkins. "Influence of hot electron scattering and electron-phonon interactions on thermal boundary conductance at metal/nonmetal interfaces". In: Journal of Heat Transfer 136.9 (June 2014), p. 092401.

[55] A. Giri, J. L. Braun, and P. E. Hopkins. "Implications of interfacial bond strength on the spectral contributions to thermal boundary conductance across solid, liquid, and gas interfaces: A molecular dynamics study". In: The Journal of Physical Chemistry C 120.43 (Nov. 2016), pp. 24847-24856.

[56] S. I. Anisimov, B. L. Kapeliovich, and T. L. Perelman. "Electron emission from metal surfaces exposed to ultrashort laser pulses". In: Sov. Phys. JETP 39 (1974), p. 375.

[57] J. Hohlfeld, S.-S. Wellershoff, J. Güdde, U. Conrad, V. Jähnke, and E. Matthias. "Electron and lattice dynamics following optical excitation of metals" In: Chemical Physics 251.1-3 (2000), pp. 237 -258.

[58] Z. Lin, L. V. Zhigilei, and V. Celli. "Electron-phonon coupling and electron heat capacity of metals under conditions of strong electron-phonon nonequilibrium" In: Phys. Rev. B 77 (7 2008), p. 075133.

[59] T. Q. Qiu and C. L. Tien. "Size Effects on Nonequilibrium Laser Heating of Metal Films" In: Journal of Heat Transfer 115.4 (Nov. 1993), pp. 842-847.

[60] G. Grimvall. Selected Topics in Solid State Physics. Ed. by E. Whohlfarth. New York: North-Holland, 1981.

[61] M. Kaganov, I. Lifshitz, and L. V. Tanatarov. "Relaxation between electrons and the crystalline lattice". In: Sov. Phys. JETP 4.2 (1957), pp. 173-178. 
[62] P. E. Hopkins, T. E. Beechem, J. C. Duda, J. L. Smoyer, and P. M. Norris. "Effects of subconduction band excitations on thermal conductance at metal-metal interfaces" In: Applied Physics Letters 96.1, 011907 (2010), p. 011907.

[63] J. C. Duda, T. E. Beechem, J. L. Smoyer, P. M. Norris, and P. E. Hopkins. "Role of dispersion on phononic thermal boundary conductance" In: Journal of Applied Physics 108.7, 073515 (2010), p. 073515.

[64] R. Cheaito, K. Hattar, J. T. Gaskins, A. K. Yadav, J. C. Duda, T. E. Beechem, J. F. Ihlefeld, E. S. Piekos, J. K. Baldwin, A. Misra, et al. "Thermal flux limited electron Kapitza conductance in copper-niobium multilayers"| In: Applied Physics Letters 106.9, 093114 (2015), pp. - .

[65] S. Plimpton. "Fast parallel algorithms for short-range molecular dynamics"] In: Journal of Computational Physics 117.1 (1995), pp. 1-19.

[66] W. G. Hoover. "Canonical dynamics: Equilibrium phase-space distributions"] In: Phys. Rev. A 31 (3 1985), pp. $1695-1697$.

[67] J. C. Duda, T. S. English, E. S. Piekos, W. A. Soffa, L. V. Zhigilei, and P. E. Hopkins. "Implications of cross-species interactions on the temperature dependence of Kapitza conductance" In: Phys. Rev. B 84 (19 2011), p. 193301.

[68] M. P. Allen and D. J. Tildesley. Computer simulation of liquids (Oxford Science Publications). Reprint. Oxford science publications. Oxford University Press, June 1989.

[69] K. Sääskilahti, J. Oksanen, J. Tulkki, and S. Volz. "Role of anharmonic phonon scattering in the spectrally decomposed thermal conductance at planar interfaces" In: Phys. Rev. B 90 (13 2014), p. 134312.

[70] K. Sääskilahti, J. Oksanen, R. P. Linna, and J. Tulkki. "Thermal conduction and interface effects in nanoscale Fermi-Pasta-Ulam conductors" In: Phys. Rev. E 86 (3 2012), p. 031107.

[71] Z.-Y. Ong and E. Pop. "Frequency and polarization dependence of thermal coupling between carbon nanotubes and $\mathrm{SiO} 2 "$ ". In: Journal of Applied Physics 108.10, 103502 (2010), p. 103502.

[72] G. Domingues, S. Volz, K. Joulain, and J.-J. Greffet. "Heat transfer between two nanoparticles through near field interaction" In: Phys. Rev. Lett. 94 (8 2005), p. 085901.

[73] Y. Zhou and M. Hu. "Quantitatively analyzing phonon spectral contribution of thermal conductivity based on nonequilibrium molecular dynamics simulations. II. From time Fourier transform"| In: Phys. Rev. B 92 (19 2015), p. 195205.

[74] Y. Zhou, X. Zhang, and M. Hu. "Quantitatively analyzing phonon spectral contribution of thermal conductivity based on nonequilibrium molecular dynamics simulations. I. From space Fourier transform" In: Phys. Rev. B 92 (19 2015), p. 195204.

[75] J. E. Turney, E. S. Landry, A. J. H. McGaughey, and C. H. Amon. "Predicting phonon properties and thermal conductivity from anharmonic lattice dynamics calculations and molecular dynamics simulations" In: Phys. Rev. B 79 (6 2009), p. 064301.

[76] P. E. Hopkins, R. J. Stevens, and P. M. Norris. "Influence of Inelastic Scattering at Metal-Dielectric Interfaces" In: Journal of Heat Transfer 130.2 (Feb. 2008), pp. 022401-022401.

[77] P. E. Hopkins. "Multiple phonon processes contributing to inelastic scattering during thermal boundary conductance at solid interfaces" In: Journal of Applied Physics 106.1, 013528 (2009), p. 013528.

[78] J. C. Duda, P. E. Hopkins, T. E. Beechem, J. L. Smoyer, and P. M. Norris. "Inelastic phonon interactions at solid-graphite interfaces" In: Superlattices and Microstructures 47.4 (2010), pp. $550-555$.

[79] K. Gordiz and A. Henry. "A formalism for calculating the modal contributions to thermal interface conductance" In: New Journal of Physics 17.10 (2015), p. 103002.

[80] G. L. Eesley. "Observation of Nonequilibrium Electron Heating in Copper"' In: Phys. Rev. Lett. 51 (23 1983), pp. $2140-2143$.

[81] A. J. Schmidt. "Optical characterization of thermal transport from the nanoscale to the macroscale". PhD thesis. Massachusetts Institute Of Technology, 2008.

[82] R. Cheaito. "The role of size effects on the thermal conductivity of thin film alloys and superlattices". PhD thesis. School of Engineering and Applied Science, University of Virginia, 2015.

[83] D. G. Cahill. "Analysis of heat flow in layered structures for time-domain thermoreflectance Analysis of heat flow in layered structures for time-domain thermoreflectance". In: Review of Scientific Instruments 75 (2004), pp. 5119-5122.

[84] A. J. Schmidt, X. Chen, and G. Chen. "Pulse accumulation, radial heat conduction, and anisotropic thermal conductivity in pump-probe transient thermoreflectance". In: Rev Sci Instrum 79.11 (2008), p. 114902.

[85] P. E. Hopkins, J. R. Serrano, L. M. Phinney, S. P. Kearney, T. W. Grasser, and C. T. Harris. "Criteria for cross-plane dominated thermal transport in multilayer thin film systems during modulated laser heating"' In: Journal of Heat Transfer 132.8 (May 2010), p. 081302.

[86] R. Trebino, K. W. DeLong, D. N. Fittinghoff, J. N. Sweetser, M. A. Krumbgel, B. A. Richman, and D. J. Kane. "Measuring ultrashort laser pulses in the time-frequency domain using frequency-resolved optical gating"| In: Review of Scientific Instruments 68.9 (1997), pp. 3277-3295.

[87] S. D. Brorson, A. Kazeroonian, J. S. Moodera, D. W. Face, T. K. Cheng, E. P. Ippen, M. S. Dresselhaus, and G. Dresselhaus. "Femtosecond room-temperature measurement of the electron-phonon coupling constant $\gamma$ in metallic superconductors" In: Phys. Rev. Lett. 64 (18 1990), pp. 2172-2175.

[88] P. M. Norris, A. P. Caffrey, R. J. Stevens, J. M. Klopf, J. James T. McLeskey, and A. N. Smith. "Femtosecond pump-probe nondestructive examination of materials (invited)" In: Review of Scientific Instruments 74.1 (2003), pp. 400-406.

[89] C. Thomsen, J. Strait, Z. Vardeny, H. J. Maris, J. Tauc, and J. J. Hauser. "Coherent phonon generation and detection by picosecond light pulses"' In: Phys. Rev. Lett. 53 (10 1984), pp. 989-992.

[90] C. Thomsen, H. T. Grahn, H. J. Maris, and J. Tauc. "Surface generation and detection of phonons by picosecond light pulses" In: Phys. Rev. B 34 (6 1986), pp. 4129-4138.

[91] J. Alvarez-Quintana, E. Martínez, E. Pérez-Tijerina, S. A. Pérez-García, and J. Rodríguez-Viejo. "Temperature dependent thermal conductivity of polycrystalline ZnO films". In: Journal of Applied Physics 107.6, 063713 (2010), p. 063713.

[92] J. P. Feser and D. G. Cahill. "Probing anisotropic heat transport using time-domain thermoreflectance with offset laser spots" In: Review of Scientific Instruments 83.10, 104901 (2012), pp. - .

[93] D. G. Cahill, P. V. Braun, G. Chen, D. R. Clarke, S. Fan, K. E. Goodson, P. Keblinski, W. P. King, G. D. Mahan, A. Majumdar, et al. "Nanoscale thermal transport. II. 2003-2012". In: Applied Physics Reviews 1.1, 011305 (2014), p. 011305.

[94] R. B. Wilson, J. P. Feser, G. T. Hohensee, and D. G. Cahill. "Two-channel model for nonequilibrium thermal transport in pump-probe experiments" In: Phys. Rev. B 88 (14 2013), p. 144305. 
[95] T. Qiu and C. Tien. "Short-pulse laser heating on metals" In: International Journal of Heat and Mass Transfer 35.3 (1992), pp. 719-726.

[96] T. Q. Qiu and C. L. Tien. "Heat transfer mechanisms during short-pulse laser heating of metals" In: Journal of Heat Transfer 115.4 (Nov. 1993), pp. 835-841.

[97] P. E. Hopkins, L. M. Phinney, and J. R. Serrano. "Re-examining electron-fermi relaxation in gold films with a nonlinear thermoreflectance model" In: Journal of Heat Transfer 133.4 (Jan. 2011), pp. 044505-044505.

[98] M. I. Markovic and A. D. Rakic. "Determination of the reflection coefficients of laser light of wavelengths $\lambda \varepsilon(0.22 \mu \mathrm{m}, 200$ $\mu \mathrm{m})$ from the surface of aluminum using the Lorentz-Drude model" In: Appl. Opt. 29.24 (1990), pp. 3479-3483.

[99] R. E. Hummel. Electronic Properties of Materials. 3rd. Springer-Verlag, New York, 2001.

[100] P. E. Hopkins, J. C. Duda, B. Kaehr, X. Wang Zhou, C.-Y. Peter Yang, and R. E. Jones. "Ultrafast and steady-state laser heating effects on electron relaxation and phonon coupling mechanisms in thin gold films" In: Applied Physics Letters 103.21, 211910 (2013), p. 211910

[101] J. K. Chen, W. P. Latham, and J. E. Beraun. "The role of electronÄìphonon coupling in ultrafast laser heating"| In: Journal of Laser Applications 17.1 (2005), pp. 63-68.

[102] M. Kaveh and N. Wiser. "Electron-electron scattering in conducting materials". In: Advances in Physics 33.4 (1984), pp. 257372.

[103] X. Y. Wang, D. M. Riffe, Y.-S. Lee, and M. C. Downer. "Time-resolved electron-temperature measurement in a highly excited gold target using femtosecond thermionic emission" In: Phys. Rev. B 50 (11 1994), pp. 8016-8019.

[104] W. Wang and D. G. Cahill. "Limits to thermal transport in nanoscale metal bilayers due to weak electron-phonon coupling in Au and Cu" In: Phys. Rev. Lett. 109 (17 2012), p. 175503.

[105] A. Giri, J. T. Gaskins, B. F. Donovan, C. Szwejkowski, R. J. Warzoha, M. A. Rodriguez, J. Ihlefeld, and P. E. Hopkins. "Mechanisms of nonequilibrium electron-phonon coupling and thermal conductance at interfaces"| In: Journal of Applied Physics 117.10, 105105 (2015), p. 105105.

[106] T. H. Geballe and W. F. Giauque. "The heat capacity and entropy of gold from 15 to $300^{\circ}$ K.1" In: Journal of the American Chemical Society 74.9 (1952), pp. 2368-2369.

[107] L. R. Holland. "Physical Properties of Titanium. III. The Specific Heat". In: Journal of Applied Physics 34.8 (1963), pp. 23502357.

[108] M Born and E Wolf. Principles of Optics. 7th ed. Cambridge University Press, 1999.

[109] F. Abeles. Advanced optical techniques. Ed. by A. V. Heel. 145-188. Amsterdam: North-Holland Publishing Co., 1967.

[110] P. E. Hopkins. "Influence of electron-boundary scattering on thermoreflectance calculations after intra- and interband transitions induced by short-pulsed laser absorption"| In: Phys. Rev. B 81 (3 2010), p. 035413.

[111] A. N. Smith and P. M. Norris. "Influence of intraband transitions on the electron thermoreflectance response of metals" In: Applied Physics Letters 78.9 (2001), pp. 1240-1242.

[112] P. E. Hopkins. "Thermoreflectance dependence on Fermi surface electron number density perturbations" In: Applied Physics Letters 96.4, 041901 (2010), p. 041901.

[113] P. E. Hopkins and D. A. Stewart. "Contribution of d-band electrons to ballistic transport and scattering during electron-phonon nonequilibrium in nanoscale Au films using an ab initio density of states". In: Journal of Applied Physics 106.5, 053512 (2009).

[114] A. Schmidt, M. Chiesa, X. Chen, and G. Chen. "An optical pump-probe technique for measuring the thermal conductivity of liquids" In: Review of Scientific Instruments 79.6, 064902 (2008), pp. - .

[115] G. Tas and H. J. Maris. "Electron diffusion in metals studied by picosecond ultrasonics" In: Phys. Rev. B 49 (21 1994), pp. 15046-15054.

[116] B. Y. Mueller and B. Rethfeld. "Relaxation dynamics in laser-excited metals under nonequilibrium conditions"| In: Phys. Rev. B 87 (3 2013), p. 035139.

[117] G.-M. Choi, R. B. Wilson, and D. G. Cahill. "Indirect heating of Pt by short-pulse laser irradiation of Au in a nanoscale Pt/Au bilayer" In: Phys. Rev. B 89 (6 2014), p. 064307.

[118] B. Singh and N. Surplice. "The electrical resistivity and resistance-temperature characteristics of thin titanium films" In: Thin Solid Films 10.2 (1972), pp. $243-253$.

[119] A. Giri, J. T. Gaskins, B. M. Foley, R. Cheaito, and P. E. Hopkins. "Experimental evidence of excited electron number density and temperature effects on electron-phonon coupling in gold films" In: Journal of Applied Physics 117.4, 044305 (2015), p. 044305.

[120] A. P. Caffrey, P. E. Hopkins, J. M. Klopf, and P. M. Norris. "Thin film non-noble transition metal thermophysical properties." In: Microscale Thermophysical Engineering 9.4 (2005), pp. $365-377$.

[121] D. G. Cahill, S. K. Watson, and R. O. Pohl. "Lower limit to the thermal conductivity of disordered crystals"] In: Phys. Rev. B 46 (10 1992), pp. 6131-6140.

[122] D. E. Gray. American Institute of Physics handbook. 3rd. New York: McGraw-Hil, 1972.

[123] G. Tas and H. J. Maris. "Picosecond ultrasonic study of phonon reflection from solid-liquid interfaces"] In: Phys. Rev. B 55 (3 1997), pp. 1852-1857.

[124] P. E. Hopkins, M. Baraket, E. V. Barnat, T. E. Beechem, S. P. Kearney, J. C. Duda, J. T. Robinson, and S. G. Walton. "Manipulating thermal conductance at metal-graphene contacts via chemical functionalization". In: Nano Letters 12.2 (2012), pp. 590595.

[125] R. Cheaito, J. T. Gaskins, M. E. Caplan, B. F. Donovan, B. M. Foley, A. Giri, J. C. Duda, C. J. Szwejkowski, C. Constantin, H. J. Brown-Shaklee, et al. "Thermal boundary conductance accumulation and interfacial phonon transmission: Measurements and theory" In: Phys. Rev. B 91 (3 2015), p. 035432.

[126] A. Giri, J.-P. Niemelä, T. Tynell, J. T. Gaskins, B. F. Donovan, M. Karppinen, and P. E. Hopkins. “Heat-transport mechanisms in molecular building blocks of inorganic/organic hybrid superlattices"] In: Phys. Rev. B 93 (11 2016), p. 115310.

[127] A. Giri, J.-P. Niemelä, C. J. Szwejkowski, M. Karppinen, and P. E. Hopkins. "Reduction in thermal conductivity and tunable heat capacity of inorganic/organic hybrid superlattices” In: Phys. Rev. B 93 (2016), p. 024201. 
[128] G. Chen. Nanoscale Energy Transport and Conversion: A Parallel Treatment of Electrons, Molecules, Phonons, and Photons (MIT-Pappalardo Series in Mechanical Engineering). Oxford University Press, USA, Mar. 2005.

[129] R. E. Jones, J. C. Duda, X. W. Zhou, C. J. Kimmer, and P. E. Hopkins. "Investigation of size and electronic effects on Kapitza conductance with non-equilibrium molecular dynamics"| In: Applied Physics Letters 102.18, 183119 (2013), p. 183119.

[130] N. Mingo, D. Hauser, N. P. Kobayashi, M. Plissonnier, and A. Shakouri. "Nanoparticle-in-alloy approach to efficient thermoelectrics: Silicides in SiGe”. In: Nano Letters 9.2 (2009), pp. 711-715.

[131] S. J. Poon and K. Limtragool. "Nanostructure model of thermal conductivity for high thermoelectric performance"' In: Journal of Applied Physics 110.11, 114306 (2011), p. 114306.

[132] J. Tang, H.-T. Wang, D. H. Lee, M. Fardy, Z. Huo, T. P. Russell, and P. Yang. "Holey Silicon as an Efficient Thermoelectric Material". In: Nano Letters 10.10 (2010), pp. 4279-4283.

[133] Y. Chujo. "Organic-inorganic hybrid materials" In: Current Opinion in Solid State and Materials Science 1.6 (1996), pp. 806 -811 .

[134] P. Judeinstein and C. Sanchez. "Hybrid organic-inorganic materials: a land of multidisciplinarity" In: J. Mater. Chem. 6 (4 1996), pp. 511-525.

[135] K.-H. Yoon, K.-S. Han, and M.-M. Sung. "Fabrication of a new type of organic-inorganic hybrid superlattice films combined with titanium oxide and polydiacetylene". In: Nanoscale Research Letters 7.1 (2012), p. 71.

[136] T. Tynell, A. Giri, J. Gaskins, P. E. Hopkins, P. Mele, K. Miyazaki, and M. Karppinen. "Efficiently suppressed thermal conductivity in $\mathrm{ZnO}$ thin films via periodic introduction of organic layers"' In: J. Mater. Chem. A 2 (31 2014), p. 12150

[137] J.-P. Niemelä, A. Giri, P. E. Hopkins, and M. Karppinen. “Ultra-low thermal conductivity in $\mathrm{TiO}_{2}: C$ superlattices" In: J. Mater. Chem. A 3 (2015), p. 11527.

[138] W.-L. Ong, S. M. Rupich, D. V. Talapin, A. J. H. McGaughey, and J. A. Malen. "Surface chemistry mediates thermal transport in three-dimensional nanocrystal arrays". In: Nat Mater 12.5 (May 2013), pp. 410-415.

[139] J. P. Feser, E. M. Chan, A. Majumdar, R. A. Segalman, and J. J. Urban. "Ultralow thermal conductivity in polycrystalline CdSe thin films with controlled grain size". In: Nano Letters 13.5 (2013), pp. 2122-2127.

[140] J. C. Duda, P. E. Hopkins, Y. Shen, and M. C. Gupta. "Exceptionally low thermal conductivities of films of the fullerene derivative PCBM" In: Phys. Rev. Lett. 110 (1 2013), p. 015902.

[141] R. Y. Wang, R. A. Segalman, and A. Majumdar. "Room temperature thermal conductance of alkanedithiol self-assembled monolayers" In: Applied Physics Letters 89.17, 173113 (2006), p. 173113.

[142] Z. Wang, J. A. Carter, A. Lagutchev, Y. K. Koh, N.-H. Seong, D. G. Cahill, and D. D. Dlott. "Ultrafast flash thermal conductance of molecular chains". In: Science 317.5839 (2007), pp. 787-790.

[143] Z. Wang, D. G. Cahill, J. A. Carter, Y. K. Koh, A. Lagutchev, N.-H. Seong, and D. D. Dlott. "Ultrafast dynamics of heat flow across molecules". In: Chemical Physics 350.1Äì3 (2008), pp. 31 -44.

[144] P. J. O'Brien, S. Shenogin, J. Liu, P. K. Chow, D. Laurencin, P. H. Mutin, M. Yamaguchi, P. Keblinski, and G. Ramanath. "Bonding-induced thermal conductance enhancement at inorganic heterointerfaces using nanomolecular monolayers"| In: Nat Mater 12.2 (Feb. 2013), pp. 118-122.

[145] Y. Jin, C. Shao, J. Kieffer, K. P. Pipe, and M. Shtein. "Origins of thermal boundary conductance of interfaces involving organic semiconductors" In: Journal of Applied Physics 112.9, 093503 (2012), p. 093503.

[146] P. Sundberg and M. Karppinen. "Organic and inorganic Äiorganic thin film structures by molecular layer deposition: A review". In: Beilstein Journal of Nanotechnology 5 (2014), pp. 1104-1136.

[147] J.-P. Niemelä and M. Karppinen. "Tunable optical properties of hybrid inorganic-organic $\left[\left(\mathrm{TiO}_{2}\right) \mathrm{m}\left(\mathrm{Ti}-\mathrm{O}-\mathrm{C}_{6} \mathrm{H}_{4}-\mathrm{O}-\right) \mathrm{k}\right] \mathrm{n}$ superlattice thin films" In: Dalton Trans. 44 (2 2015), pp. 591-597.

[148] V. Holý, U. Pietsch, and T. Baumbach. High-Resolution X-Ray Scattering from Thin Films and Multilayers. p. 127. Berlin: Springer Berlin Heidelberg, 1999.

[149] C. Tasaki, N. Oka, T. Yagi, N. Taketoshi, T. Baba, T. Kamiyama, S. ichi Nakamura, and Y. Shigesato. "Thermophysical properties of transparent conductive Nb-doped $\mathrm{TiO}_{2}$ Films" In: Japanese Journal of Applied Physics 51.3R (2012), p. 035802.

[150] S.-M. Lee, D. G. Cahill, and T. H. Allen. "Thermal conductivity of sputtered oxide films" In: Phys. Rev. B 52 (1 1995), pp. 253257.

[151] Y. S. Touloukian, R. W. Powell, C. Y. Ho, and P. G. Klemens. Thermophysical Properties of Matter - The TPRC Data Series. Volume 2. Thermal Conductivity - Nonmetallic Solids. Vol. 2. IFI/Plenum, New York, 1970.

[152] Y. Ding and B. Xiao. "Anisotropic elasticity, sound velocity and thermal conductivity of $\mathrm{TiO}_{2}$ polymorphs from first principles calculation" In: Computational Materials Science 82.0 (2014), pp. $202-218$.

[153] C. Klingshirn, A. Waag, A. Hoffmann, and J. Geurts. Zinc Oxide: From Fundamental Properties Towards Novel Applications. Vol. 120. Springer, 2010.

[154] J. Serrano, F. Manjón, A. Romero, A. Ivanov, M. Cardona, R. Lauck, A. Bosak, and M. Krisch. "Phonon dispersion relations of zinc oxide: Inelastic neutron scattering and ab initio calculations" In: Phys. Rev. B 81 (17 2010), p. 174304.

[155] J. Ravichandran, A. K. Yadav, R. Cheaito, P. B. Rossen, A. Soukiassian, S. J. Suresha, J. C. Duda, B. M. Foley, C.-H. Lee, Y. Zhu, et al. "Crossover from incoherent to coherent phonon scattering in epitaxial oxide superlattices"| In: Nat Mater 13.2 (Feb. 2014), pp. 168-172.

[156] P. M. Norris, N. Q. Le, and C. H. Baker. "Tuning phonon transport: From interfaces to nanostructures" In: Journal of Heat Transfer 135.6 (May 2013), pp. 061604-061604.

[157] G. Chen and T. Zeng. "Nonequilibrium phonon and electron transport in heterostructures and superlattices". In: Microscale Thermophysical Engineering 5.2 (2001), pp. 71-88.

[158] R. M. Costescu, D. G. Cahill, F. H. Fabreguette, Z. A. Sechrist, and S. M. George. "Ultra-Low Thermal Conductivity in W/A12O3 Nanolaminates". In: Science 303.5660 (2004), pp. 989-990.

[159] P. E. Hopkins, J. C. Duda, C. W. Petz, and J. A. Floro. "Controlling thermal conductance through quantum dot roughening at interfaces" In: Phys. Rev. B 84 (3 2011), p. 035438.

[160] J. C. Duda and P. E. Hopkins. "Systematically controlling Kapitza conductance via chemical etching"| In: Applied Physics Letters 100.11, 111602 (2012), pp. - . 
[161] P. E. Hopkins, L. M. Phinney, J. R. Serrano, and T. E. Beechem. "Effects of surface roughness and oxide layer on the thermal boundary conductance at aluminum/silicon interfaces" In: Phys. Rev. B 82 (8 2010), p. 085307.

[162] A. J. Karttunen, T. Tynell, and M. Karppinen. "Atomic-level structural and electronic properties of hybrid inorganic-organic ZnO:hydroquinone superlattices fabricated by ALD/MLD”. In: The Journal of Physical Chemistry C 119.23 (2015), pp. 1310513114.

[163] A. Strachan, A. C. T. van Duin, D. Chakraborty, S. Dasgupta, and W. A. Goddard. "Shock waves in high-energy materials: The initial chemical events in nitramine RDX" In: Phys. Rev. Lett. 91 (9 2003), p. 098301.

[164] J. C. Duda, C. B. Saltonstall, P. M. Norris, and P. E. Hopkins. "Assessment and prediction of thermal transport at solidÄìselfassembled monolayer junctions" In: The Journal of Chemical Physics 134.9, 094704 (2011), pp. - .

[165] T. Meier, F. Menges, P. Nirmalraj, H. Hölscher, H. Riel, and B. Gotsmann. "Length-dependent thermal transport along molecular chains" In: Phys. Rev. Lett. 113 (6 2014), p. 060801.

[166] D. Segal, A. Nitzan, and P. Hänggi. "Thermal conductance through molecular wires" In: The Journal of Chemical Physics 119.13 (2003), pp. 6840-6855.

[167] H. Jin, G. Marin, A. Giri, T. Tynell, E. Kontturi, T. Tammelin, P. E. Hopkins, and M. Karppinen. "Reduced thermal conductivity in ZnO:nanocellulose hybrid thin films". In: under review (2016).

[168] Z.-G. Mei, Y. Wang, S.-L. Shang, and Z.-K. Liu. "First-principles study of lattice dynamics and thermodynamics of $\mathrm{TiO}_{2}$ polymorphs". In: Inorganic Chemistry 50.15 (2011), pp. 6996-7003.

[169] O. Madelung, U. Rössler, and M. Schulz. "Landolt-Börnstein - group III condensed matter". In: II-VI and I-VII Compounds; Semimagnetic Compounds. Vol. 41B. Springer Berlin Heidelberg, 1999, pp. 1-5.

[170] A. Giri, J. L. Braun, and P. E. Hopkins. "Effect of crystalline/amorphous interfaces on thermal transport across confined thin films and superlattices" In: Journal of Applied Physics 119.23, 235305 (2016).

[171] A. Giri, P. E. Hopkins, J. G. Wessel, and J. C. Duda. "Kapitza resistance and the thermal conductivity of amorphous superlattices" In: Journal of Applied Physics 118.16, 165303 (2015), p. 165303.

[172] F. Jolly, F. Rochet, G. Dufour, C. Grupp, and A. Taleb-Ibrahimi. "Oxidized silicon surfaces studied by high resolution Si 2p core-level photoelectron spectroscopy using synchrotron radiation" In: Journal of Non-Crystalline Solids 280.1 Aì3 (2001). 3rd Symp. on $\mathrm{SiO}_{2}$ and Advanced Dielectrics, pp. $150-155$.

[173] T. S. English, J. C. Duda, J. L. Smoyer, D. A. Jordan, P. M. Norris, and L. V. Zhigilei. "Enhancing and tuning phonon transport at vibrationally mismatched solid-solid interfaces" In: Phys. Rev. B 85 (3 2012), p. 035438.

[174] E. S. Landry and A. J. H. McGaughey. "Effect of film thickness on the thermal resistance of confined semiconductor thin films" In: Journal of Applied Physics 107.1, 013521 (2010), p. 013521.

[175] B. Abeles and T. Tiedje. “Amorphous semiconductor superlattices" In: Phys. Rev. Lett. 51 (21 1983), pp. 2003-2006.

[176] N. Maley and J. S. Lannin. "Phonons in amorphous semiconductor superlattices" In: Phys. Rev. B 31 (8 1985), pp. 5577-5579.

[177] P. Santos, M. Hundhausen, and L. Ley. "Observation of folded-zone acoustical phonons by Raman scattering in amorphous Si-SiN ${ }_{\mathrm{x}}$ superlattices"| In: Phys. Rev. B 33 (2 1986), pp. 1516-1518.

[178] O. Koblinger, J. Mebert, E. Dittrich, S. Döttinger, W. Eisenmenger, P. V. Santos, and L. Ley. "Phonon stop bands in amorphous superlattices" In: Phys. Rev. B 35 (17 1987), pp. 9372-9375.

[179] P. B. Allen and J. L. Feldman. "Thermal conductivity of disordered harmonic solids" In: Phys. Rev. B 48 (17 1993), pp. 1258112588 .

[180] P. B. Allen, J. L. Feldman, J. Fabian, and F. Wooten. "Diffusons, locons and propagons: Character of atomie yibrations in amorphous Si" In: Philosophical Magazine Part B 79.11-12 (1999), pp. 1715-1731.

[181] J. L. Feldman, M. D. Kluge, P. B. Allen, and F. Wooten. "Thermal conductivity and localization in glasses: Numerical study of a model of amorphous silicon" In: Phys. Rev. B 48 (17 1993), pp. 12589-12602.

[182] J. M. Larkin and A. J. H. McGaughey. "Thermal conductivity accumulation in amorphous silica and amorphous silicon" In: Phys. Rev. B 89 (14 2014), p. 144303

[183] P. Santos and L. Ley. "Phonons in amorphous superlattices"] In: Superlattices and Microstructures 5.1 (1989), pp. 43 -50.

[184] D. Hondongwa, L. Olasov, B. Daly, S. King, and J. Bielefeld. "Thermal conductivity and sound velocity measurements of plasma enhanced chemical vapor deposited a-SiC:H thin films" In: Thin Solid Films 519.22 (2011), pp. 7895-7898.

[185] M. R. Baklanov, M. Van Hove, G. Mannaert, S. Vanhaelemeersch, H. Bender, T. Conard, and K. Maex. "Low temperature oxidation and selective etching of chemical vapor deposition a-SiC:H films" In: Journal of Vacuum Science Technology B 18.3 (2000), pp. 1281-1287.

[186] J. Chen, S. W. King, E. Muthuswamy, A. Koryttseva, D. Wu, and A. Navrotsky. "Thermodynamic stability of low-k amorphous SiOCH Dielectric Films"| In: Journal of the American Ceramic Society 99.8 (2016), pp. 2752-2759.

[187] J. Tersoff. "New empirical approach for the structure and energy of covalent systems" In: Phys. Rev. B 37 (12 1988), pp. 69917000 .

[188] J. Tersoff. "Modeling solid-state chemistry: Interatomic potentials for multicomponent systems" In: Phys. Rev. B 39 (8 1989), pp. 5566-5568

[189] E. S. Landry and A. J. H. McGaughey. "Thermal boundary resistance predictions from molecular dynamics simulations and theoretical calculations" In: Phys. Rev. B 80 (16 2009), p. 165304

[190] Z. Liang and P. Keblinski. "Finite-size effects on molecular dynamics interfacial thermal-resistance predictions"| In: Phys. Rev. B 90 (7 2014), p. 075411.

[191] H. Acharya, N. J. Mozdzierz, P. Keblinski, and S. Garde. "How chemistry, nanoscale roughness, and the direction of heat flow affect thermal conductance of solid-water interfaces". In: Industrial and Engineering Chemistry Research 51.4 (2012), pp. 1767-1773.

[192] B. H. Kim, A. Beskok, and T. Cagin. "Molecular dynamics simulations of thermal resistance at the liquid-solid interface". In: The Journal of Chemical Physics 129.17, 174701 (2008), p. 174701.

[193] Z. Liang, W. Evans, and P. Keblinski." "Equilibrium and nonequilibrium molecular dynamics simulations of thermal conductance at solid-gas interfaces" In: Phys. Rev. E 87 (2 2013), p. 022119. 
[194] Z. Liang, W. Evans, T. Desai, and P. Keblinski. "Improvement of heat transfer efficiency at solid-gas interfaces by selfassembled monolayers". In: Applied Physics Letters 102.6, 061907 (2013).

[195] Z. Liang and P. Keblinski. "Parametric studies of the thermal and momentum accommodation of monoatomic and diatomic gases on solid surfaces"| In: International Journal of Heat and Mass Transfer 78 (2014), pp. 161 -169.

[196] C. Shao and H. Bao. "A molecular dynamics investigation of heat transfer across a disordered thin film" In: International Journal of Heat and Mass Transfer 85.0 (2015), pp. 33-40.

[197] Y. Chalopin, K. Esfarjani, A. Henry, S. Volz, and G. Chen. "Thermal interface conductance in Si/Ge superlattices by equilibrium molecular dynamics". In: Phys. Rev. B 85 (19 2012), p. 195302.

[198] S. Merabia and K. Termentzidis. "Thermal conductance at the interface between crystals using equilibrium and nonequilibrium molecular dynamics" In: Phys. Rev. B 86 (9 2012), p. 094303.

[199] B. Peng, W. He, X. Hao, Y. Chen, and Y. Liu. "Interfacial thermal conductance and thermal accommodation coefficient of evaporating thin liquid films: A molecular dynamics study" In: Computational Materials Science 87 (2014), pp. 260 -266.

[200] M. Li, J. Zhang, X. Hu, and Y. Yue. "Thermal transport across graphene/SiC interface: effects of atomic bond and crystallinity of substrate" In: Applied Physics A 119.2 (2015), pp. 415-424.

[201] T. Beechem and P. E. Hopkins. "Predictions of thermal boundary conductance for systems of disordered solids and interfaces". In: Journal of Applied Physics 106.12, 124301 (2009).

[202] F. H. Stillinger and T. A. Weber. "Computer simulation of local order in condensed phases of silicon"] In: Phys. Rev. B 31 (8 1985), pp. 5262-5271.

[203] K. Ding and H. C. Andersen. "Molecular-dynamics simulation of amorphous germanium" In: Phys. Rev. B 34 (10 1986), pp. 6987-6991.

[204] M. Laradji, D. P. Landau, and B. Dünweg. "Structural properties of $\mathrm{Si}_{1-x} \mathrm{Ge}_{x}$ alloys: A Monte Carlo simulation with the Stillinger-Weber potential"' In: Phys. Rev. B 51 (8 1995), pp. 4894-4902.

[205] D. P. Sellan, E. S. Landry, J. E. Turney, A. J. H. McGaughey, and C. H. Amon. "Size effects in molecular dynamics thermal conductivity predictions" In: Phys. Rev. B 81 (21 2010), p. 214305.

[206] P. K. Schelling, S. R. Phillpot, and P. Keblinski. "Comparison of atomic-level simulation methods for computing thermal conductivity" In: Phys. Rev. B 65 (14 2002), p. 144306.

[207] S. Shenogin, A. Bodapati, P. Keblinski, and A. J. H. McGaughey. "Predicting the thermal conductivity of inorganic and polymeric glasses: The role of anharmonicity" In: Journal of Applied Physics 105.3, 034906 (2009), p. 034906.

[208] E. S. Landry, M. I. Hussein, and A. J. H. McGaughey. "Complex superlattice unit cell designs for reduced thermal conductivity" In: Phys. Rev. B 77 (18 2008), p. 184302.

[209] Y. Chen, D. Li, J. R. Lukes, Z. Ni, and M. Chen. "Minimum superlattice thermal conductivity from molecular dynamics" In: Phys. Rev. B 72 (17 2005), p. 174302.

[210] Y. He, D. Donadio, and G. Galli. "Heat transport in amorphous silicon: Interplay between morphology and disorder" In: Applied Physics Letters 98.14, 144101 (2011), p. 144101.

[211] E. S. Landry and A. J. H. McGaughey." "Effect of interfacial species mixing on phonon transport in semiconductor superlattices" In: Phys. Rev. B 79 (7 2009), p. 075316.

[212] J. L. Braun, C. H. Baker, A. Giri, M. Elahi, K. Artyushkova, T. E. Beechem, P. M. Norris, Z. C. Leseman, J. T. Gaskins, and P. E. Hopkins. "Size effects on the thermal conductivity of amorphous silicon thin films" In: Phys. Rev. B 93 (14 2016), p. 140201.

[213] S. King, M. French, J. Bielefeld, and W. Lanford. "Fourier transform infrared spectroscopy investigation of chemical bonding in low-k a-SiC:H thin films" In: Journal of Non-Crystalline Solids 357.15 (2011), pp. $2970-2983$.

[214] T. Jeong, J.-G. Zhu, S. Mao, T. Pan, and Y. J. Tang. "Thermal characterization of SiC amorphous thin films"| In: International Journal of Thermophysics 33.6 (2012), pp. 1000-1012.

[215] S. W. Fong, A. Sood, L. Chen, N. Kumari, M. Asheghi, K. E. Goodson, G. A. Gibson, and H.-S. P. Wong. "Thermal conductivity measurement of amorphous dielectric multilayers for phase-change memory power reduction" In: Journal of Applied Physics $120.1,015103$ (2016).

[216] A. France-Lanord, S. Merabia, T. Albaret, D. Lacroix, and K. Termentzidis. "Thermal properties of amorphous/crystalline silicon superlattices" In: Journal of Physics: Condensed Matter 26.35 (2014), p. 355801.

[217] A. France-Lanord, E. Blandre, T. Albaret, S. Merabia, D. Lacroix, and K. Termentzidis. "Atomistic amorphous/crystalline interface modelling for superlattices and core/shell nanowires" In: Journal of Physics: Condensed Matter 26.5 (2014), p. 055011.

[218] K.-H. Lin and A. Strachan. "Thermal transport in SiGe superlattice thin films and nanowires: Effects of specimen and periodic lengths" In: Phys. Rev. B 87 (11 2013), p. 115302.

[219] M. V. Simkin and G. D. Mahan. "Minimum thermal conductivity of superlattices"| In: Phys. Rev. Lett. 84 (5 2000), pp. 927930. 


\section{Appendix}

A total of 7 published journal articles have been a part of the contents presented in this dissertation. However, during my doctoral study, I have been privileged to be part of 13 first author publications and 9 other publications where I have been a co-author. As all of these publications are focused on the field of nanoscale heat transfer, I list them in chronological order below for the interested readers.

22. A. Giri, J.L. Braun, P.E. Hopkins, "Spectral contributions to thermal boundary conductance across solid, liquid and gas interface: A molecular dynamics simulations", to appear in The Journal of Physical Chemistry $C$.

21. J.L. Braun, A. Giri, J.T. Gaskins, S.W King, M. Sato, T. Fujiseki, H. Fujiwara, P.E. Hopkins, "Breaking Network Connectivity Leads to Ultralow Thermal Conductivities in Fully Dense Amorphous Solids", under review.

20. J. Hua, M. Giovanni, A. Giri, T. Tynell, W. Benjamin, K. Eero, P.E. Hopkins, K. Maarit,"Ultralow thermal conductivity in hybrid $\mathrm{ZnO}$ :nanocellulose superlattice thin films", under review.

19. C. J. Szwejkowski, A. Giri, B. Kaehr, R.J. Warzoha, B.F. Donovan, P.E. Hopkins, "Molecular Tuning of the Vibrational Thermal Transport Mechanisms in Fullerene Derivative Solutions", under review.

18. L. Wang, R. Cheaito, J.L. Braun, A. Giri, P.E. Hopkins, "Thermal conductivity measurements of non-metals via combined time- and frequency-domain thermoreflectance without a metal film transducer", Review of Scientific Instruments 87, 094902 (2016).

17. A. Giri, S.H. Wee, S. Jain, O. Hellwig, P.E. Hopkins, "Influence of chemical ordering on the thermal conductivity and electronic relaxation in FePt thin films in heat assisted magnetic recording applications”, Sci. Rep., 6, 32077 (2016).

16. A. Giri, J.L.Braun, P.E. Hopkins, "Effect of crystalline/amorphous interfaces on thermal transport across confined thin films and superlattices", Journal of Applied Physics, 119, 235305 (2016).

15. J.L. Braun, C.H. Baker, A. Giri, M.Elahi, Artyushkova, K., Beechem, T.E., Norris, P.M., Leseman, Z.C., Gaskins, J.T., Hopkins, P.E., "Size effects on the thermal conductivity of amorphous silicon thin films", Physical Review B, 93, 140201 (2016).

14. A. Giri, J. Niemela, T. Tynell, J. T. Gaskins, B. F. Donovan, M. Karppinen, P. E. Hopkins "Heat-transport mechanisms in molecular building blocks of inorganic/organic multilayers," Physical Review B, 93, 115310 (2016). 
13. A. Giri, P.E. Hopkins, "Analytical model for thermal boundary conductance and equilibrium thermal accommodation coefficient at solid/gas interfaces," The Journal of Chemical Physics, 144, 084705 (2016).

12. A. Giri, J. Niemela, C. J. Szwejkowski, M. Karppinen, P. E. Hopkins, "Reduction in thermal conductivity and tunable heat capacity of inorganic/organic hybrid superlattices," Physical Review B 93, 024201 (2016).

11. A. Giri, P. E. Hopkins, J. G. Wessel, and J. C. Duda, "Kapitza resistance and the thermal conductivity of amorphous superlattices," Journal of Applied Physics 118, 165303 (2015).

10. A. Giri, P. E. Hopkins, "Transient thermal and nonthermal electron and phonon relaxation after short-pulsed laser heating of metals," Journal of Applied Physics 118, 215101 (2015).

9. J.-P. Niemela, A. Giri, P. E. Hopkins, M. Karppinen, "Ultra-low thermal conductivity in $\mathrm{TiO}_{2}$ :C superlattices,"Journal of Materials Chemistry A 3, 11527-11532 (2015).

8. A. Giri, J. T. Gaskins, B. F. Donovan, C. Szwejkowski, R. Warzoha, M. A. Rodriguez, J. Ihlefeld, P. E. Hopkins, "Mechanisms of nonequilibrium electron-phonon coupling and thermal conductance at metal/non-metal interfaces," Journal of Applied Physics 117, 105105 (2015).

7. C. Szwejkowski, N. Creange, K. Sun, A. Giri, B.F. Donovan, C. Constantin, P. E. Hopkins, "Size effects in the thermal conductivity of gallium oxide (beta-Ga2O3) films grown via open-atmosphere annealing of gallium nitride $(\mathrm{GaN})$," Journal of Applied Physics 117, 084308 (2015).

6. A. Giri, J. T. Gaskins, B. M. Foley, R. Cheaito, P. E. Hopkins, "Experimental observation of the temperature dependence of nonequilibrium electron dynamics on electron-phonon coupling in thin gold films," Journal of Applied Physics 117, 044305 (2015).

5. R. Cheaito, J. T. Gaskins, M. E. Caplan, B. F. Donovan, B. M. Foley, A. Giri, J. C. Duda, C. J. Szwejkowski, C. Constantin, H. J. Brown-Shaklee, J. F. Ihlefeld, P. E. Hopkins, "Thermal boundary conductance accumulation and interfacial phonon transmission: measurements and theory" Physical Review B 91, 035423 (2015).

4. A. Giri, P. E. Hopkins, "Spectral analysis of thermal boundary conductance across solid/classical liquid interfaces: a molecular dynamics study" Applied Physics Letters 105, 033106 (2014).

3. T. Tynell, A. Giri, J. T. Gaskins, P. E. Hopkins, P. Mele, K. Miyazaki, M. Karppinen, "Efficiently suppressed thermal conductivity in $\mathrm{ZnO}$ thin films via periodic introduction of organic layers" Journal of Materials Chemistry A 2, 12150-12152 (2014).

2. A. Giri, B. M. Foley, P. E. Hopkins, "Influence of hot electron scattering and electronphonon interactions on thermal boundary conductance at metal/non-metal interfaces" Journal of Heat Transfer 136, 092401 (2014). 
1. M. E. Caplan, A. Giri, P. E. Hopkins, "Analytical model for the effects of wetting on thermal boundary conductance across solid/classical liquid interfaces" The Journal of Chemical Physics 140, 154701 (2014). 